\title{
Family Violence Risk Assessment: An early study of police officers' experiences at the frontline
}

by

\section{Stephanie Christine Grant}

\author{
A thesis \\ submitted to the Victoria University of Wellington \\ in fulfilment of the \\ Requirements for the degree of \\ Master of Arts \\ in Criminology
}

School of Social and Cultural Studies

Victoria University of Wellington

2009 


\section{Abstract}

The key notion of a police organisational culture unique to frontline police officers explains why police services are resilient to reform, as past attempts by police management to change the traditions of the street-cop culture have proved difficult to achieve. The key tenets of the organisational culture of frontline policing see street-level police negotiate and resist aspects of the NZ Police family violence risk assessment model to which they do not subscribe. Frontline officers' resistance is an expression of their attitudes and beliefs about 'real' police work. These beliefs are informed by the occupational subculture of which they are a part. Officers in this study also resist the increasing bureaucratisation of their role and oppose attempts by management to change the informal rules by which they operate. The established autonomy and isolation of frontline policing allows officers discretion in their implementation of the risk assessment model.

This thesis examines the experiences of frontline police officers responsible for implementing family violence risk assessment, in selected areas of the Waikato District. The study employed face to face semi-structured interviews with frontline police officers and sought accounts of their experiences of responding to family violence and their use of the risk assessment tools. The research found that officers recognise and support the improvement of the police response to victims of family violence. Despite this, the introduction of family violence risk assessment in the Waikato District has achieved limited change. This study found considerable evidence of a discrepancy between management policy and operational practice, as the risk assessment model is neither implemented as intended by police management or for which it was designed. This is the result of issues with training and tensions between management directives and established practice at the frontline. These challenges faced by NZ Police are typical of the problems encountered by police organisations implementing similar reforms in other Western jurisdictions. 


\section{Acknowledgements}

This study has been both academically and personally challenging and I am indebted to a number of people for their continued assistance throughout this project. Firstly, I would like to thank my supervisor Dr Michael Rowe for his continued guidance and support throughout the development and completion of this study. Thank you also to the School of Social and Cultural Studies for their assistance.

I would like to thank National Police Headquarters for the assistance to conduct this research. In particular, heartfelt thanks to Inspector Ged Byers. To you I am indebted for your assistance and inspiration, for providing the impetus for this study and continued information and guidance throughout the project. Thank you also to the Research Evaluation Steering Committee and the National Statistics Teams for guidance and information on technical matters. To my Police Liaison Officer Marcus Lynam, thank you for you assistance in coordinating access to police stations and officers throughout the Waikato District.

There are also a number of others who have supported my research. I would sincerely like to thank Mum and Dad, for your continued financial support and encouragement not only over the last 18 months but throughout my 'extended' stay at University. Thank you also to Ben, for listening to my ideas and your ongoing support. Thank you to Jenny and Tracy for answering numerous questions over the last eighteen months. Thank you Jo and Richard for your help and continued enthusiasm for the subject.

Finally, thank you to all those constables and senior officers from NZ Police who gave up time from their busy schedules, often on their days off, to speak to me. Many thanks for sharing your experiences and opinions so freely and honestly. You work at times against incredible odds and I hope this study will improve our understanding of the challenging environment in which you work.

Stephanie 


\section{Contents}

1 Introduction $\quad 1$

1.1 The research 3

$\begin{array}{lll}1.2 & \text { Literature review } & 7\end{array}$

2 Methodology 22

2.1 Research design $\quad 22$

2.2 Ethical issues 28

2.3 A discussion of the methodology 30

3 Police officers' understanding and use of risk assessment 33

3.1 Knowledge of the risk assessment review process 36

3.2 Understanding of the purpose of risk assessment 40

3.3 Perceptions of the risk assessment policy 42

3.4 Experiences of family violence risk assessment 50

3.5 Police officers’ patterns of risk assessment use 53

4 Police officers' experiences and perceptions of risk assessment 58

4.1 Officers' comments on the risk assessment tools 58

4.2 The impact of risk assessment on officers' investigations 68

5 A consideration of the environment in which risk assessment is used 78

6 A discussion of the key findings 104

6.2 Concluding comments 121

$\begin{array}{lll}6.3 & \text { Overall conclusions } & 123\end{array}$

$\begin{array}{lr}\text { References } & 125\end{array}$

Appendix A Information Sheet 136

$\begin{array}{lll}\text { Appendix B Consent Form } & 138\end{array}$

$\begin{array}{llr}\text { Appendix C Interview Schedule } & 139\end{array}$

$\begin{array}{lll}\text { Appendix D Analysis Matrix } & 143\end{array}$

Appendix E A copy of the Family Violence Investigation Report 146 


\section{Chapter 1}

\section{Introduction}

Worldwide, family violence is a serious problem. Many families in New Zealand are affected by family violence and the longer the violence continues the more serious it can become (Dobash \& Dobash, 1979; Websdale, 2000; Radford \& Gill, 2006; Martin et al, 2006; Hoyle, 2008). The true extent of family violence remains largely unknown, as the crime is notoriously underreported and underrepresented in the official statistics (Chrichton-Hill, 2004; Newbold \& Cross, 2008), especially the experiences of those women from ethnic minorities (Chrichton-Hill, 2004). As such, estimates of the prevalence of family violence vary considerably, often in relation to the research methodology utilised (Morris \& Reilly, 2003; Lievore \&Mayhew, 2007). While, it is clear that family violence remains a gendered crime in which the bulk of victimisation is borne by women and their children (Edwards, 1989; Wilson \& Daly, 1993), there is growing evidence to suggest the gender symmetry or asymmetry of family violence between intimate partners (Lievore \& Mayhew, 2007) ${ }^{1}$.

Whilst experience of family violence is widespread, some victims are subjected to extreme levels of violence and control, sadly ended in murder for a small number of victims (Dobash \& Dobash, 1979; Walby \& Allen, 2004). New Zealand Police (NZ Police) in the Waikato District recorded 7,097 family violence occurrences in the 2008 calendar year (New Zealand Police, 2009). Additionally, in the years 2000-2004, NZ Police recorded that 54 women were murdered by their male partner. During the same period three men were

\footnotetext{
${ }^{1}$ In a literature review documenting the scale and nature of family violence in New Zealand, Lievore \& Mayhew (2007) cite research involving New Zealand cohorts which supports the claim that women and men are equally likely to perpetrate violence against an intimate partner. There is international support for this finding also. However, they emphasise that little is known about female perpetrated violence, and caution must be taken in interpreting these findings. It must also be remembered that while prevalence of female violence may be equal to that of male perpetrators, the seriousness and frequency of this violence is unlikely to be equal (Lievore \& Mayhew, 2007).
} 
murdered by their female partner (New Zealand Police, 2008a). It is clear that responding to family violence is a common police task.

Against a background of widespread criticism from the public and scholars alike, since the 1970s Western jurisdictions have made increasing use of the Criminal Justice System as a tool against family violence (Dobash \& Dobash, 1979; Edwards, 1989; Carbonatto, 1998; Newbold \& Cross, 2008). Subsequently, the way that we view and respond to family violence has transformed dramatically in the last twenty five years. In particular, the work of NZ Police and their partner agencies in the community has signalled a new era in the police response to family violence in this country (Carbonatto, 1998, Chrichton-Hill, 2004; Radford \& Gill, 2006; Newbold \& Cross, 2008; Hoyle, 2008).

This period has also seen dramatic change to the social and legislative landscape in which the police service operates, as policing itself has also undergone significant change (Davies \& Thomas, 2003; Flanagan, 2008). Flanagan (2008) writes that the police service has never been so comprehensively scrutinised and reviewed as it is today. The police work load has increased at the same time in which accountability, professionalism and bureaucracy have become the focus of attention for police leaders and scholars alike (Rowe, 2007; Flanagan, 2008). In a climate in which accountability, performance and efficiency are prominent concerns, initiatives which promise to improve the police response to family violence are increasingly sought (Davies \& Thomas, 2003; Laing, 2004; Hoyle, 2008; Perez-Trujillo \& Ross, 2008).

Influenced by growing research in the United States in recent years (Websdale, 2000), police services in the UK (Family Violence Coordination Unit, 2007) Victoria, Australia (Perez-Trujillo \& Ross, 2008), and Canada (Kropp \& Hart, 2004), have developed and implemented family violence risk assessment tools in varying forms (Dutton \& Kropp, 2000; Hilton, 2001; Laing, 2004; Humphreys et al, 2005; Radford \& Gill, 2006; Hoyle, 2008). Risk assessment offers an opportunity for police and their partner agencies to decrease the risk of severe and lethal violence for these victims in particular (Laing, 2004; Felson et al, 2005; Hoyle, 2008; Perez-Trujillo \& Ross, 2008). It is this recent introduction of 
a family violence risk assessment model by NZ Police which forms the focus of this study.

\subsection{The research}

The major objective of this thesis is to examine the experiences of the frontline police officers responsible for implementing the NZ Police risk assessment model six months after its introduction. The research draws upon police officers' accounts of their experiences of responding to family violence and their use of the family violence risk assessment model at the frontline.

\section{Research site}

For policing purposes the Waikato District is divided into three Areas: Hamilton City, Waikato Eastern and Waikato Western. The Waikato District has a population of approximately 315,000 people and services an area of more than 15,000km (New Zealand Police, 2008e). The District has over 660 staff and twenty seven police stations, including the District Headquarters in Hamilton City and three sub-area bases at the Thames, Te Awamutu and Hamilton City police stations (New Zealand Police, 2008c; Personal communication, September 15, 2008).

The constables who participated in the research were selected from the NZ Police General Duties Branch (GDB), which includes beat and patrol constables. NZ Police employs nearly 3,500 beat and patrol officers throughout NZ in the GDB. Officers in this branch are allocated on a 24 hours basis to frontline sectional duties (frontline response to emergency calls in the Incident Response Car (I-Car)) and rotational work in the watchhouse (New Zealand Police, 2008b). GDB constables are also known as frontline police officers, and these terms are used interchangeably throughout the thesis. 


\section{Research objectives}

This study examines frontline police officers' experiences of the NZ Police family violence risk assessment model in selected areas of the Waikato District. The research explores officers' experiences and perceptions of the risk assessment tools and the requirements for implementation of the model. The implications of the context of frontline policing and the characteristics of family violence on the successful implementation of the risk assessment model are considered. The research also examines officers' beliefs about the impact of risk assessment on the family violence investigations they conduct and whether the introduction of risk assessment has changed how family violence is investigated. Theoretically, this thesis argues that a broader consideration of police organisational culture is required to understand the practical realities of frontline policing in relation to family violence.

Based on the research objectives the following research questions were developed:

1. What are the characteristics of frontline policing as experienced by constables in the GDB?

2. What are the characteristics of family violence calls for service as experienced by constables in the GDB?

3. What are frontline officers' understandings of the role of risk assessment in preventing the serious harm or death of family violence victims?

4. What are frontline officers' perceptions of their role in the risk assessment process?

5. Do these perceptions affect how frontline officers implement family violence risk assessments and how is this so?

6. Is there a difference between the family violence risk assessment policy and operational practice?

7. Has the introduction of family violence risk assessment changed how constables in the GDB investigate family violence events? 


\section{The contribution of this research}

The impetus for this study arose from an interest in family violence and recognition of the failure of police to adequately respond to this crime, at the frontline in particular. It was hypothesised that an examination of the environment in which the risk assessment model is implemented would allow not only a better understanding of the challenges frontline officers face using structured risk assessment tools, but also contribute to understanding the failure of police organisations to reform current responses to family violence.

This study provides a first insight into the implementation of family violence risk assessment by frontline police officers in New Zealand. The research contributes to the international body of literature on this subject and begins the study of police risk assessment in New Zealand. This study provides an early consideration of GDB constables' perceptions and experiences of the risk assessment tools six months after its introduction. As such, the research extends the knowledge of how and why frontline officers and police organisations face the challenges they do in policing family violence and the implications of this for the risk assessment process. The findings also contribute to existing examinations of the implications of police organisational culture and the problematic nature of officer discretion on previous attempts to reform the policing of family violence.

\section{The structure of the thesis}

This thesis is divided into seven chapters. Chapter one has introduced the research task and the focus of the study. The following section of this chapter reviews the relevant background literature and introduces the key theoretical concepts to be developed throughout the thesis, upon which the research findings are discussed in subsequent chapters. Chapter two provides a detailed account of the methodological approach which was used to conduct this research and the reasoning for the approach taken. The main methodological concerns are outlined in this chapter also. 
The following three chapters present and discuss the key findings from the research. Chapter three begins the data analysis and examines constables' knowledge and awareness of the risk assessment policy and the requirements of the risk assessment tools. Officers' understanding of the risk assessment process and its objectives are also considered, before a discussion of officers' perceptions of the risk assessment tools is undertaken. The middle section of the chapter addresses officers' patterns of use, finding that the significant majority of officers fail to complete the risk assessments at the scene, as required by the policy. Officers' experiences of risk assessment training form the final section of the chapter.

Building upon the background to officers' implementation of the model presented in the previous chapter, chapter four begins by considering officers' comments on the risk assessment tools, discussing their negative and positive perceptions of the model. The second section of the chapter explores officers' perceptions of the impact of the risk assessment model on the investigation of family violence. The chapter concludes that while officers' believe the introduction of risk assessment has improved the family violence investigations they conduct; this change is neither pervasive nor complete.

Consequently, chapter five seeks to explain the reasons for this finding. The chapter firstly considers the key features of frontline policing and family violence, as identified by frontline police officers. The barriers for implementing the risk assessment model in accordance with the policy are considered. It is found that the context of frontline policing and the unique characteristics of family violence are problematic for officers' use of risk assessment. However, drawing upon the findings about officers' pattern of use discussed in chapter three, it is concluded that officers' failure to adhere to the policy requirements stem from the expression of sub-cultural beliefs about street-level police work, rather than the context of their work as the officers' believe. The chapter concludes that it is the interaction between the characteristics of family violence and the operational context of frontline policing by officers aligned to a distinct police occupational culture which poses an obstacle for reform. 
Chapter six provides the main discussion of the key findings of this study. The chapter draws upon the key literature introduced by the literature review in chapter one and the findings presented throughout the thesis. The discussion draws upon the concept of police organisational culture to consider the implications of officer discretion and the influence of the street-cop culture on reform efforts. The material discussed in this chapter is used to explain the findings in this study. The final section of this chapter addresses the important tangential issues which arise from the key findings and concludes the research.

\subsection{Literature review}

The following section provides a review of the background literature which forms the basis of the main discussion in chapter six. The review begins by discussing the historical criminal justice response to family violence to provide the background from which successive family violence reforms arose from the 1970s onwards. Important developments in the judicial and social landscape of policing in New Zealand are considered to demonstrate the context in which family violence risk assessment has arisen. A description of the NZ Police risk assessment model is also provided. The key notion of a police organisational culture is drawn upon to explain why police services are resilient to reform and past attempts by police management to change the traditions of the street-cop culture have proved difficult to achieve. The key concept of discretion and the tension between divergent organisational cultures within the police are utilised in this discussion to illustrate how and why frontline police officers continue to resist reform initiated by police management. This discussion concludes that while some change has occurred, successful reform is an ongoing challenge faced by police organisations. The key literature drawn upon in this discussion demonstrates that sub-culture beliefs about 'real' police work informed by the organisational subculture of frontline police officers and the discretion afforded to this role, enables street-level police to negotiate and resist reforms to which they do not subscribe. 
Historically, the criminal justice response to victims of family violence has been severely inadequate. Family violence was treated as neither a crime nor a serious social problem, particularly by the police but also indicative of wider social attitudes to interpersonal violence at this time. In most instances, family violence was not met by a law enforcement response at all (Sherman \& Berk, 1984; Edwards, 1989; Lerman, 1992). It is now recognised that prior to the 1970s, police in New Zealand, as in other Western jurisdictions, took a minimalist approach to the treatment of domestic violence (Newbold \& Cross, 2008).

It was the work of women's advocates and feminist academics that first drew attention to the suffering of family violence victims, and the gendered nature of this crime (Sherman \& Berk, 1984; Lerman, 1992; Russell \& Light, 2006; Newbold \& Cross, 2008). Over time, their efforts resulted in official recognition of family violence as a crime warranting criminal justice intervention as they highlighted the role of police as 'gatekeepers' to an official response to this form of violence (Carbonatto, 1998; Gregory \& Lees, 1999; Chrichton-Hill, 2004; Radford \& Gill, 2006; Russell \& Light, 2006).

With much of the criticism levelled at police initiated by the women's movement and the work of feminist scholars, early explanations of the inadequate police response challenged the misogynist culture of police (Chan, 1996; Waddington, 1999; Stephens \& Sinden, 2000; Hyman, 2000; Butler et al, 2003; Bazley, 2007). Social science research illustrated the minimalist stance adopted by police in Western jurisdictions to family disputes (Radford \& Gill, 2006; Newbold \& Cross, 2008). Academics in the United States and Britain began to criticise the historical reluctance to arrest in domestic violence cases, identifying that police had failed to consider domestic violence as 'proper' police work (Dobash \& Dobash, 1979; Edwards, 1989; Newbold \& Cross, 2008) and practised mediation and separation as the favoured course of action (Sherman \& Berk, 1984; Edwards, 1989). By minimising the existence and impact of family violence in this way, the justice system served to collude with perpetrators (Busch \& Robertson, 2000), while at the same time blaming and marginalising the predominately female victims of abuse (Busch \& Robertson, 2000; Newbold \& Cross, 2008). 
In response to growing criticisms, developments in the policing of family violence proliferated, and a major transformation in the official response to this crime can be seen from the 1980s onwards (Carbonatto, 1998; Chrichton-Hill, 2004; Radford \& Gill, 2006; Hoyle, 2008). In the wake of the Minneapolis Experiment ${ }^{2}$, with the promise of an effective tool against family violence at last, the early 1980s saw police across the United States and Britain introduce proarrest and mandatory arrest policies with enthusiasm (Sherman \& Berk, 1984; Hoyle \& Sanders, 2000; Newbold \& Cross, 2008). Consequently, in 1987, NZ Police introduced a pro-arrest policy of its own and this marked the beginning of successive police and judicial reform in this country (Newbold \& Cross, 2008).

Following this impetus for change subsequent years saw the launch of the Hamilton Abuse Intervention Pilot Project (HAIPP) in 1991, which replicated the American Duluth Domestic Abuse Intervention Project in Minnesota, American (Pence, 1983). Following disappointing first results from the project in 1992, the pro-arrest policy was refined and again developed further following subsequent HAIPP reports. As a result of these developments, the policy emphasised a multi-agency approach to domestic violence for the first time in 1993. The following year, a new police form known as the POL400 was introduced for all violent domestic occurrences (Newbold \& Cross, 2008). As outlined later in the report, the POLFVIR (Police Family Violence Investigation Report or FVIR) has since subsumed the POL400 (Personal communication, June 16, 2008).

This period was also characterised by major legislative and policy reform and it was against this background that the Domestic Violence Act 1995 was introduced. Importantly, the Act broadened the definition of family violence to include physical violence, sexual abuse, and psychological abuse, as well as recognised the different relationships in which domestic violence is perpetrated

\footnotetext{
${ }^{2}$ In a field experiment on domestic violence, the Minneapolis Police Department tested three alternate responses to this crime: an arrest, advice, and an order to the suspect to leave for eight hours. Based on results that arrested suspects manifested less violence subsequently, than those offenders ordered to leave by police, the experiment concluded that that arrest was the most effective response to domestic violence (Sherman \& Berk, 1984). The validity of this study has since been questioned. Despite this, pro-arrest and mandatory-arrest policies have been implemented by police worldwide (Newbold \& Cross, 2008).
} 
(Barwick et al, 1996; Busch \& Robertson, 2000; Chrichton-Hill, 2004). This legislation established a concerted commitment to the reduction and prevention of domestic violence through a criminal justice approach in New Zealand (Chrichton-Hill, 2004; Busch \& Robertson, 2000; Newbold \& Cross, 2008). NZ Police has since officially recognised the importance of their role in the response to family violence with the inclusion of this crime as an organisational priority (New Zealand Police, 2008d).

Across Western jurisdictions these important developments have supported the development of the current police approach. The contemporary response to family violence utilises a range of initiatives, with examples including specialist family violence teams and family safety teams staffed by uniformed and nonuniformed experts, pro-arrest policies and the use of risk assessment (Personal communication, September 16, 2008; Flanagan, 2008). Inter-agency collaboration between police and support agencies such as Women's Refuge, Child, Youth and Family (CYF) and other government departments play a key role in the criminal justice response also (Carbonatto, 1998; Gregg, 2007; Newbold \& Cross, 2008).

This study pays particular attention to the employment of risk assessment, which is a recent addition to the arsenal of initiatives utilised by police in the current approach to family violence (Websdale, 2000; Kropp \& Hart, 2004; Humphreys et al, 2005; Perez-Trujillo \& Ross, 2008). With the emergence of research demonstrating that a history of family violence is one of the strongest and most consistent risk factors associated with family violence (Belfrage, 2008; Campbell, 1995), this approach sees greater attention directed towards those victims of sustained and lethal violence (Websdale, 2000; Family Violence Coordination Unit, 2007; Perez-Trujillo \& Ross, 2008). The proliferation of risk assessment instruments in recent years has signalled a move towards a more strategic, scientific and evidence-based approach to family violence than that in the past (Family Violence Coordination Unit, 2007; Radford \& Gill, 2008; Hoyle, 2008). Policing strategies which employ profiling, mapping and analysis have been developed across a spectrum of police work and are not confined to the area of family violence (Rosenbaum, 2006). In fact, structured risk 
assessment tools were first utilised by probationary services in the assessment of offenders (Schneider et al, 1996).

While police officers attending family violence situations have always had a "gut feeling" about the likelihood of further violence, the longer term risks of escalation and murder were not central concerns and decisions about risk were based upon personal judgment (Hoyle \& Sanders, 2000; Hoyle, 2008). In contrast, risk assessment tools are based upon an array of social science research, domestic homicide reviews and practitioner expertise, and most tools draw upon information from a combination of these sources (Websdale, 2000; Hilton et al, 2001; Radford \& Gill, 2008; Hoyle, 2008). Risk assessment and risk management strategies promise to increase victim safety, particularly in lethal situations and ensure better collection and use of evidence through a more accurate and structured approach to investigation (Humphreys et al, 2005; Hoyle, 2008). It is anticipated that risk assessment can make police actions and investigations more consistent, limit prejudicial and discretionary decisionmaking, and guide risk management initiatives to better protect victims (Kropp, 2004). Overall, the growing use of risk assessment is viewed with optimism as criminal justice agencies embrace the opportunity to provide a more efficient and rational response to family violence (Hoyle, 2008).

Risk assessment enables frontline police officers to identify the level of risk a victim is at of future violence or murder using probability calculations (Radford \& Gill, 2006; Hoyle, 2008). Risk scores are then used in conjunction with the risk markers identified by officers, as well as victims' accounts of their abuse (Weisz et al, 2000; Campbell et al, 2003; Laing, 2004). Police then engage in risk management activities to monitor and minimise the risk (Humphreys et al, 2005; Radford \& Gill, 2006; Hoyle, 2008). One influential risk assessment tool is the Danger Assessment Scale (DAS) developed by Jacqueline Campbell (Campbell, 1986; 1995). She developed this assessment for use by practitioners to assess the risk of violence escalating and the potential for family violence homicide. The DAS is based on known risk factors for lethal violence, and involves fifteen 'yes or no' questions, producing a score based on the total number of positive answers (Websdale, 2000; Goodman et al, 2000; Campbell et 
al, 2003; Campbell, 2004b). While further evaluation of this tool is required, there is evidence of some validity of this tool for domestic homicide (Weisz et al, 2000; Roehl \& Guertin, 2000).

While interest in risk assessment and its use as a decision-making aid continues to grow worldwide, there is little research on the validity and accuracy of the instruments used (Schneider et al, 1996; Websdale, 2000; Kropp, 2004; Hoyle, 2008; Perez-Trujillo \& Ross, 2008). In particular, how these tools are implemented by frontline police officers is the subject of little research, and warrants immediate attention (Kropp, 2004; Humphreys et al, 2005; Hoyle, 2008). Above all, little is known about how police officers assess risk in family violence situations, and how situational and environmental factors contribute to the assessment of risk (Perez-Trujillo \& Ross, 2008). Websdale (2000) conducted an important review of family violence risk assessment and highlights that the data is yet to provide a rigorous formula for predicting domestic homicide. As such, different risk assessment tools identify different risk factors, placing different emphasis on these factors (Hoyle, 2008). Regardless of this, Humphreys et al (2005) conducted a noteworthy process evaluation of the implementation of the Metropolitan Police Domestic Violence SPECSS ${ }^{+}$Risk, Identification, Assessment and Management model at any early stage ${ }^{3}$. There are a number of striking similarities between the findings of this study and those of the current research, which will be drawn upon throughout chapters three, four and five.

Following international trends, NZ Police have developed a family violence risk assessment model containing three structured risk assessment instrument tools for use by frontline police officers (a copy of the FVIR is included in Appendix C). The risk assessment tools are contained within the FVIR which was redeveloped and introduced with the risk assessment strategy ${ }^{4}$. The FVIR was

\footnotetext{
${ }^{3}$ This was a preliminary evaluation of the Metropolitan Police domestic violence risk assessment model, tested in two sites in London and two sites in West Yorkshire (Humphreys et al, 2005).

${ }^{4}$ A POL400 is an incident form that all officers are required to complete after attending any incident with family violence overtones. This is a requirement irrespective of whether an offence has been committed. The information from POL 400s is transferred to the Family Violence Database, which stores information from all domestic incidents attended by police. The data
} 
piloted by NZ Police during 2007 and implemented nationwide in April 2008 (Hassan, 2007). Prior to this, family violence risk assessment has been used throughout the Waikato District, since 2004. During this period, GDB constables in the area used an early version of the Risk and Lethality Assessment Worksheet, in conjunction with the POL400 (Personal communication, September 16, 2008).

The NZ Police risk assessment instrument is based upon Campbell's Danger Assessment Scale (Campbell, 1986) and a review of overseas literature on risk assessment and predictors of fatal family violence (Campbell, 1986; 1995; Wilson \& Daly, 1993; Websdale, 2000; Roehl \& Guertin, 2000; Hilton et al, 2001; Abrams et al, 2001). The risk assessment model contains three tools which frontline officers use to determine the risk of future serious harm or murder, which accompanies the established POL400 form. The three risk assessments are incorporated in the pre-formatted booklet which makes up the FVIR and includes the documentation frontline officers require when completing a family violence investigation. The report is divided into four sections containing the relevant documentation for each stage of the family violence investigation: investigation, risk assessment, child safety and support to victims. Instructions about each of these sections are included inside the front and back covers. An aide memoir for investigations is also included at the back of the report. The pages of the FVIR are carbon-copied to provide duplicates of the report for police records and the information of police partner agencies.

The Risk Assessment Questions for Adult Victims (B1) is the first risk assessment tool and involves three questions for officers to ask victims about frequency, seriousness and safety concerns. This is followed by the Red Flags Risk Factors Assessment (B2) in which officers use a list of twelve 'red flags' to predict lethality. The final tool is the Risk and Lethality Assessment Worksheet (B3), and involves a checklist of risk markers of serious and lethal violence. This assessment quantifies risk with a numerical score based on the presence or

collected includes offender/victim histories and past police responses, and so on (Carbonatto, 1998; Newbold \& Cross, 2008). 
absence of risk factors. These scores are used by the relevant District Family Violence Coordinator to monitor and manage at-risk cases.

A formal written policy establishing the requirements and principles of the family violence risk assessment model is currently under development by NZ Police (Personal communication, November 5, 2008). In the interim, officers have been informed of the two main requirements for implementing risk assessments. Firstly, police officers are required to complete all relevant sections of the FVIR, including three risk assessments for all family violence incidents and offences attended. This is a compulsory requirement. Secondly, attending police officers must complete the risk assessments at the scene of the family violence event. This is a compulsory requirement also. Before the end of the shift, the completed FVIR including the risk assessments must be given to the section supervisor for review.

While the new framework for risk assessment in family violence cases forms the topic of this study, this is approached through a framework that relates more widely to issues of police organisations, occupational cultures and strategies for police reform. The following discussion begins by considering the some of the challenges of police reform.

That police organisations face considerable challenges implementing new initiatives and achieving reform at the street-level, is a consistent theme throughout the research (Humphreys et al, 2005; Perez-Trujillo \& Ross, 2008). The evaluation of domestic violence policy in New Zealand and the problems encountered are indicative of those typically faced by police organisations in other Western jurisdictions (Hanmer et al, 1999; Humphreys et al, 2005; Hoyle, 2008; Newbold \& Cross, 2008). This literature finds that regardless of official changes to family violence law and criminal justice policy, the improvement of the frontline police response to family violence is unfinished. Family violence policies are still implemented haphazardly and characterised by a discretionary response (Carbonatto, 1998; Cross, 2006), as policing traditions have proved harder to break than police management anticipated (Chan, 1996; Newbold \& Cross, 2008). 
In the case of risk assessment, from the little research available it can be seen that police continue to face a similar set of problems with the implementation of risk assessment tools (Perez-Trujillo \& Ross, 2008). Common problems encountered by police leaders implementing structured risk tools include a failure to accurately collect the information required, a lack of understanding of the risk assessment tools, and the belief held by street-level police that risk assessment tools detract from their professional skills and judgment (PerezTrujillo \& Ross, 2008; Hoyle, 2008). Perez-Trujillo and Ross (2008) argue that experience from the use of risk assessment in other criminal justice contexts, suggests that valid content of the tools is not enough to ensure the successful implementation and use of risk assessment. Indeed, Humphreys et al (2005) highlight that even if the risk assessment tools themselves are proven to be sound, ongoing evaluation of the use of such tools in operational policing is still required.

The discovery of an organisational sub-culture has enabled social scientists to better investigate and explain the failure of police to satisfactorily respond to family violence (Edwards, 1989). Recent considerations of the failure of police reform emphasise the recalcitrance of police culture as a major obstacle to change across a cross-section of areas (Chan, 1996; Stenning \& Shearing, 2005; Chan, 2007; Bazley, 2007). Influential researchers in the field have utilised the concept of police culture to explain how frontline officers use their discretion to negotiate management policy in their work (Reuss-Ianni, 1983; Chan, 1996; Rowe, 2007) as they continue to informally determine how much frontline policing occurs, often in the face of unambiguous and compulsory policy requirements (Cross, 2006; Rowe, 2007). Police culture is a useful concept in understanding many facets of policing, and is drawn upon by this thesis to explain the difficulties police reformers face (Paoline, 2003).

The concept of police culture originated from ethnographic studies of routine police work which uncovered “a layer of informal occupational norms and values operating under the apparently rigid hierarchical structure of police organisations” (Chan, 1996, p.110). These values and norms are shared by members of the occupational group and are learned through a process of 
'culturalisation' alongside the learning of formal rules and procedures. These norms and values can be unrelated to and even contradict organisational rules and regulations (Waddington, 1999). As such, the conceptualisation of this distinctive organisational culture originated from the 'discovery' that police officers exercise extensive discretion in how they enforce the law (Van Maanen, 1975; Waddington, 1999).

Since its original formation, the concept of organisational culture has received much attention and it is now recognised that the organisational culture of police is much more complex, flexible and multifarious in nature (Reiner, 1992; Waddington, 1999; Chan, 1996). With the discovery that multiple subcultures coexist within the police organisation (Reuss-Ianni, 1983; Chan, 1996; ReussIanni \& Ianni, 2005), the concept of organisational culture has been extended to recognise that it is neither "singular, homogenous nor monolithic", as previously thought (Reiner, 1992, p.109).

Most commonly, the organisational culture referred to is that of the street-level police officer who is responsible for day-to-day policing activities on the streets (Reuss-Ianni, 1983). The features commonly ascribed to this culture include suspicion of others, machismo and racial prejudice, and a readiness to stereotype and cover-up misconduct for colleagues, as well as an orientation toward action and a cynical view of police management and paperwork (Reuss-Ianni, 1983; Reiner, 1992; Chan, 2003). The police culture is also known to emphasise law enforcement or 'real' police work over order-maintenance and service roles (Paoline, 2003).

It is these aspects of police culture related to officers' conceptions of their role that inhibit the reform efforts of police management (Reuss-Ianni, 1983; Barton, 2003; Chan, 2007). In particular, there are certain aspects of police sub-culture that have a fundamental impact on the potential for success of any reform agenda. These are police discretion, solidarity, and autonomy (Barton, 2003); the very aspects which are most commonly attributed to the street-cop culture conceptualised by Reuss-Ianni (1983). Barton (2003) argues that in order to understand why police organisations face great difficulty effecting change, it is 
necessary to first consider the role of police in society. Reiner (1992) insists that an understanding of how police officers see the social world and their role in it, the 'cop culture', is crucial to any analysis of what they do. Barton (2003) contends that the uniqueness of the police role leads to the conclusion that the most significant impediment to change within police organisations is the police occupational sub-culture. He explains that occupational culture is a force of resistance to reform because officer solidarity and autonomy act as powerful impediments to change.

A key factor impeding the improvement of the police response is the ability of frontline police officers to exercise their discretion to avoid and subvert new policies and initiatives (Barton, 2003; Rowe, 2007). In a study of the pro-arrest policy in New Zealand, Cross (2006) identified issues with policy compliance and officer discretion. She found that despite the unequivocal policy requirements, the conditions of street-level policing allow officers to continue to make the final decision regarding arrest. Likewise, Rowe (2007) identifies how junior officers draw upon their discretionary powers to circumvent those policies and procedures which conflict with their conceptions of the role of frontline police. Therefore an examination of police culture is crucial to any understanding of the failure of police reform.

Recently, academics have begun to critically analyse the concept of police organisational culture to argue that the negative values, attitudes and practices traditionally associated with police culture have become a shallow and convenient explanation for all that is wrong with police organisations (Reiner, 1992; Chan, 2003). Chan (1996) argues that “police culture has become a convenient label for a range of negative values, attitudes and practice norms among police officers” (p.110). Others contend that traditional concepts of police organisational culture should be criticised for presenting an oversimplification of police culture (Paoline, 2003). Chan's (2003) study of the socialisation process of police recruits, found that the existence of a homogenous and stable culture within the organisation could not be assumed. She argued that it could no longer be assumed that police recruits are the passive recipients of cultural knowledge 
during the period of socialisation. Instead, she found police recruits to be active and reflective participants in their socialisation.

In contrast with original conceptions, not all features of this culture are negative. Positive features associated with police culture include camaraderie, shared understanding, trust, and reliable support from colleagues (Chan, 2003). Indeed, there is evidence that this culture allows police officers to survive in the unpredictable, dangerous and isolated environment in which they work (Reiner, 1992; Chan, 1996; Waddington, 1999; Chan, 2003; Paoline, 2003).

While the nature and scope of organisational culture continues to be redefined, the problematic nature of officer discretion is a constant source of concern. Police discretion at the frontline and in the police response to family violence specifically, has been greatly criticised (Edwards, 1989; Hoyle, 2008). Discretion is problematic because it allows officers to express the attitudes and values espoused by the culture. Hoyle (2008) believes that many of the problems faced by frontline police officers charged with implementing new initiatives can be explained by a consideration of the consequences of officer discretion.

Given the largely invisible and unsupervised environment of frontline policing and the duties these officers perform, it is unremarkable that frontline policing is characterised by discretionary decision-making (Goldstein, 1979; Goldstein, 1963; Klinger, 1997; Waddington, 1999; Rowe, 2007). In his study of the organisational culture of NZ Police, John Van der Heyden (1997) draws attention to the paradox of discretion in policing: that those who are called on the most often to exercise discretion are those with the least experience and knowledge to draw upon. Reuss-Ianni (1983) explains that this results from an immediate need for action and decision. Indeed, discretion is argued by many to be both an inevitable and desirable feature of frontline police work (Waddington, 1999; Reiner, 2000). It is not the exercise of discretion in itself which is problematic, but the inappropriate expression of discretion (Rowe, 2007).

In the pursuit of organisational reform, the high level of discretion frontline police have and the isolated and unsupervised nature of much of the decisions 
they make allows frontline police to successfully and creatively negotiate and resist the policy directions of police management, with which they feel at odds (Reuss-Ianni, 1983; Reiner, 1992). Indeed, cop culture has developed as a patterned set of understandings which help officers cope with and adjust to the pressures and tensions which confront the police (Reiner, 1992).

The multiple cultures which exist within the police organisational culture are also problematic for reform (Reuss-Ianni. 1983; Chan, 1996; Paoline, 2003). The tension between policy and practice played out within the occupational culture of police was the subject of early research by Elizabeth Reuss-Ianni (1983). Conducting an ethnographic study of the police precinct in the United States, she identified two co-existent but conflicting occupational cultures. These she classified as street-cop culture and management-cop culture. The street-cop culture refers to that culture generally utilised to describe the police organisation. Management culture refers to a second culture operating at headquarters level, described as "bureaucratically and valuationally juxtaposed to the precinct copculture” (Reuss-Ianni \& Ianni, 2005, p.299). More recently, Reiner (1992) and Chan (1997) have supported the existence of multiple cultures within police organisations.

The tension between these two cultures poses particular problems for reform (Chan, 2007). The two cultures are increasingly in conflict and this reinforces the resistance of the street-cop culture to attempts from management to change the environment in which they work. The street-cop culture becomes the primary reference structure as officers prioritise the values and norms from the street-cop culture over the rules and regulations imposed by management-cop culture (Reuss-Ianni \& Ianni, 2005). Specifically, street-level police view management reforms with suspicion and cynicism, and the two cultures clash due to their divergent priorities and philosophies for policing (Reuss-Ianni, 1983; ReussIanni \& Ianni, 2005).

Street-level police officers resist and negotiate those changes and reforms which conflict with their beliefs about their roles as frontline police officers, namely, their law enforcement and crime fighting responsibilities (Reuss-Ianni, 1983). In 
the current study, constables support the broader move to formally assess risk in family violence situations, but they do not support management's ideas about how this should occur. In an expression of the conflict between the street-cop culture and the management-cop culture identified by Reuss-Ianni (1983), officers engage in manoeuvring and adjusting management's policy requirements to suit the reality of their role. As a consequence, intended outcomes of organisational restructuring and procedural reforms often result in unintended consequences (Barton, 2003).

Reforms that fail to accommodate the realities of frontline police work require officers to negotiate the dilemmas and tensions that arise. This see officers draw upon entrenched cultural maxims of police work to adapt and adjust the reforms to fit with the everyday experiences and the realities of their role (Bevir \& Krupicka, 2007). When officers interpret these reforms, Bevir and Krupicka (2007) explain how they are transformed, resisted and domesticated in ways unforeseen by police management. The result is largely unforeseen by police management (Holdaway, 1994; Barton, 2003; Bevir \& Krupicka, 2004).

From a similar perspective, the introduction of family violence risk assessment prompts an occasion for 'sense making' for the police officers who must respond to the change and disruption caused by the new model. Firstly Weick et al (1995) and more recently Chan (2007) have utilised the concept of 'sensemaking' to explain the divergence between policy and practice (Chan, 2007). They propose that in order to understand and apply the changes that reforms require within the operational realities of frontline policing (Reuss-Ianni, 1983), officers engage in a process of 'sense-making' (Weick et al, 2005; Chan, 2007). Weick et al (2005) inform us that sense making in organisations is often triggered by feelings of ambiguity or uncertainty and officers engage in this process to render ambiguous and subjective changes tangible. In doing so, officers draw upon maxims of police culture to make sense of, negotiate and domesticate management policy so that it better reflects their views of 'real' police work and the conditions in which it occurs. Officers use their discretionary powers to facilitate and justify this process (Chan, 1996; Chan, 2007). Weick et al (2005) add that while sense making forms a regular undercurrent of organisational life, interruptions to daily 
activities caused by the introduction of reform, prompt new occasions for sense making.

To summarise, the police response to family violence has seen drastic change in the last three decades. Following legislative and policy changes, the use of risk assessment has gained popularity in the United States, Britain, Canada and Australia. In April 2008, NZ Police followed with the introduction of its own risk assessment instrument. Six months after implementation, this study examines the experiences of a small number of police officers who use the risk assessment tools at the frontline. The following chapter provides the methodological approach taken for this research by outlining the research design of this study, the ethical issues which arose and a discussion of the methodology. 


\section{Chapter 2}

\section{Methodology}

The following chapter provides a detailed description of the methodological approach taken for this research, beginning with a discussion of the research design used and including a consideration of the ethical issues involved.

\subsection{Research design}

In accordance with NZ Police requirements, my research proposal was submitted to the NZ Police Research and Evaluation Steering Committee (RESC) at Police National Headquarters (PNHQ). The research was approved by RESC on 18 July 2008 and included the approval of the Waikato District Commander. A research agreement was then entered into between NZ Police and myself. I was assigned a Police Liaison Officer in Hamilton who facilitated access to police staff and premises, as well as access to other resources and information.

At the conclusion of the research, a draft version of this thesis was reviewed by RESC and subject experts from NZ Police, to ensure the correct use of police terminology and to make certain that research on NZ Police is of a high standard. While this was a necessary and important part of the research agreement, NZ Police have not influenced or shaped any of the findings or analysis presented in this study.

\section{Research design and research tasks}

The study employed a qualitative approach, gathering data from face to face interviews with police constables from the GDB of NZ Police, and a literature review. To improve my knowledge of operational policing, a small amount of observation was undertaken. Primary data was collected from thirty "one-onone” semi-structured interviews conducted with constables during three weeks in September 2009, in designated areas of the Waikato policing District. 
Additionally, interviews with five senior officers were conducted prior to the data collection period. The NZ Police Liaison Officer provided a list of senior officers and Police staff that I would benefit from speaking to. These are experienced persons involved in the risk assessment process. The purpose of these interviews was to gain information on Police management's expectations of constables' use of risk assessment and the policy around risk assessment use, as well as an understanding of the nature of family violence and the development of risk assessment in the Waikato District.

A qualitative approach was utilised to allow the information sought by the research questions to be collected within the time and cost restraints of this Masters thesis. A qualitative approach allowed data to be gathered that captured depth and detail of police constables' experiences and perceptions. A semistructured interview approach allowed the range and depth of data required to satisfy the research objectives to be captured. This approach also ensured that the scope of data collected in the interviews was suitably focussed. Thus, while specific questions were developed for the interview schedule, participants were encouraged to elaborate on these, and this led to a wide range of tangential issues being identified. Such issues often arose from officer's spontaneous narratives about their experiences of family violence. Given the constraints of this research it was more important to understand the depth and detail of officers' experiences and perceptions than to apply a quantitative measure. Due to the small sample size and the desire to avoid misrepresenting the results from a select research site as indicative of GDB constables nationally, quantitative analysis was not undertaken.

In conducting the semi-structured interviews I adopted the approach of the “naive innocent,” described by O’Connell Davidson and Layder (1994, as cited in Rowe, 2007, p.281) as “a socially acceptable 'incompetent' who is there to learn from others”. Following this approach I did not assume the form that the officers' answers would take, but allowed them to provide their responses in their own words, as though speaking to someone with little knowledge of police work (Rowe, 2007). My interviewees responded well to this approach, and I 
found that my position as the 'acceptable incompetent' meant that the officers took greater care to explain their responses.

A literature review of New Zealand and international research was conducted to inform the interview questions and situate this study with the context of current developments in the area. A range of literature was analysed, including academic journals and practitioner reports.

The research also involved a small amount of observation, as I accompanied two police officers one evening. During this shift, I attended four family violence calls for service. This was organised by my Liaison Officer and provided an invaluable opportunity to gain a greater understanding of the nature of frontline policing and the characteristics of responding to both emergency calls and family violence specifically.

\section{Subjects and data involved}

As part of the research agreement entered into between NZ Police and myself, I was provided with a database of police constables currently working in the General Duties Branch in the Waikato Police District. This database was divided into the three police areas within the Waikato District: Hamilton City Area, Waikato Eastern Area and Waikato Western Area. Given the number of family violence occurrences attended by frontline officers in the Waikato District, it was anticipated that all officers selected would have attended a family violence event where they would have been required to complete a FVIR and risk assessments, including those probationary constables recently assigned to the area from the Royal New Zealand Police College (RNZPC). Therefore, both GDB constables and probationary constables were included in the research sample. A little under half of constables serving at the frontline in the Waikato District are probationary constables; these are constables within their first two years of service with NZ Police (Personal communication, September 15, 2008). It was felt that probationary constables would provide an invaluable insight into the challenges experienced by young and inexperienced police officers learning to respond to and investigate family violence. 


\section{Sampling procedures and sample composition}

The sample included in-depth interviews with thirty GDB constables and five senior officers. Figure 1.0 on the following page outlines the sample composition which included male and female police officers, constables and probationary constables, and officers working at rural and urban police stations. The thirty officers interviewed represent ten per cent of the total number of GDB constables employed by NZ Police in the Waikato District. The final sample included officers with a range of experience of working in the GDB, different ages and backgrounds, as well as several very experienced constables with considerable experience of operational policing overseas.

Data collection was conducted at a number of research sites. Hamilton Central Police Station provides the frontline response for the Hamilton City Area, so the sample from Hamilton City was selected from this site alone. In the Waikato Eastern Area, stations at Morrinsville and Matamata were chosen. The stations included in the Waikato Western Area were in Huntly, Te Awamutu and Cambridge. During the site sampling selection, the concern arose that the stations initially chosen as research sites would not accurately reflect the demographic composition of the Waikato District, as the stations initially selected were generally more affluent than other stations in the district. Accordingly, the Huntly and Matamata stations were added to allow a more accurate reflection of the Waikato District, as these areas are typically less affluent, providing a more representative of the District as a whole.

The research sample was provided by NZ Police, and was generated from a database of GDB constables. Initially, the sample was based on the number of male and female, and constables and probationary constables in the District to ensure a representative sample. Due to a small number of difficulties faced, the sampling procedure was adjusted. It was a straightforward process to randomly select participants in the rural areas, because individual interview times could be arranged to accommodate these officers' schedules. This was not the case for participants at the Hamilton Central police station. Early in the data collection phase it was discovered that due to the high work pressures and shift work 
schedules of GDB officers there, the ability to arrange interviews with a crosssection of officers from the five GDB sections was unfeasible within the three weeks allocated for data collection. Ensuring the five sections were represented was important to ensure that different supervisory practices across the sections were captured in officers' responses. Thus, the methodology was amended to include other available officers not initially selected.

Sampling difficulties were also encountered at two of the rural stations. At these stations, only two constables were rostered on for each shift, and it was difficult to access the randomly selected officers. However, the number of officers working on section or in the watchhouse at Hamilton Central Station (17-18) at any given time ensured that availability of potential participants was not an issue. So while the officers interviewed in Waikato Eastern and Waikato Western were randomly selected, participating officers from Hamilton City were selected primarily on the basis of availability.

\section{Sample composition}

The sample can be broken down as follows in the Figure 1.0 below:

\begin{tabular}{|c|c|c|c|c|c|c|c|}
\hline \multicolumn{8}{|c|}{ Figure 1.0 Research Sample $(n=30)$} \\
\hline Area & Station & Constables & $\begin{array}{l}\text { Probationary } \\
\text { Constables }\end{array}$ & Males & Females & $\begin{array}{l}\text { Number of } \\
\text { interviews }\end{array}$ & Total \\
\hline Hamilton & Hamilton & & & & & & \\
\hline \multirow{2}{*}{ City } & Central & 8 & 9 & 14 & 3 & 17 & \\
\hline & & & & & & & $\begin{array}{c}17 \\
(57 \%)\end{array}$ \\
\hline \multirow{4}{*}{$\begin{array}{l}\text { Waikato } \\
\text { Western }\end{array}$} & Te Awamutu & 1 & 1 & 2 & 0 & 2 & \\
\hline & Huntly & 1 & 0 & 1 & 0 & 1 & \\
\hline & Cambridge & 1 & 0 & 1 & 0 & 1 & \\
\hline & & & & & & & $\begin{array}{c}\mathbf{4} \\
(13 \%)\end{array}$ \\
\hline \multirow{3}{*}{$\begin{array}{l}\text { Waikato } \\
\text { Eastern }\end{array}$} & Morrinsville & 4 & 2 & 5 & 1 & 6 & \\
\hline & Matamata & 3 & 0 & 3 & 0 & 3 & \\
\hline & & & & & & & $\begin{array}{c}\mathbf{9} \\
(30 \%)\end{array}$ \\
\hline TOTAL & & $\begin{array}{c}18 \\
(60 \%)\end{array}$ & $\begin{array}{c}12 \\
(40 \%)\end{array}$ & $\begin{array}{c}26 \\
(87 \%)\end{array}$ & $\begin{array}{c}4 \\
(13 \%)\end{array}$ & 30 & $\begin{array}{c}30 \\
(100 \%)\end{array}$ \\
\hline
\end{tabular}


Initially, it had been hoped that five female officers could be interviewed to represent the $16.7 \%$ of female officers who work for NZ Police (Mossman et al, 2008, p.5). However, given difficulties around availability and numbers of female officers working at the research sites, a limited number of four (13\%) female constables were interviewed. Given the sample size, it is unknown whether gender had an impact on the interview responses. While reflecting the views of female police officers in any research on police is important, NZ Police remains a male dominated organisation and this is reflected in the gender composition of the research sample.

\section{Data collection procedures}

An initial introductory email was developed in conjunction with my Police Liaison Officer to inform the participants about the research and the researcher, and to generate interest in the study. The Police Liaison Officer contacted each participant selected and their supervisor by email, to which a copy of the information sheet was attached. This included four additional spare participants randomly selected from each research site.

A three week period was dedicated to data collection. Over this time, set days were allocated to the chosen stations at which interview appointment times were made with interested participants. Interviews were carried out at the research sites on a number of different days, including the weekend, to ensure that a range of officers from each of the five GDB sections in Hamilton City were included in the sample.

With the participant's permission, the interviews were digitally recorded and this was outlined on the consent form. Generally, interview participants were comfortable being tape recorded but three participants declined to have their interview recorded. For these interviews, notes were taken throughout using a pre-formatted interview template. These notes were written up in full upon completion of the interview to ensure all the information was retained. When the field work was complete the interview recordings were transferred into audio files and transcribed in full by the researcher. 


\section{Data management}

Firstly, the tape-recorded interviews were converted into electronic sound files and then transcribed in full into a password protected Word Document. The original recordings were deleted. Computer files with electronic copies of the interview transcripts were created and a hard copy of each transcript was stored securely and confidentially at Victoria University.

\section{Data analysis}

The data was analysed manually using qualitative coding methods to identify and sort for key themes. Prior to data collection, key themes were identified from the literature review and then again from the data analysis after the data collection period. On completion of the transcription process, an initial set of key findings were developed as basic discussion points on which to begin data analysis. The themes collected during these processes were combined to form an analysis matrix to guide the analysis. A copy of this is included as Appendix D.

The data was not analysed by rural or urban stations or by the level of officers' experience with police, as officers' experiences and perceptions of the risk assessment model and the context of its implementation was the focus of this research. Despite some differences in the context of family violence events attended by rural and urban officers, these differences did not have a significant impact on officers' perceptions or experiences of the risk assessment tools. ${ }^{5}$

\subsection{Ethical Issues}

The Victoria University Ethics Committed granted ethical approval for the project on 3 September 2008 (Ethics Approval: No 15834). There are a number of important ethical considerations for research on police organisations. Firstly,

\footnotetext{
${ }^{5}$ The geographical isolation of many family violence incidents attended by police in rural areas is a key difference in the police response to this crime. As a result of geographic isolation, rural officers commonly respond to calls for service alone (as opposed to in pairs in urban areas), back-up support is less available and there will be a significant time delay for support to arrive (Personal communication, June 16 2008).
} 
there is the requirement of informed consent. Informed consent was sought from officers' for the use of data for the purposes of a Masters thesis to be published on completion by Victoria University. Participants were provided with an information sheet prior to the interview taking place. This was distributed via email by the Liaison Officer on my behalf and included a brief description of myself and the research. The Liaison Officer informed the District Commander, Area Commanders and supervisory staff in the GDB that the research was being undertaken and what it involved. The information sheet provided potential participants with a brief description of the focus of the study and the procedures to be followed. Participants were assured that involvement was voluntary, informed of their rights as research participants, and asked to sign a consent form. Officers were given an additional copy of the information to read prior to commencement of the interview and reminded that their participation and any data could be withdrawn at any time during the data collection process.

Confidentiality was an important consideration for this project also. Given the small number of officers interviewed for this research, protecting their anonymity was a key concern. In order to do this, all participants were allocated a confidential code number, to ensure that no identifying details could be found on the interview tapes or transcripts. Identifying information such as age, gender or station was not used in the final thesis. I alone had sole access to the code list which was stored securely and confidentially. A very small number of senior staff were interviewed; as a result no direct quotes from these interviews have been included in the final report to ensure that these individuals cannot be identified inadvertently. This information is referenced as personal communication throughout the thesis.

Furthermore, all interview audio tape recordings, interview transcripts, interview notes and the participant code list were stored securely in a locked filing cabinet at the Victoria University School of Social and Cultural Studies. No material identifying participants in the research or linking comments to individuals was removed from this site. All data will be destroyed securely at Victoria University by myself and my supervisor on 4 August 2009, or once the Masters marking and moderation process is complete. 


\subsection{A discussion of the methodology}

The methodological approach is examined to highlight important issues around limitations of the study as well as strengths of the approach taken. The limitations of the methodology are considered first and include issues around access and my initial interviewing skills, as well as the sample size and composition.

Early in the research, it was anticipated that issues with access could limit the methodology as recruiting and interviewing police officers is a common issue faced by those researching the police (Reuss-Ianni, 1983). Indeed, this research encountered a number of difficulties with access. During the data collection period I found that official approval for the research from PNHQ did not automatically ensure access to interview participants or the trust and acceptance of the frontline officers and their supervisors. The key to gaining a true window into my participants' perceptions and experiences was achieved through the rapport and trust that I was able to establish with the officers. However, trust and rapport was built at varying levels, some within a few minutes of meeting them and with others by spending time with them in the I-Car or in the watchhouse.

As a result, issues of access were negotiated as they arose. In some cases this meant interviewing officers in the morning when work pressures were less demanding and officers were more likely to be available, and in other cases, scheduling interview appointments during the weekend or at the end of officers' shifts. The cooperation and support of either the officer in charge of the station or section sergeant was pivotal for securing interview times with officers and on a few occasions the Liaison Officer addressed the difficulties faced in gaining access to participants on my behalf.

As with any first-time researcher, my initial interviewing skills are a limitation of the methodology. Prior to data collection, a detailed interview schedule was developed and reviewed by experienced researchers to guide my questioning, 
and to ensure the data required was gathered. I was concerned that my inexperience with interviewing could result in the failure to ask key questions or recognising areas to probe further. The interview schedule guided me through the initial interviews until my confidence increased and I could guide the questioning independently. I also explained to interviewees that a follow-up phone call may be required if gaps were found in the data. I was fortunate that my participants were more than willing to explain their work in a free and honest way. I believe that the officers valued this first opportunity to talk about the difficulties they faced with the new reporting requirements and appreciated the chance to voice their opinions. Their willingness to participate allowed me to conduct the interviews with confidence.

This study should be considered an early qualitative benchmark of the current experiences and practices of frontline officers completing family violence risk assessments in particular areas of the Waikato District. This research cannot be said to reflect the experiences and practices of frontline police officers in New Zealand. While the research sample was small, those officers involved in the research can be said to be representative of constables in the Waikato GDB. Thus, it is fair to say that the findings provide an insight into the experiences and opinions of a group of officers from the Waikato District who regularly complete family violence risk assessments as part of their general duties.

The research findings could have been strengthened with the inclusion of additional participants outside of the GDB in the sample. Such as, inter-agency partners Women's Refuge and CYF for example. Additionally, the involvement of section supervisors and police trainers in the study would have contributed data about supervisory practices, the review process and the management-cop culture. However, due to the time and cost restraints of this Masters thesis, widening the scope of the research in this way was unfeasible.

The main strength of the methodology comes from the benefits of the qualitative approach taken. A semi-structured interview format allowed the information required to be gathered within the available data collection period. This approach allowed interviewees to discuss related topics which were of importance to them 
and explain the context of their answers, as well as answer the set questions I had designed. A semi-structured interview approach was practical for interviewing police officers who favoured explaining their answers by drawing on examples from their policing experience. I was able to capture these narratives, while also being able to probe and focus on particular points that I thought were important. These were strengths that a quantitative approach did not offer.

Furthermore, the research strongly benefited from the assistance and support of both PNHQ and my Police Liaison Officer in the Waikato District. I was provided with a great deal assistance researching the topic and obtaining access to research participants who would otherwise have been extremely difficult to access. The research benefited greatly from the guaranteed assistance with sampling, access to participants and general assistance with police information and terminology, which the research agreement ensured.

With the following chapter, the thesis will begin the presentation of the findings from this study. Officers' knowledge and awareness of the risk assessment policy and the requirements of the risk assessment tools are a major focus, as it is shown that officers' knowledge and understanding of the initiative directly inform the patterns of use presented. 


\section{Chapter 3}

\section{Police officers' understanding and use of risk assessment}

This chapter is the first of three chapters which present and discuss the findings of this study. This chapter examines constables' knowledge and awareness of the risk assessment policy and the requirements of the risk assessment tools. Officers' understanding of the risk assessment process and its objectives are also discussed. The chapter then considers officers' perceptions of the risk assessments tools and their patterns of use, to show how the implementation of family violence is connected to officers' knowledge and understanding of the initiative. Officers' experiences and comments on the risk assessment training are also considered. The chapter will begin with a brief outline of officers' general attitudes to the family violence risk assessment model.

A significant majority of the constables in this study are positive about the introduction of risk assessment. They recognise the need to improve the police response to family violence, especially in situations where there is a risk of serious harm or murder:

"I think it's good that they [FVT] get sort of a picture of what's happening instead of what's just happening now, so that we can build a bit of a picture of how best to support them" (14).

Encouragingly, a small number of officers believe that they can already see benefits to their family violence investigations from the use of risk assessment:

"I know that working here we can see the benefit of it. We are attending less of those people where you think "oh no not you again", simply because of all the stuff that's going on at the scenes" (17).

The research shows that, irrespective of their opinions of these aspects of the model, the officers in this study are united in their support for any development 
in the police response to family violence. While frontline officers generally support the use of risk assessment, they do have mixed opinions about the utility and relevance of the risk assessment tools and the risk assessment policy around its use. In particular, officers find issue with some aspects of the content of the tools and the situations in which they are to be used.

Officers were asked to first describe their paperwork requirements for a family violence event. Positively, all officers are aware that a Family Violence Investigation Report, including three risk assessments, is required for all family violence events they attend. All officers are aware that this includes both family violence incidents and offences:

"Basically it's a big book thing, that we have to use for every domestic incident, whether it's a verbal argument or a full on assault, from the most minor domestic up to the very serious" (7).

While knowledge of this aspect of the policy is high, frontline officers are less aware that the risk assessments are to be completed before they leave the scene. In fact, a small group of officers are completely unaware that the risk assessments are designed to be used there:

"Are we supposed to be doing it at the scene?" (10).

"I think technically you're meant to do it at the time, as in at the scene" (30).

Inevitably, the risk assessments are not completed at the scene by these officers:

"I haven't heard of a policy to take it [the FVIR booklet to the scene], and if it is then I guess I'm breaking the policy" (2).

"I sort of thought that it was presented in a manner that we're supposed to take it with us and do it on the job?" (10). 
While other officers are aware of this requirement, their responses indicate that they themselves do not follow the policy and do not believe that their colleagues do either:

"I've never seen anyone take it with them or I've never seen anyone fill it in the car afterwards" (5).

For this second group of officers, uncertainty about the policy cannot explain the divergence between the policy and practice. Instead, these officers give precedence to their ideas about when and where risk assessment should occur over the specifications of the policy. These ideas originate from officers' beliefs about 'real' police work and their role, which are informed by the organisational sub-culture of street-level police. This practice enables frontline officers to satisfy their paperwork requirements in a way that better reflects the realities of frontline policing and their own criteria of how frontline police work occurs (Reuss-Ianni, 1983). These findings show how the culture of street-level policing affects when and where the risk assessment process occurs.

While not a major focus of this study, officers' comments indicate that these patterns of use are sanctioned or at least tolerated by section supervisors. Section supervisors play a pivotal role in frontline officers' behaviour and in particular their implementation of the risk assessment process. Reuss-Ianni (1983) explains how supervisors indicate formally and informally to officers under their command that behaviour which is acceptable and unacceptable on the shift. As such, these staff members play a central role in ensuring how frontline officers enact the risk assessment process (Reuss-Ianni, 1983; Humphreys et al, 2005). This finding highlights the importance of securing the commitment and support of not only frontline officers but their supervisors as well, if policy compliance is to be achieved.

Encouragingly, all officers are aware of, and have used the risk assessment tools. Some officers are more familiar with the risk assessments than others, and this is directly related to how frequently they have been used. How often a GDB constable in the Waikato District uses risk assessment is varied and in this study 
was determined by whether the officer is situated at an urban or rural station. Officers stationed in the Hamilton Central Area consistently respond to a higher volume of family violence events per shift than the stations in rural areas (New Zealand Police, 2009). Since the introduction of risk assessment in April 2008, urban officers have completed a greater number of assessments and are subsequently more familiar with the content of the tools. As a consequence, there is a great disparity between individual officers' knowledge of the risk assessment tools; some officers can vaguely describe the questions involved, whereas others can quote the risk assessments entirely from memory. These officers explain how their in-depth knowledge of the risk assessment questions allows them to complete the risk assessments without needing to carry or refer to the FVIR booklet at the scene:

“Once you've filled out a few POL400s you pretty much know what it's going to ask you back at the station so you can record it anyway in your notebook" (30).

"You sort of visualise how the form unfolds, so as you're at the incident you're thinking through it" (18).

This finding demonstrates that constables negotiate and avoid the requirements of the risk assessment policy in order to implement the tool in a way that reflects their beliefs about how the risk assessment process should operate.

\subsection{Knowledge of the risk assessment review process}

The level of frontline officers' knowledge and understanding of the process that occurs after they have completed the risk assessments and the FVIR is varied. The majority of officers are aware that the FVIRs will be checked at some stage, once they have been forwarded to their supervisor. Most officers are aware that their supervisor will check their reports before the information is passed onto other areas of police. Others however, are unsure of and confused about this process, and a small number of officers are completely unaware that their reports 
are reviewed. The following comments are indicative of officers' level of understanding:

"It comes to the sergeant here. He looks through it, makes sure everything's done and then forwards it off to the FVC and I don't know what happens to it after that" (20).

In the Waikato District, the Family Violence Team (FVT) is a specialist unit within NZ Police responsible for the implementation of family violence policy, initiatives and training within the district. In terms of the risk assessment process, the FVT is responsible for reviewing each FVIR including the risk assessments, collaborating with police partner-agencies and devising and implementing risk management with families (Personal communication, September 16, 2008). The FVT is distinct from the Family Safety Team (FST), who are police investigators working with advocates for adult and child victims to address the needs of a family experiencing family violence. The aim of the FST is to provide a multi-disciplinary response to family violence through assessment, case management and monitoring (Gregg, 2007).

Once the section supervisor has checked the FVIRs, officers know little about who is responsible for checking and monitoring family violence risk assessment. In particular, whether this is the role of the FVT or the FST, and which team liaises weekly with police partner-agencies:

“Once I've completed the report it goes through to our sergeants who forward it off to various agencies, Family Violence Team, Women's Refuge, CYFs, stuff like that. I don't know what else happens” (6).

Approximately half of all the officers interviewed are aware of the role of the FVT in reviewing family violence reports and risk assessments. Most officers have some knowledge of the role of the FVT, and commonly identify the involvement of Women's Refuge and CYF. They are aware of the weekly meetings held with partner agencies to discuss risk, but most do not know that the risk assessments are read out at the meetings and play a crucial role in the 
risk management process. Thus, officers do not have a clear understanding of how the information they collect is used. Overall, most officers have some knowledge of the role of police teams and their partner agencies. However, while they are aware of where the information is going they are not aware of the broader role of risk assessment. This highlights a crucial gap in their knowledge.

When describing the risk assessment review process, it was common for officers to mention having a report returned to them by their sergeant or the FVT. This is how most officers are aware of the FVT and this process is experienced positively for some officers, but negatively for others. Those who experience a returned report in a positive light, recognise the benefits to the quality of their work as well as important implications for accountability. They recognise and support the importance of the auditing process to ensure reports are accurate and complete. They believe that accurate and quality reporting is essential when the risk assessments are read out and discussed at inter-agency meetings:

“Reports are read out at those meetings, so that's motivation for us to do a good job” (12).

"It is helpful because obviously it keeps us on our toes because we've all got to be accountable for our actions...because obviously family violence is serious and we want to be able to keep everything, everyone in line" (13).

For some officers, this process provides an important opportunity for feedback from their supervisor, especially while they are learning to use the assessments. Others believe that their awareness of this process ensures that further effort is made to complete the assessments correctly. These officers understand the connection between the review process and the growing focus on accountability within policing:

“It's like everything in this job, everything is scrutinised and you haven't done a good job then it will be sent back to you” (12). 
"It's good that happens because anything that does slip through the cracks comes back" (30).

In contrast, a small number of officers experience this process negatively. In a classic expression of the tension between the street-cop and management-cop cultures (Reuss-Ianni, 1983), these officers feel that police management are quick to forget the operational realities of frontline policing and object when their discretion is questioned:

"I feel that they're quite often quick to judge our actions at this domestic when they didn't go to it" (4).

Again, these constables perceive the process to be an indictment on their judgment and professionalism as a police officer:

"We've got a lot of pressure from other people in other departments and they've just got to see that it's quite a high pressure job being on the frontline. You're there at the spot; you've got to make the decisions. And it's easy for them to sort of say oh you haven't done that, well at the time there were all these different circumstances going on. There might be one angry guy over there and trying to interview someone over here so it's quite difficult, quite a difficult job” (30).

Crank (1998) argues that criticism of their actions and attempts at reform are "perceived by many officers as a direct threat to their integrity and authority to 'police', which reinforces rather than diminishes the influence of police culture over line officers” (Crank, 1998, as cited in Barton, 2003, p.3). Similarly, these findings demonstrate how closely frontline police officers guard their discretion, as discretion remains central to the role orientation and world view of officers in this study.

Officers are more likely to perceive the review process negatively when a report is returned because of an issue with the course of action taken at the scene, rather than the content of the report. Officers are less affronted when their paperwork 
compared to their actions, is questioned. This may be because officers view paperwork as a task peripheral to 'real' police work and are used to their paperwork being scrutinised in areas of their work other than family violence. Hence, it was common for officers to express the attitude that officer must "cross your 't's and dot your 'i's' on these reports because of the high level of accountability to which frontline police are held. Conversely, other officers see this as a waste of both their time and that of the FVT to send a report back to them to make for minor or unnecessary changes ("to tick a box") which could be done by the FVT. This led some to describe the process as trivial:

"If I completed the whole thing and forgot to tick two boxes that didn't even really apply to what happened, the thing would get sent back to me and I'd have to tick those two boxes myself" (26).

When this occurs, officers are more likely to feel that they were completing FVIRs solely for the purposes of the FVT. This perpetuates the belief that family violence risk assessment is simply additional paperwork that frontline police are required to complete for the purposes of others. Again, there is a link between officers' knowledge and understanding of risk assessment and their support for the "need to get it right". Hence, officers who understand the reasons for reviewing and returning incomplete or incorrect forms are more likely to view this process positively, than those who do not:

"I think that it's a brilliant idea. It [POLFVIR] goes to the right place so that someone can actually follow up and so something gets done about it” (25).

\subsection{Understanding of the purpose of risk assessment}

As with their knowledge of the risk assessment tools, officers' levels of understanding of the purpose of risk assessment are also polarised; ranging from those who clearly understand the reasons for risk assessment, to those who see it as additional paperwork for management's purposes. Very few officers have a 
full and accurate understanding of the purpose and objectives of family violence risk assessment. One experienced constable recognises this in making the following statement:

"The risk assessments are aimed at preventing homicide. Not many officers know this so they don't understand the background of where the risk assessment has come from” (16).

A large number of officers believe that the purpose of risk assessment is to provide information for the police family violence units and for CYF. Apart from this, they know little about the purpose and objectives of risk assessment, or how the information they gather is used:

"I really don't know what they do with it after that [FVIR is completed]. But some of it seems a bit, well from our point of view it seems a bit pointless but obviously they have reasons for putting it there, that's why it's in there" (4).

Generally, the officers are unaware of the role of risk assessment for tracking and managing the risk of serious harm or death. A limited understanding of the broader aims and objectives of the risk assessment process has led a number of constables to believe that risk assessment is another bureaucratic task loaded onto frontline police, unrelated to the objectives of operational policing:

“They go through them and audit them so if we've made glaring mistakes they'll pick them out and send them back to us. So effectively you're almost writing it for their purposes rather than your own” (26).

"I sometimes think that people, different agencies try and put their work onto the police to do it for them which is why we end up with swimming in paperwork because we're doing everyone else's paperwork” (1).

While the purpose of family violence risk assessment is known and understood by a small group of officers, for the majority, their understanding of the purpose 
of risk assessment and the role it plays in preventing serious harm and death is limited. A number of officers believe that they have been made responsible for collecting information other agencies require and do not see a connection between risk assessment and the frontline response. This finding demonstrates that police management have secured limited frontline support for risk assessment, because officers do not understand the fundamental role they play in the risk assessment process:

"Some officers don't get the purpose of some of the very important and key questions and as a result their risk assessments are not completed as accurately as they should be” (16).

The officers who understand the purpose and objectives of risk assessment are more supportive of the development. The following comments are made by officers who clearly understand the rationale for risk assessment and demonstrate that support for management-initiated change is possible when officers are given the knowledge and understanding to support these changes:

"The purpose is to track the escalation of family violence incidents and offenders, to intervene with violent families early to identify risk markers and implement risk management strategies to prevent future serious harm or death” (19).

"I know that it all comes down to the information that we provide them [FVT and partner agencies], because we're the only ones who are there, who really know that's gone on and if we can't provide an honest or detailed account of what's gone on then no one else is going to know” (14).

\subsection{Perceptions of the risk assessment policy}

This chapter now moves to consider the comments officers made about the risk assessment policy. This discussion considers officers' negative and positive perceptions of the policy. Overall, none of the officers interviewed believe that 
the risk assessments are irrelevant for family violence events they attend, but a significant number of officers have concerns about the extent of the family violence documentation required of frontline police. In particular, the applicability of the risk assessment tools for minor incidents and when they are required.

The officers in this study do not dispute the relevance of risk assessment for family violence events when an arrest is made. However, a significant number of officers do question whether it is necessary for all three risk assessments to be completed for every family violence event they attend. These officers do not believe that the risk assessments are relevant for minor verbal incidents:

"It is frustrating that it has to be completed for verbals where a lot of the questions and the risk score aren't necessary. The report is time consuming and it is frustrating having to complete it for minor verbal incidents” (16).

Generally these comments stem from the belief that constables already spend too much time doing paperwork. They believe that spending time completing the risk assessments impinges on their ability to engage in 'real' police work:

"I reckon in the police they need to create systems that are less papergenerating, because I think that there's about 50\%, no $100 \%$ too much paperwork in this job. Because honestly you can go to a job and be there for ten and fifteen minutes and depending on the job it can take an hour, hours and hours of paperwork thereafter" (10).

Officers believe that additional paperwork should not be required for less serious events and find it difficult to gather the information required in these cases. Instead, officers would prefer the discretion to decide when a risk assessment is required:

"They are relevant I suppose. But again, an offence needs to be committed. If an offence has been committed then you can fill in all those 
questions... If it hasn't, as I say if it's two people shouting at each other you can't really fill them in properly. An offence needs to have been committed for you to fill out that form completely and properly. But a lot of incidents we go to no offences have been committed” (30).

Officers believe that the level of the situation should determine whether the risk assessments are necessary. That is, officers believe that the current risk assessments are more relevant for family violence offences where an assault has occurred. They want the paperwork to reflect the differences between incidents and offences and the limits on information available at family violence incidents:

"They can definitely be used when there's been a male assaults female or more serious thing like that. But for the normal, verbal everyday argument that a neighbour rings up and says oh my neighbour's having an argument, and we have to go there and spend half an hour filling in a booklet...really it's a waste of time” (8).

"I think it's better suited to the more moderate/severe end of the scale at the moment and I think that there's a lot of unnecessaries in there. It's great for when there's an arrest or serious continuing family violence, but for the smaller ones that we get called to it doesn't play any bigger role” (13).

Equally, officers in Humphrey et al's (2005) evaluation voiced concerns about the use of risk assessment for domestic violence incidents perceived as minor. While the need for risk assessment for offences was supported, officers were concerned about the level of paperwork for minor situations. These officers also demonstrated less willingness to take full reports for cases they perceived as minor. Flanagan (2008) writes that efforts to reform the police service have caused the organisation to become process bound. While not necessarily negative, he argues that police run the risk of becoming bureaucratic when officers feel bound by process regardless of the circumstances. There is evidence of feeling process bound in some officers' comments. 
Officers' attitudes to the increase in paperwork that family violence risk assessment necessitates are related to the general resistance of frontline police officers to bureaucracy:

“Being sort of honest and frank, I think most people sort of don't really like the forms. They don't like having to fill the forms in. There's two much time spent filling in forms really. You know we spend hours and hours, probably spend more hours sitting in front of a bloody computer than out on the streets because of the amount of work involved to get people to court and to fill in the multitude of paperwork that we have to do" (11).

Most officers continue to refer to the POLFVIR as the POL400, or informally as the POL-four-thousand or the POL-four million (these names refer to officers' jokes about the number of pages in the report). This attitude shows that officers' are generally dismissive of office-based tasks and are resistant to bureaucracy which they do not see as 'real' police work:

"With domestic violence there's a whole lot of other policies and procedures, and everyone in the community seems to want to put more legislation and involve more paperwork, which is a lot harder on the frontline police officers because we can actually spend a lot more time trying to please every person in society than actually getting out there and doing the rest of our job" (1).

Consistent with overseas studies of frontline police work (Chan, 1997; Flanagan, 2008), constables in this study demonstrate resistance to the bureaucratisation of frontline policing. Overall, officers are opposed to risk assessment because it involves more paperwork, require more investigative effort and are more time consuming. These findings show how frontline police become frustrated and disenfranchised when they feel increasing bureaucratic demands translate into less time spent on 'real' police work: 
"It is very involved, even for a very minor domestic it'll still take an experienced cop ten to fifteen minutes to complete it” (26).

"I guess that the only gripe that the officers would have, is having to do one of those for just a silly little verbal thing that's a one off. But that's not the form; it's the policy of having to do that with it" (10).

However, there is a tension between the specifications of the policy and officers' resistance to surrender their discretion and succumb to the increasing bureaucratisation of their role. Successfully implementing a risk assessment strategy which can accurately track the risk of serious harm and death necessitates that GDB constables complete the risk assessments for all family violence events they attend. This policy removes any need for discretion. If officers are to choose when risk assessment is appropriate, the consistent tracking of risk and escalation cannot occur, as recognised by one officer:

"For your minor verbal domestics or for the lower end of the scale it's probably a little bit much for it but I suppose when you're attending the same people all the time you can start seeing a pattern forming. So I suppose it is a good document to have but I think that it could be a bit easier to use” (22).

The most resistance to the bureaucratisation of their role is exercised by older officers, with considerable experience of frontline policing. They believe that the risk assessments are time consuming to complete because of the different sections involved. However, these comments may relate to the general attitudes of frontline officers to a trend towards increasing bureaucratisation and the increase in paperwork created by the FVIR as a whole, rather than the introduction of structured risk assessment tools alone.

The resistance and dislike of paperwork exhibited by police officers is not new (Reuss-Ianni, 1983; Campbell, 2004a; Chan, 2007; Flanagan, 2008). In this study officers' resistance to paperwork does not result from their disinterest in family violence or the accountability of police to victims, but due to their attraction to 
the 'action elements' of policing (Reiner, 1992; Chan, 2007). Indeed, officers in this study resist the increasing bureaucratisation of other aspects of their work, voicing discontent with the level of paperwork required for prosecution files and drink driving offences. Police officers in Janet Chan's (2007) Australian study of organisational socialisation produced comparable findings.

In contrast to the belief that paperwork should be proportionate to the nature of the event, a small number of officers believe the risk assessments are relevant for all family violence events frontline police attend. Officers with this belief understand and support the use of risk assessment to establish a pattern of violence:

"The risk assessments are very applicable to all domestics attended, because history shows that certain indicators lead to serious harm or death. Risk assessment is the best way to identify at-risk cases. Risk assessments provide a good indication of the level of intervention needed for the family after the frontline response. I totally support the theory and research foundation of the use of risk assessment for family violence" (19).

"A lot of the POL400s that we have to do, a lot of the time it's not warranted. But like I say if you don't do it and build up a picture it can escalate and turn to crap later on” (2).

Thus, officers' support for the policy is linked to levels of understanding of the purpose and objectives of risk assessment. Those officers who challenge the relevance of risk assessment for family violence events are less likely to understand the purpose and objectives of the risk assessment strategy:

“The reason why we have to complete a risk assessment isn't clear. It is frustrating as a frontline officer because you seem to go to the same houses for domestics and wonder what the point of all the paperwork is if it isn't doing anything to stop domestic violence. This makes me think 
that maybe this isn't the best way to use the theory on domestic violence" (16).

These officers draw a link between the paperwork required and the importance of accountability. In fact, the importance of accountability is a strong theme in officers' discussions of risk assessment. They spoke of a growing trend in policing for officers to be held accountable for their actions in the field and how this is achieved by their family violence paperwork. They realise that risk assessment can ensure that all cases of family violence are investigated thoroughly and recognise the benefits of documenting that police have taken the correct steps in their response:

"In NZ now I think that its [family violence] becoming quite a big issue and therefore we've got to be seen to be doing the right thing and to make sure that everything we have done is by the protocol and we haven't missed anything or otherwise it'll come back down to us, we'll be the ones losing our jobs basically" (21).

They explain how family violence is an increasingly high profile crime, and that police continue to face scrutiny about their response:

"Just a huge trend towards covering your butt with different forms and as I guess it's from negative publicity that police have, whether it be rightfully so because places have screwed up or from no fault of their own but either way there just seems to be more and more forms getting created that are doing the same job” (1).

For this reason, these officers recognise the introduction of risk assessment as a positive step towards ensuring greater police accountability. They believe that additional and more detailed paperwork is necessary for NZ Police to improve their response to family violence:

"I'm a fan of it because I think that it needs to be detailed" (12). 
"It is helpful because it obviously it keeps us on our toes because we've all got to be accountable for our actions" (3).

Furthermore, officers feel that in the past police evidence gathering and documentation was limited. They describe how the POL400 form neither expected nor required frontline police to collect thorough information. Some officers believe that the POL400 encouraged officers to record nominal information, to avoid using a supplementary sheet:

"What some people used to do, with the old forms it was like two lines you had and then you needed a supplementary sheet and a lot of people would cram it in there, and literally they'd squeeze it all in there. It just kind of encouraged people not to record detail, a lot of people put a lot of bullshit on them, just verbal $1 D$ and didn't mention the black eye and blood pissing out of her nose. And there's just no point generating a file with that rubbish on it, it defeats the purpose. I guess the fact that you've got a whole page encourages you to write a bit more, put a bit more detail down onto it” (9).

A small number of probationary constables, who have not used the POL400, were astonished to learn that risk assessment is a recent development and a onepage form was all that was previously required. These officers now understand why the FVIR was introduced, believing the previous documentation was inadequate for a complex crime such as family violence:

"And I think from what you're saying if that's what the old one was like then I think that this one is probably far better than it was and a move forward from what it used to be like because that one seemed very limited to what information it had” (21). 


\subsection{Experiences of risk assessment training}

While the risk assessment training is not a major focus of this study, officers were asked to discuss the training received and their satisfaction with it. Not all officers in this study have received training on the FVIR and risk assessment tools, prior to or after implementation. These officers discussed their experiences of using the risk assessments in the absence of training. ${ }^{6}$

The officers who have been trained are either happy with the training received or feel that an extensive amount of training is unnecessary to be able to complete the assessments. These officers described attending a family violence training session which ran for several hours, with a large part of the session focussed on the risk assessment tools. One officer mentioned that case studies were discussed to show how risk assessment could have prevented serious harm or death. A number of these officers stated that extensive instruction on the tools was unnecessary. However, these officers may be confident in their ability to complete the assessments because they had been trained and had a clear idea of what was required, and they may have placed more importance on training if they had not received it.

In comparison, at the time of data collection ten officers (33\%) had not received training on the FVIR or the risk assessment tools. These officers have mixed opinions about whether training could contribute to their work. The officers, who received training after the introduction of risk assessment but before this study, were asked about their initial experiences of the risk assessments. Valuable responses were provided which indicate that officers find the FVIR is straightforward to use without training or that in the absence of clear guidance they "winged it" and completed the report by interpreting the requirements. Some officers did not complete the risk assessment aspects of the FVIR during the period before they were trained.

\footnotetext{
${ }^{6}$ GDB constables receive training every five weeks for one day. This is an organisational training cycle and family training is included in this training schedule, which includes all other training programmes. The training schedule for GDB constables is extensive, so ensuring consistent capture of all staff for training is difficult (Personal communication, November 5, 2008).
} 
A key finding from interviews with officers who had recently (within the last four months) left the RNZPC was that they had not received training on either the FVIR specifically or family violence in general while at the RNZPC. This was the case for all recent graduates from the RNZPC in this study. These officers describe the impact on their ability to complete risk assessments, given that they do not yet have the experience to interpret or judge how to do it:

"I certainly came out of college and thought what the hell is this form? And it's the form that we use every shift, probably five times a shift” (7).

These probationary constables found it confusing to use risk assessment without training, especially in the absence of colleagues who had been trained to provide explanations. As a result, officers use their judgment to complete the report based on their interpretation of what is required. They note that "guessing" plays a large part in how their risk assessments are completed. One officer stated that the FVIRs turned up one day and they were expected to "get on with it really." In a similar vein other officers comment that:

"I think that the first time I did one of these I was working by myself and didn't have anyone around to ask, so just made it up as I went” (22).

"One flaw of the police is that you kind of have to learn as you go" (5).

The absence of training is perceived by some officers as a failure of police management to secure their support. Officers believe that police management place little importance on the views of GDB constables and do not value their support for the initiative. Training is an important opportunity to secure buy-in from officers and may go a long way to increasing officers' levels of knowledge and understanding to subsequently increase their support for risk assessment:

“It wasn't sold very well and we can't see the point of it....I don't think it's had buy-in from officers. I don't think that we're that bothered about it, we get on and do what we've always been doing and it's just another form to fill in" (11). 
Given the influential role of supervisors, as role models for GDB constables and enforcers of the policy (Reuss-Ianni, 1983; Humphreys et al, 2005), it is essential that they are trained accordingly and their support for risk assessment is gained also (Humphreys et al, 2005).

In summary, the general consensus among officers is that satisfactory training involves someone going through each risk assessment tool with them, explaining the requirements and the importance of the information collected, and answering questions. A number of officers who were trained later found the training a good opportunity to ask questions and seek clarification. This highlights the impact that the absence of training may have on officers who do not have the same opportunity to ask questions and clarify the risk assessment requirements.

It is clear from officers' comments on all aspects of the risk assessment tools and the risk assessment process that further training is required to clarify and confirm aspects of the risk assessments. Both officers who had been trained and officers who had not, provided a range of suggestions for additional or future training on family violence risk assessment. The key suggestions made include; greater use of case studies, clear explanations of the meaning and purpose of ambiguous questions, more information about the aims of risk assessment and the role of GDB constables in this process. A significant proportion of officers express an interest in how the risk assessments they complete contribute to the wider risk management strategy. An explanation of the reasons for the inclusion of risk assessment in the police approach to family violence is essential. Those probationary constables who have recently left the RNZPC strongly expressed the desire for family violence training while at the RNZPC and that this training include the aims and objectives of family violence risk assessment, especially their role in the process. Indeed, Kropp (2004) argues that those conducting risk assessments should have considerable knowledge of the dynamics of spousal violence.

In the absence of clear guidance and requirements, officers primarily use their discretion and judgment to complete risk assessment. Those officers who complete FVIRs without training, must rely heavily on their experience and 
professional judgment to complete the reports, which is particularly problematic for probationary constables. In the absence of clear training and guidance, frontline officers rely on traditional maxims of policing to do their job. While older and more experienced constables were generally unconcerned by this, placing greater value on their ability to adapt to new reporting requirements with relative ease, it is particularly problematic when this is required of probationary constables, especially recent graduates from the RNZPC. These officers do not have the same cultural and operational experience to be able to make sense of these reforms as their older, more experienced colleagues can (Chan, 2007).

Both follow-up training for continuous improvement and initial training for new constables should involve completing the risk assessments at the scene. This would ensure that officers understand the logic of the policy, as well as the benefits to their investigations. This would also present an opportunity to ensure officers develop good habits and probationary constables adhere to the policy before cultural ideas about 'real' police work can take precedence.

\subsection{Police officers' patterns of risk assessment use}

The following section provides information about when and where frontline officers engage in the risk assessment process. Risk assessments are completed by constables in the Waikato District at three different locations; the scene, the police car and the police station. Seventy three percent (22) of interviewed officers interviewed completed risk assessments at the station, and this predominately occurs during the last hour of the shift. Three percent (1) of officers complete the assessments at the scene and three percent do so in the patrol car. Sixty seven percent of officers complete the risk assessments in a combination of the three locations. To summarise, the majority of officers do not complete risk assessments at the scene, but record the information they need in their notebook, for when they complete the risk assessment at the station:

"I'd just usually write it in my notebook and talk to them at the time" (4). 
Invariably, officers complete the risk assessments at the station, during the final hour of the shift. Thus, a risk assessment can and does get completed up to eight hours after the call for service is attended ${ }^{7}$, as will be discussed this pattern of use has significant implications for the quality of risk assessments produced.

Some officers complete risk assessments at the station so that they can make the required checks on a police computer; for criminal history or existence of protection orders for example. They believe that it is easier to complete the entire report at the station instead, to free up patrol time to respond to calls for service:

"We tend to find that some of the information you need for the form is back here anyway so you need to do a bit of research to get the background of what you're dealing with, information that doesn't come across on the radio" (28).

Officers commonly discuss the implications of completing risk assessments at the station at the end of the shift. Their comments indicate that reports can be completed quickly, when a number of reports are done at one time:

“Often that means that you're filling your forms in after your shifts finished because you'll go to five domestics in a night, you wouldn't have filled in any of the paperwork for any of them and you'll get back here and then you've got five to do all at once" (7).

This pattern of use has particular implications for the information collected. It indicates that officers do not consistently ask the risk assessment questions at the scene and will instead complete the forms using their interpretation of the victim's feelings. This jeopardises the accuracy of the information passed on to the FVT and police partner-agencies:

\footnotetext{
${ }^{7}$ It is a requirement that all Family Violence Investigation Reports are completed before the shift ends. This is to ensure that the information collected and risk score can be entered into the Police National Intelligence Application and available for Police staff to access (Personal communication, June 16, 2008).
} 
"Often in a lot cases the individual officer's brief observations of the situation will end up giving someone a score, or identifying flags or missing flags that haven't been done" (7).

Others explain how the quality of reporting is compromised when reports are completed at the end of the shift, when officers are tired and distracted:

"Especially at the end of the shift when you're tired and want to go home and yeah you've had enough and you have to try to sort of go through them and fill them in" (5).

"They have to be filled in before you go home...And sometimes I guess that probably means that the forms aren't always the best quality, filled in the best they can because you're onto your fifth one thinking I can't even really remember what happened there" (7).

However, it is not necessarily the case that the risk assessments completed at the station are of a poor quality or inaccurate, if officers are asking the necessary questions at the scene and recording the details in their notebook for later use.

The risk assessment policy specifies that frontline officers attending family violence events complete three risk assessments at the scene. Only one officer stated that they always complete risk assessments at the scene. Thirteen percent (4) of officers commented that they would complete the risk assessments at the scene "if you can":

“I wouldn't imagine that I'd ever do one at the scene” (10).

"Often they're not filled out at the scene, often they're filled out back here so some of the questions just aren't asked of the victim...But I don't think they provide a completely accurate representation because they're not always done in consultation with someone and they're often the officer's individual snapshot” (7). 
The officers who complete their risk assessments at the scene do so because they believe in the benefits this brings, both for victims and police, rather than because the policy requires it. They believe that completing the assessments at the scene is more time efficient and they understand the importance of asking victims the risk assessment questions. These officers recognise that an accurate assessment of risk can only be achieved by using the tools at the scene.

For those officers who complete risk assessment in a combination of locations (67\%), they do so based on the context and circumstances of the event. Irrespective of the policy, officers use their discretion to determine at what time the risk assessment is completed:

"If there's a serious assault or something, if a female or male has been assaulted then I'll fill that out then. But just for the everyday domestics where there's no violence or nothing's happened, I'll take all the details down in my notebook and come back to the station and fill it out once we're done" (8).

"If you attend a serious domestic then the report will be carried out at the scene, reports for moderate cases are carried out in the car and reports for minor domestics are completed back at the station at the end of the shift” (15).

While some officers are aware of the policy, they choose to act in conflict with its requirements. Some officers stated that they do not complete risk assessments at the scene and have not seen a colleague do so:

"Well you're supposed to do it at the job but I never do and never have" (25).

Despite their actual patterns of use, a number of officers (93\%) wish that they could complete risk assessments at the scene, but qualify that this is only possible in an 'ideal world'. These comments indicate that while officers are not 
always unwilling to complete reports at the scene, they feel unable to because of the contextual characteristics of frontline policing and family violence:

"In the perfect world we would sit in the car and do it there or maybe take it in and get them because there's some questions you need to ask the victim. But, I mean, I've never seen anyone take it with them or I've never seen anyone fill it in, in the car afterwards" (5).

To summarise the key findings in this chapter, with the exception of a small number of officers, the frontline police officers in this study do not complete family violence risk assessments at the scene. This practice of use results from a limited awareness of the policy and of the aims and objectives of risk assessment. Officers' narratives demonstrate that their perceptions of the relevance of risk assessment for the family violence events they attend is shaped by their levels of knowledge and awareness of the aims and objectives of family violence, as well as the role of frontline police officers in the risk assessment process.

The next two chapters consider the reasons for these patterns of use, concluding that the operational context of frontline policing and the characteristics of family violence overwhelmingly influence officers' decisions about where, when and how the risk assessment process occurs. The focus of chapter four is on officers' experience and perceptions of risk assessment tools and the impact of the model on the family violence investigations they conduct. 


\section{Chapter 4}

\section{Police officers' experiences and perceptions of risk assessment}

This chapter specifically considers police officers' experiences and perceptions of the risk assessment tools. Officers' comments indicate that while certain aspects of the content of the risk assessment tools are problematic for officers, other aspects of the risk assessments assist officers in the investigation of family violence by accommodating the realities of frontline policing and simplifying their reporting requirements. As with the consideration of officers' perceptions of the risk assessment policy in chapter three, this discussion is divided into an examination of the negative and positive components of the risk assessment tools identified by officers. Apart from those difficulties related to context which are discussed in chapter five, officers did not report experiencing any difficulties with the Risk Assessment Questions for Adult Victims. Officers believe that the questions in this assessment are clear, as are the required answers. Those aspects of the Red Flags (B2) and the Lethality Assessment (B3) which drew negative commentary are discussed first.

\subsection{Officers' comments on the risk assessment tools}

A significant number of constables experience difficulty with the risk assessment questions in the Red Flags and the Lethality Assessments. Some officers find certain questions in these assessments confusing due to the lack of clarity around the meaning and parameters of particular questions, the type of answers required and the relevance of certain questions for predicting the escalation of violence:

"It is unclear what some of the questions mean and some can be interpreted in different ways" (16).

In terms of the Red Flags, the meaning of three questions in particular are identified by officers as especially problematic, these are the questions about; 
'access to weapons', 'easy access to the victim' and 'incidents of animal abuse by the offender':

"There's also "does the offender have easy access to the victim and the children" and again that's funny because even if they've got different addresses they've always got easy access, especially in a smaller town. So that's always a bit of a funny one, to red flag someone for that, even if they lived in Hamilton they can still jump in the car" (17).

"There's a couple of questions in there like, "are they in close proximity to weapons" or questions like that, everyone's got a knife in the kitchen you know” (10).

Similarly for the Lethality Assessment, officers explain how the questions are interpreted by different officers in different ways. With this assessment there is a considerable level of confusion around "proximity to weapons" (B22), “reasonably good health” (A13), “diagnosis of mental illness” (A12) and "history of serious depression" (A11). Again, officers are unsure of the scope of these questions. Some officers think that the questions overlap between the tools and do not understand the role of these questions in the assessments. Subsequently, these questions are seen as irrelevant or unnecessary, given the large amount of paperwork already required for family violence:

"In some parts of the lethality worksheet there are duplications and the same questions are asked for both the offender and the victim and it is unclear what the point of this is" (16).

This confusion causes officers to question the validity and contribution of the risk assessment tools. This finding raises concerns, because as Reuss-Ianni (1983) explains, if frontline officers do not know and understand the purpose and contribution of new initiatives, their alternative beliefs will undermine the quality of the risk assessments produced and limit the success of the risk assessment process. These uncertainties mean officers rely on either their 
interpretations of the questions or the guidance of other officers. This finding has implications for the accuracy of information collected, as one officer describes:

"Most of them were quite hard to answer. They were sort of a judgment call, and they weren't very clear and dry what they were asking you. So that was more about, I hate to say guess work but it was more an intuition on what happened. So it's really hard” (23).

It can be seen that in the face of ambiguity and confusion, officers regularly draw on their professional intuition to use the risk assessment tools. A significant number of officers describe this as guesswork. This is the case for constables and probationary constables alike:

“A lot of the time you're just guessing because you don't actually know. So I personally find them quite hard to fill in" (5).

Some officers' are nervous about interpreting or 'guessing' the answers required. Instead of embracing an opportunity to exercise judgement, officers feel uncomfortable when they are forced to make decisions they feel are not always accurate:

"A lot of the time you're guessing, especially ones that you have to circle, you know "do they have access to weapons", "do they have history of mental illness" and all that sort of thing, a lot of the time you're just guessing because you don't actually know” (5).

On account of this, officers interpret and answer particular questions in different ways, relying on their judgment and maxims of professional and competent frontline policing:

"The lethality scores are very subjective and are completed very inconsistently and are based on officer's individual interpretations on how they think they should do it" (16). 
"I guess they're a useful tool to give some indication of where someone's at but I don't think they provide a completely accurate representation because they're not always done in consultation with someone and they're often the officer's individual snapshot that sort of gets filled out on it as well as that particular time" (7).

For probationary constables, interpreting what is required is more difficult. They feel that while they are developing their skills as frontline police officers they are unable to interpret their paperwork requirements confidently. An experienced officer describes this dilemma below:

"The new guys struggle and fumble around it and what should I write in the circumstances, how detailed should it be, what should I put in, what should I leave out, that sort of thing. Or just see some of the new ones sitting there staring at the blank page looking round hoping that someone will come and say oh just write this, this, and this, and put it through” (7).

While it is common for probationary constables to follow the guidance of experienced constables as they adjust to frontline police work (Reuss-Ianni, 1983; Chan, 2003), experienced constables must have an accurate understanding of the risk assessment requirements for this process to be constructive. There is evidence that some officers incorrectly interpret and pass this 'wisdom' on to others:

"It's just the blind leading the blind and making educated guesses. So if I've been doing it wrong then other staff are doing it wrong as well" (26).

This practice is problematic. In an environment where officers rely on their interpretation and intuition to assess risk, officer discretion is the standard position in the completion of risk assessment. While a common aspect of frontline police work, the use of professional intuition and discretion in the risk 
assessment process compromises the accuracy of the information gathered for identifying and predicting risk.

Officers' lack of knowledge and understanding also generate difficulties with the risk assessment questions, as officers do not understand the answers required or the importance of particular questions for the prediction of serious and lethal violence. In turn, a significant number of officers believe that the Red Flags and Lethality Worksheet are restrictive and subjective. Officers explain how these assessments restrict their ability to reflect the true nature of a violent situation in their reports. They believe that the risk assessments demonstrate only the presence or absence of items on the risk assessment, and are unable to reflect the severity and frequency of individual risk markers:

"There are a lot of different levels to that answer and sometimes the answers have a lot of different levels of severity” (13).

Given the complexity of many family violence situations, some officers find it difficult to assess the risk on a numerical basis:

"It's quite hard to judge someone on a numbers basis, on a negative or a positive when people are quite unpredictable” (30).

Others believe the "yes or no" approach taken by the risk assessments is restrictive because it assumes that family violence events are unambiguous situations:

"I think that what we've got to understand is that dealing with domestic incidents isn't always so cut and dry" (11).

"Domestics are not usually black and white cases. Domestics are very polarised and the lethality worksheet does not reflect this” (16).

Alternatively, officers believe that the risk assessments are restrictive because they provide a snapshot of the level of risk at one point in time, and feel that a 
spectrum of severity would more accurately assess risk, by recognising the polarity of family violence situations:

"I'm not sure that they provide the most accurate picture of a situation; they provide a snapshot of that moment in time" (7).

"Typically I find them pretty hard work and the main reason would be, tick boxes don't really apply to a lot of things, a lot of scenarios. Like it's really easy to tick yes or no but it's probably more a spectrum of how things actually are” (26).

Officers are also concerned that the risk assessments produce negative risk scores. They explain how, if the frequency of violence has increased but the intensity of violence has not, the risk assessments do not capture this. Therefore, they feel more space is required for officers' intuition about the situation to be included:

"The risk and lethality assessment worksheet, that's a little bit hit and miss because it relies on an individual assessment and you're relying on cooperation of victims which sometimes isn't forthcoming, so I don't know how accurate they are to be honest” (24).

"I think that some of those questions are too black and white, where you can't put your own thoughts in" (25).

Officers also consider the risk assessments to be subjective. They believe that the level of risk the assessments indicate and the risk score produced, on some occasions reflect the reporting practices of different officers instead of the actual level of risk. Others believe that the way in which the questions are framed leads to an inaccurate prediction of risk:

"They do not at times reflect the true nature of the offender. Quite often I believe they understate the risk that is there due to the way these questions are" (17). 
"There's been a hell of a lot more occasions that the risk should have been higher. So to me these questions are not as good as they could be" (2).

Indeed, officers provide numerous examples of cases where their own evaluation of risk has been greater than the level of risk reflected by a risk assessment:

“When you look at what you've ticked, it doesn't look right at all, doesn't accurately portray the situation. So I don't think the 'yes or no' should be in there actually I think that your observations and more gut feeling and stuff like that" (25).

From officers' comments on the risk assessment tools, the tension between the street-cop and management-cop cultures is evident. While both groups want to improve the police response to victims of family violence, they differ in how this should occur (Reuss-Ianni, 1983). Management believe in the efficacy of a structured and standardised approach to risk assessment based on compliance and consistency. Conversely, frontline police officers support the idea of assessing risk but believe this should be achieved with professional judgment developed through on-the-job experience. They prioritise their own beliefs about how risk assessment should occur and their failure to use the tools at the scene is a clear expression of this.

These findings are important for the future success and development of family violence risk assessment. If frontline police do not believe that the tools accurately assess risk, less effort will be made to complete the assessments thoroughly. This will undermine the risk assessment process which relies on the thorough and consistent collection of information by frontline police.

It was common for officers to identify the extent of paperwork required in the assessment of risk as a negative aspect of the model. That the risk assessments involve too much paperwork was a common criticism voiced by officers: 
"I'm concerned a little bit with the quantity of stuff that you've got to put in it. I can see the reason for it but what would concern me is the fact that guys who are very busy...there's almost 12 pages here to fill in, it's quite complex at points, it asks a lot of questions, it expects you to ask a lot of questions” (20).

These officers clearly demonstrate a resistance to the increasing bureaucratisation of frontline policing. It was common for officers to express frustration with the level of paperwork their role requires, in a general sense. Many feel that increased paperwork continues to take precious time away from frontline policing and pro-active work. Indeed, police officers' resistance to the increasing bureaucratisation of their role is widely documented (Flanagan, 2008). Again, Janet Chan's (2003) research on police organisational culture describes the dislike of paperwork as a typical element of the habitus of street-level policing. She argues that a resistance towards the increased bureaucratisation of frontline policing occurs primarily because frontline officers fail to see paperwork as a function of 'real' police work.

Officers also explained how elements of the risk assessments tools make a positive contribution to their family violence investigations. The aspects of the tools officers spoke of favourably are those which assist the investigation and documentation of family violence. Firstly, the risk markers provided are seen as valuable indicators of risk. A small number of officers overwhelmingly support the introduction of risk assessment. They believe that the risk assessments tools provide the correct indicators for predicting the escalation of violence, and wholly support their introduction at the frontline:

"The questions that they ask are relevant to assessing the sort of risk that the offender poses to the victim” (18).

Frontline officers value and appreciate that the Lethality Worksheet can quantify the risk of serious harm or death into a numerical score which they use to guide their investigations and actions at the scene: 
"It is a good indication to the officer of how serious the situation they are dealing with is and therefore informs the action that should be taken. The lethality worksheet particularly is useful in indicating level of seriousness" (19).

Officers believe that the risk score is a good check and they use it together with their professional estimation of the seriousness of violence and the level of risk present:

"It's relevant and it's quite a good way of getting a measure as to where, it's quite a quick check, it's not an in-depth check but obviously it's just a quick measure of where this person's at in relation to a threat to a victim” (18).

The creation of a self-sufficient reporting and risk assessment document is seen as a positive and valuable development by officers. Unlike other changes to police forms they experience, officers believe that this development recognises and accommodates the time pressures and situational realities of frontline policing, by making their paperwork requirements more efficient and straightforward. Generally, officers believe that the risk assessment tools provide a clear structure to their family violence investigations, as they work chronologically through the pre-formatted forms, following the structure provided. This is an encouraging finding because an important objective of risk assessment is to structure frontline officers' decision-making by establishing a framework to support their commonsense and professional judgment. Officers in the $\operatorname{SPECCS}^{+}$evaluation appreciated introduction of a formal and standardised structure for assessing risk also (Humphreys et al, 2005).

Officers commonly spoke of the problems they previously experienced remembering and locating the additional forms to accompany the POL400. They recognise that the incorporation of the risk assessments within the FVIR is more efficient: 
"Compared to the POL400, it is easier now because all the documents that we need to complete for a family violence report are all included in the POLFVIR booklet, so we do not have to go and find the individual forms that we might need as we had to do before" (16).

"I think that the new forms are better than the old ones. You get a lot more out of them and they don't take you any longer to fill out because by the time that you go and get all the sheets that you need to fill in for one" (9).

Additionally, a number of officers speak highly of the inclusion of reminder notes and guidelines on the risk assessment forms. This demonstrates that officers are reading and using the guidelines that management have provided and increases the likelihood that the reports are completed correctly. Unlike the POL400, officers find it easier to record the information they gather by following the pre-formatted spaces on the forms. The spaces provided signal to officers when more information is expected:

"It captures an awful lot of information and I can see why it's needed" (18).

Indeed, a number of officers believe that the former POL400 restricted their ability to thoroughly recorde evidence:

“I don't think that the POL400 and a supplementary sheet would allow you to capture that information in the same way. I think that you would get if it you had a cop who spent the time getting it" (9).

"We fill in a lot more detail now than when you had three or four lines on the old POL400 and would learn to condense the description of what happened to fit into these three or four lines" (16).

Given that the investigative framework is provided, a significant number of officers describe the risk assessments as easy to complete and straightforward to 
follow. The pre-formatted design sets out the questions required and the way in which it is to be recorded:

"The POLFVIR and the risk assessments are easy and straightforward to complete. It is clear what you need to do and we learn the questions to ask and which details to get” (15).

The inclusion of compulsory fields in the assessment has allowed police management to channel officers' discretion at family violence investigations to ensure that officers capture the specific information they require.

\subsection{The impact of risk assessment on officers' investigations}

As with other police initiatives which seek to improve police responses and administrative practices, family violence risk assessment aims to enhance the investigative behaviour of frontline police officers. The following section examines officers' beliefs of the impact of risk assessment on the investigation of family violence ${ }^{8}$.

Firstly, officers in this study believe that the introduction of risk assessment has changed the questions they ask victims of family violence, because they ask different and more specific questions. By asking specific questions which focus on risk, officers uncover undisclosed histories of abuse as they capture more evidence about a broader range of issues relevant to the relationship, as their scope of concern is broadened from the current event alone:

“We have gone there just for this but we've uncovered a lot more which is quite good and there could be a serious history of abuse then that's quite good. Because we never used to ask that, we just used to ask basic

\footnotetext{
${ }^{8}$ The majority of the constables and probationary constables interviewed had been working for NZ police prior to the implementation of the FVIR in April 2008 and could thus comment on their behaviour before and after the introduction of risk assessment.
} 
questions and we'd just focus on the one time, so given that's a lot broader now” (3).

“The questions help you find out information that wouldn't have otherwise" (12).

To complete the risk assessments, officers find that they need to ask more questions and must actively discuss issues of risk and repeat victimisation with victims using the specific questions provided. As a result, the reports generated contain a greater quality and amount of information:

"We are asking a lot more questions now and subsequently gathering a lot more information about the context of the relationship or the family situation” (19).

"But now with all these questions it's sort of structured so that if you start asking them then it's going to break down the barriers of theirs and you can actually get more out of them. So in that respect it's really good" (3).

Since the focus of their questioning has changed, officers are more aware of the potential for family violence situations to escalate to serious harm and death. As a result, officers are aware of and thinking about family violence differently. As officers consider their risk assessment requirements at the scene, they must think about the presence of risk and the background of the relationship while dealing with the parties involved:

“It makes you think about things more when you're actually out at a job. It definitely makes you think" (8).

"The new forms and the risk assessments have changed our thinking at domestics, we now make a mental note to ask the questions in the risk assessment sections and check the family's history on the computer" (15). 
The risk assessment process has seen frontline officers become more sensitive to issues of risk, as they actively investigate the seriousness and nature of the situation. While they may have done this before, officers believe they are now more conscious of risk:

“They make you think as well, just about what's going on, so you're sort of looking for things behind the scene and not just dealing with what's happened now"(8).

"It makes you think about other things that might trigger things in your mind that perhaps you didn't think about initially previously” (20).

Positively, officers focus their questioning on safety and risk, because they are in the assessment. Subsequently, officers are pleased when they draw out more information from victims, including admissions of undisclosed abuse:

"The questions that you need to ask for the report open up the doors for you with victims and allow you to probe further into the situation and find out a lot more information that the victim wouldn't have disclosed by themselves and you wouldn't have found out otherwise” (15).

The officers have learnt through experience that if they do not think about the risk questions at the scene, then they will not be able to gather the information required:

"Knowing that you have to fill in the report later means that you make sure that you ask the necessary questions at the scene, so that you'll have the information in your notebook to fill in the report later" (15).

"To be honest when you go there, because you know you have to do that paperwork, the questions that you ask and what's going through your head is, you're just about going down from start to finish through your paperwork” (26). 
This finding is contrasted with officers' comments about the influence of paperwork on their behaviour prior to the introduction of risk assessment. Officers' comments indicate that this crucial information was not always gathered in the past, but depended on the experience of the attending officers. Now, the risk assessments act as a valuable aide memoir for all frontline officers, prompting them to consider the history of the relationship and the victim's level of risk:

"The booklet is a prompt of which questions to ask, questions that you probably wouldn't think to ask without the requirement to complete a risk assessment" (15).

They also describe how family violence documentation was previously a secondary concern, and had little influence on their actions at the scene:

"I guess when you go you know that you have to fill out a risk assessment when you're at the job, you did it before I think but now you are just a bit more thinking how much risk is there here?" (9).

Due to the greater awareness of risk and the specific questions officers are asking, frontline police are investigating family violence more thoroughly. Overwhelmingly, they believe that the introduction of risk assessment has improved the amount and quality of evidence they collect. Specifically, officers believe that the risk assessment questions are a good source of information about the nature and background of violent relationships, and they comment that:

"Often you are very surprised and shocked at what the victims tell you when you ask these questions. You find out a lot more than you would otherwise have done. You get a more accurate idea of what is going on in a situation and find out about other related incidents occurring in the relationship that police are not aware of" (15).

“We haven't gone there just for this but we've uncovered a lot more which is quite good and there could be a serious history of abuse" (3). 
The information officers collect is of a higher standard, because it is focussed on the severity of violence and the presence of risk. These officers believe that the risk assessments tools enable them to investigate family violence in a more indepth and probing way because they now ask questions about the relationship and the nature of the violence, instead of investigating the current incident alone:

"It's good because it makes us investigate things a lot more by the questions that we do ask, so it's just not one offence it could be four others that we didn't know about” (3).

"We ask a lot more questions now and we know now which questions to ask to find out more information about the family and what else has been going on" (6).

The responses that officers are able to elicit from victims provide a clearer and more accurate picture of the situation, as the victim's feelings about safety are captured. Officers appreciate the contribution of the risk assessments in this aspect:

"Yeah the victim's view of things because that can be a red flag in itself if they're not quite grasping how serious it is" (9).

“There's a question in there "how often does this happen, how often has he or she hurt you" and we never used to ask that but obviously that could bring up more” (3).

Officers also feel that they are less likely to miss important details such as how the victim is feeling and their injuries, as they place more importance and effort on completing family violence paperwork:

"There has been a change in what we do. Family violence investigations are more in-depth because you need to spend more time gathering evidence. You ask more questions and investigate much deeper” (19).

“In the past we'd just come back here, fill out a POL400. And a lot of stuff was missed, like injuries on victims and what the victim thinks about 
what has happened and those questions. It was just never asked and so for those cases they've made a difference" (7).

Despite the positive improvements to the investigation of family violence identified by officers, the level of change sought by the risk assessment process is incomplete. In contrast to the comments of the majority of officers in this study, a small number do not believe that risk assessment has changed how they investigate family violence. These officers demonstrate a distinct resistance to change:

“There's no difference, it's just a bit of paper. The paperwork has changed but it makes no difference to the way that I deal with an incident...the form has made no difference to me" (11).

They agree that while the format in which information is recorded has changed, how they collect this information remains the same. They believe that risk assessment and the FVIR have formalised, rather than changed, existing practice. Predominately, older constables with considerable experience of responding to family violence hold this opinion. These officers believe that experienced officers investigate family violence thoroughly already:

"So nothing has really changed from that sense to this. It's just how we record it and now they're coming out and saying we know what you're doing before and now we're just telling you that you have to do it. It's basically commonsense written down in rules" (1).

"All these forms, you pretty much ask the questions anyway, they are the stock-standard questions that you'd ask in domestic incidents...the information in this FVIR is what we get anyway" (2).

An analysis of these responses reveals that many officers are unaware that risk assessment has in fact changed their investigative behaviour. So while they are not consciously thinking about the report, issues of risk and escalation do feature in their subconscious decision-making. These officers are sceptical and cynical 
of new initiatives, preferring to support the on-the-job expertise of professional police officers. As Reuss-Ianni (1983) writes, for these officers 'good police work' is characterised by a 'gut level' ability to recognise and respond to a situation, rather than the internalisation and adherence to standardised procedures and rules. In evaluating the SPECSS ${ }^{+}$model, Humphreys et al (2005) also found that while officers appreciated having a formal risk assessment framework, they did not believe that it significantly improved the quality of work produced.

However, while these constables do not believe that risk assessment is useful for their family violence investigations, they do recognise the benefit of risk assessment for younger and less experienced constables. Officers in this study cite experience on-the-job attending family violence situations and life experience as the most important characteristics of officers who respond to family violence well:

"An officer who deals with family violence incidents well has experience of family situations in their own life and the common tensions that can arise in families" (16).

However, these are skills which are learned and developed over time. Officers' statements indicate that family violence is a particularly difficult crime for new and young constables to resolve and that the new paperwork requirements are particularly challenging for these officers:

"I haven't been out of college long but we were still using the old ones when I came out and they're a bit, you were sort of like well what do I put in here? But now it's all there in front of you. It's a lot clearer and you just follow it through methodically" (30).

A number of the older and experienced constables see distinct benefits from risk assessment for probationary constables' investigations and they feel that probationary constables engage in a greater level of investigation with the support of the risk assessment tools: 
"We can argue that it's treating us like "numpties" but there is the new cop who needs to learn” (2).

“Even though half the questions are obvious questions I think it's good to have, especially when you've got some of the younger ones coming out of college and not really aware of what life's about so they probably don't actually think of the questions. I think it's a good tick list for them to ask" (21).

Findings from the SPECSS ${ }^{+}$evaluation also found older officers were less likely to see the benefit of risk assessment for their investigations or had changed their investigative behaviour as a result of the model. Again, officers in this study saw risk assessment as an important guide and aide memoir for young and inexperienced officers, but unnecessary for themselves (Humphreys et al, 2005). The reluctance of older constables to recognise the contribution of risk assessment to their work, demonstrates the difficulties NZ Police face securing commitment and support for new developments from this group. Support for the traditional organisational culture and crime fighting role orientation of frontline police is expressed most strongly by the experienced constables in this study. These officers place greater importance on their discretion and professional abilities as frontline police officers than their less experienced colleagues:

"The older and more experienced officers find the new reporting requirements a lot more painful than the younger and less experienced officers and do not want to do it. For younger and less experienced officers the POLFVIR is a much better tool for them to use when investigating family violence because it is a good guide and aide memoir” (19).

These officers hold entrenched beliefs about the nature of 'real' police work, and the role of frontline police. As a result, they are more likely to resist the bureaucratisation of street-level policing than those officers with limited policing experience: 
“They're probably harder for the older ones, because the new ones just say ok this is the form we fill out go and do it and they do what they're told...It's probably harder for us to get the motivation to fill out the book and ask the questions when five years ago we were just doing a sole one page on it" (14).

Similarly, the importance of discretion is reiterated through officers' comments about the impact of risk assessment. Some officers believe that the FVIR and the risk assessment process erode the discretion they previously held to structure their investigations and reports:

“The book's taken commonsense away from what you'd normally do and it's written it out in black and white. So the things you'd normally do are written there so when you're going to fill out the book things that you knew in the past are now written down" (1).

“In a way it's sort of treating us like we're dumb basically. We've got discretion; we know when something's not right” (2).

This comment illustrates that officers value their discretion and judgment as constables and see the FVIR as an attempt by police management to limit their discretion, as they favour compliance over the use officer judgement in the assessment of risk.

As discussed in chapter three, most officers do not complete risk assessments at the scene. While this indicates a significant discrepancy between policy and practice, it does not necessarily follow that all consequences of this are negative for the risk assessment process. Overall the findings discussed in this chapter indicate that the introduction of family violence risk assessments has positively changed how officers think, investigate and record evidence for family violence events. The chapter examined the particular features of the risk assessment tools frontline officers' support, and found that officers' support those aspects of the assessments which they believe enhance the quality and efficiency of their investigations. That is, officers are positive about the aspects of the risk 
assessments that simplify their paperwork requirements and ensure more efficient and structured reporting.

Despite this, there is evidence that the level of change sought by the risk assessment process is incomplete. The risk assessment model is having a greater impact on some officers more than others, and a small number of officers continue to believe that the introduction of risk assessment has not changed the way that they investigate and report family violence. This limited change is partly the result of officers' failure to complete the risk assessments at the scene. Thus, the incomplete knowledge officers have translates into an incomplete change in behaviour. As such, improving the investigation and reporting of family violence is an ongoing process requiring continuous improvement and training. 


\section{Chapter 5}

\section{A consideration of the environment in which risk assessment is used}

Family violence is consistently described by researchers and practitioners alike, as a complex and devastating crime (Hirschel et al, 1994; Felson et al, 2002; Hoyle, 2008). From the narratives of frontline police officers it is clear that the contextual characteristics common to this distinctive crime, such as the effects of severe and repeated violence on victims, the potential for danger, and the presence of alcohol and young children, complicate the police response (Hirschel et al, 1994; Saunders, 1995; Walton-Moss et al, 2005). Despite recognition of the exceptional nature of family violence, earlier research has failed to consider the impact of these characteristics on the capability of police to adequately respond to family violence. Equally, the way in which the environmental realities of frontline policing and the distinct characteristics of family violence are experienced and interpreted by frontline police officers presents a barrier to successful reform has not received adequate consideration. In response, this chapter proposes that it is the interaction between the environment of frontline policing, the nature of family violence incidents and the key features of police organisational culture that creates challenges for the frontline police officers responsible for the implementation of risk assessment. The chapter begins by examining the key characteristics of frontline policing in New Zealand, considering the effect of these characteristics on the implementation of family violence risk assessment.

In the first instance, constables from the General Duties Branch provide the first line of response from NZ Police to society. They are responsible for the initial response to all calls for service from the public; primarily in the form of 111 emergency calls:

“We don't stop and we go to every single incident, every single job in the first instance, so we're on call all the time for everything” (3). 
Frontline officers continually patrol in Incident Response Vehicles until dispatched by Police Communications to calls for service requiring their attendance (New Zealand Police, 2008d). As the first line of response, the day to day activities of GDB constables occur predominately in the public domain and the public location of their work differentiates the role of GDB officers from that of other police employees. In contrast, other NZ Police employees carry out much of their work in a private setting determined by police, acting in response to incidents that have already occurred. Officers feel that the public location of their work distinguishes frontline policing from other police activities:

"Working on the frontline involves being out on the street and responding to calls" (15).

Indeed, they describe the street location of frontline policing in an elevated and romantic manner as special and mission-oriented:

"It's sort of the sharp edge of the sword if you want to look at it that way. It's really the sharp edge that you're dealing with whatever's happening from second to second on the street, whatever society throws at you” (1).

The emergency and crisis based nature of their work further distinguishes their role. Frontline police spend much time reacting to emergencies (Van der Heyden 1997), and officers identify how GDB constables respond to citizens' calls for service when they need help for a crisis or emergency that only police can provide; either when something is happening or has just happened:

"Things that people would find to be the worst time of their life, that one incident in their life that they will remember for the rest of their life, that's pretty much what we go to. Responding to incidents or crisis in the public where quite often something that has been niggling away for a long period of time has all of a sudden come to a head and...it's at that crisis point that the police usually get called in to help" (1). 
Consequently, it is inevitable that GDB constables are responsible for a wide variety of duties. Their duties vary dramatically, both in the form they take, and how serious they are, from the very minor non-criminal occurrence to the most serious law enforcement concern. Thus, with a potentially infinite mandate, frontline police officers respond to any eventuality from road accidents, processing prisoners and disorderly behaviour, to sudden deaths, robberies and homicides. Public relations, file preparation and paperwork form a major of the frontline officer's role also (Bayley, 2005). Indeed, Hughes (1958) describes how frontline policing involves a "bundle of tasks," which includes an almost infinite set of activities (Hughes, 1958, as cited in Van Maanen, 2005, p.283). In his key analysis of police work, Egon Bittner (2005) defines the police role as "something-that-ought-not-to-be-happening-and-about-which-somebody-hadbetter-do-something-now" (p.31). Officers in this study describe the scope and diversity of their role in similar terms:

“We do everything from old ladies who've had their fences tagged right up to murder scenes” (3).

"You go to anything really, like you're dealing with domestics, disorderly behaviours, fights, firearms jobs, vehicle crashes...knocking on little old ladies door just to make sure they're alright because the neighbours haven't seen them...It's virtually anything” (30).

As officers compare this innumerable array of tasks with the narrow focus of specialist police teams, it was common for them to depict their position as the "Jack of all trades":

"What we do, we have to be Jack-of-all-trades, so know enough about traffic to bluff our way through, know enough about various other things to actually get the ball rolling for investigations, or know what to do at certain scenes, serious assaults and various other things. Whereas, I suppose as you get further up the food chain you start to specialise in certain aspects of the job” (6). 
"Where as other kind of areas just focus on one specific sort of area where they just specialise just in that area, where are we are expected to basically cover everything. So in terms of trades we would be hire a hubby and a specialist unit would be like your plumber or your builder. That would be the difference" (5).

Frontline police work in New Zealand and overseas, is not characterised by crime prevention or law enforcement activities alone, but largely involves responding to calls for service to resolve conflict and restore order (Bittner, 2005; Reuss-Ianni, 1983; Bayley, 2005). Reiner (1992) cautions that views of police work as action-oriented are misleading because they overlook the mundane reality of routine police work, which is commonly tedious, repetitive and messy. Ericson (1982) also contends that police see themselves as crime fighters, but spend little time on this activity. These points are important for explaining officers' perceptions of their work, which in turn contribute to explaining the key features of their organisational culture which have important implications for the risk assessment process.

Although street-level police work may be routine, mundane and service-oriented (Bayley 2005; Van Maanen, 2005), it is undeniable that in responding to emergencies, frontline policing is above all reactive, in which officers respond to calls within rigid time constraints generated by organisational priorities and public demands (Van der Heyden, 1997; Manning, 1992). As such, general duties policing in the Waikato district is characterised by a reactive responsebased model, in which frontline officers respond immediately to emergencies. The officers explain how frontline policing commonly involves "chasing their tails”, as Police Communications continually allocate them to attend waiting jobs. Since the advent of the automated response vehicle, the resultant eventsdriven approach to policing has gained prominence internationally and in New Zealand also. Commonly described as "fire brigade" policing, this style of policing sees officers move from job to job patching up situations as they encounter them (Goldstein, 1979). Police officer, John Van der Heyden (1997) suitably describes this style of policing: 
"I quickly became part of a group of 'professional crime fighters' who rushed from job to job in radio-controlled cars attempting to efficiently clear the backlog of crime and incidents reported before and during our shift. Yes, this was 'fire-brigade’ policing” (Van der Heyden, 1997, p.5).

Accordingly, officers compare their primarily reactive role which occurs "in the moment”, with the planned and historical nature of other police work:

"Compared to frontline policing, other areas of policing involve proactive policing and getting out and speaking to people. This is different to general duties policing which involves responding to calls, dealing with the people and the situation involved and then moving onto the next job" (15).

"You're on the street responding to what happens when it happens as opposed to going on about things on a more planned section. They'll plan how they're going to go about this well in advance as apposed to us, we'll get a job and we'll plan it in the couple of minutes we're driving there" (13).

This system of response naturally leads to a style of policing distinguished from other police work by the limited involvement frontline officers can have at the events they attend. While the GDB is responsible for attending the incident in the first instance and completing the preliminary paperwork, other units are responsible for the future progress of the case. While constables deal with a greater volume of incidents they are involved briefly with these cases:

“They've got more time to carry out enquiries and stuff like that, as we'll be sitting down doing some paperwork and next minute we've got a call, a burglary's on or a robbery or a domestic, anything like that and we've go to jump up and go to that, respond to that as quick as we can” (27).

"The difference between us and other areas is that we tend to deal with stuff very briefly. Like we'll go to a job, deal with it quickly and move to 
the next job because there's always other jobs coming in. Other areas like Inquiries or even CIB they have a lot more time to look in-depth with issues than what we can do responding to calls straightaway" (7).

In illustrating this feature of street-level police work, a number of officers liken their role with Accident and Emergency doctors. They explain how, similar to A\&E doctors, frontline police have a fast and limited involvement with cases as they deal with problems as they arise before moving on quickly to the next emergency:

"General Duties you are the equivalent to an $A \& E$, you respond to something, you may be able to sort it out yourself, or you patch it up basically and pass it on to whatever agency looks after that particular problem" (2).

"Often we'll race to a job, try and deal with it the best way that you can to minimise the situation at the time but that doesn't often fix a lot of the problems that are there, it's just separate people, stop things from occurring at the time, drag people back here, quickly whack out a file and send them off to court" (7).

Officers describe how the time pressures of frontline policing often cause paperwork to accumulate. As a result, reports are completed quickly and information is missed or forgotten. One officer describes the impact on reporting behaviour when this occurs:

"Given that we're that far stretched for numbers and there's that much out there we'll complete it at the end of the shift or whenever you can, it's sort of eat when you can, and do what you can when you can because it's so busy out there" (3).

"Because these days too there's even less and less and time...that's why you don't really sit there and ask them all the questions and write it out, 
you kind of just get through it, write down notes that will jog your memory when you get back" (4).

Such time restraints are problematic for the risk assessment process. Successful use of the risk assessment model necessitates that frontline officers attending family violence events investigate and speak with the parties involved carefully and thoroughly, as well as completing the three risk assessment tools required. As such, there is a conflict between the pro-active approach of risk assessment and the reactive and events-driven nature of the policing environment in which the initiative is implemented.

Manning (1992) describes how police management measure the effectiveness of patrol officers by reviewing reports of patrol activities to assess how patrol officers are handling their workloads. As a result, he explains how officers feel increasing pressure to manage their work in a timely fashion as unattended jobs back-up. Subsequently, in an environment where performance and accountability depend on response times and 'clear-up' rates (Klinger, 1997), officers feel that there is little or no time to complete paperwork at the scene when other calls for service require an immediate police response:

"Me and another mate who was in the I-car, we were the only I-car on and we got a request back saying that some of these weren't up to scratch and we said well two guys taking on that much workload $[7$ family violence events in 6 hours], it's just ridiculous" (3).

Officers in Hamilton City experience the time pressures of frontline policing acutely. Hamilton City constables describe how the high volume of family violence calls they attend significantly increases the amount of paperwork they have to do in their job. Rural officers experience time pressures also, as they often work alone to respond to emergencies over large geographic areas. As such, officers are unwilling to complete risk assessments at the scene when other jobs require attendance and are under pressure to complete multiple assessments before the conclusion of the shift. Officers believe that this practice negatively affects the quality of the risk assessments produced: 
"It's just a timing issue. What used to take five minutes to do a one front page and a supplementary page with circumstances now takes a lot longer, ten or twenty minutes. If you're doing three or four or five of theme to do in a shift then you just don't have the time. That's over an hour just to complete FVIRs. So it's a matter of write in the answers so you can get out of it” (14).

Responding immediately to emergencies and the limits on officers' time at the scene which result from this, see officers prioritise calls for service over paperwork. This practice reinforces the cultural belief that paperwork is less important than other frontline activities, as one officer explains:

"You'd like to sit there and take the time to fill them in but when you hear there's another four priority one jobs sitting in the system, a robbery here, an assault here, another domestic there and you're sitting there filling in a form that you know you can fill in later, you kind of think well someone's got to go and do it, and you fill in the forms when you get back" (7).

However, it should be noted that officers complete paperwork at the scene for crimes other than family violence. ${ }^{9}$ Clearly, officers are unaware of the necessity and advantages of completing the risk assessments at the scene. Alternatively, it is important to qualify that officers may not resist the risk assessments because they involve paperwork in themselves, but because they see the amount of paperwork involved in risk assessment as part of an increasing trend towards the bureaucratisation of frontline policing. They make comments such as, "I didn't join to do paperwork", and they feel that real policing is about action, and paperwork is seen as inaction because it takes them away from the street in which action occurs (Reiner, 1992). As previously highlighted, resistance towards paperwork is a key feature of the street-cop culture to which these officers subscribe.

\footnotetext{
${ }^{9}$ GDB constables routinely complete paperwork at the scene of an incident, such as for traffic offences for example (Personal communication, June 16 2008).
} 
The trend towards increasing paperwork and its accompanying focus on accountability leads some officers in this study to believe that the realities of frontline work are not considered when management develop initiatives implementation by the General Duties Branch:

"I understand where they're coming from, I understand what they're trying to do but I just don't think that it fits with the reality of the job. They just need to kind of redefine it a little bit to suit the frontline police officer a little bit more" (5).

This is a classic example of the street-cop versus management-cop conflict conceptualised by Reuss-Ianni (1983). Officers believe that the bureaucracy utilised by the management culture in their work is not possible within the dangerous, time-pressured, and unpredictable environment of frontline policing. Indeed, while the everyday activities of frontline police officers are mostly routine and unsurprising, the specifics of individual occurrences are largely unpredictable, as police respond to whatever the public calls them to do:

"Society in itself predicts what we have to deal with" (1).

Indeed, Bayley (2005) writes how "patrol work is determined almost entirely by what the public ask the police to do" (p.114). Family violence is an especially unpredictable crime, involving an innumerable set of behaviours that officers must deal with (Barwick et al, 1996; Busch \& Robertson, 2000; Chrichton-Hill, 2004). Typically, the actual circumstances of a family violence event are largely unknown before police arrive at the scene:

“Generally you don't always know what you're getting yourself into" (27).

"Sometimes it can be the nastiest job you may go to, you get abused and stuff like that, or it can be quite mundane, you just never know” (6). 
This unknown and unpredictable environment necessitates that officers are prepared to respond to any situation; from a verbal argument to a potential homicide. Van Maanen (2005) highlights that because police are trained to react to any eventuality; officers view their work as uncertain and ambiguous. Bayley (2005) argues that while patrol work involves mostly minor and non-criminal events, it is nonetheless loaded with uncertainty. He identifies that the dilemma for patrol officers is that they must prepare for war even though they are rarely called upon to fight. Indeed, Bittner (1979) identifies the potential for danger as a defining characteristic of police work. Reiner (1992) similarly argues that police officers are unique because they are required to face situations where the risk to them lies in the unpredictable outcomes of their interactions with the public, rather than the more predictable physical or environmental risks of their job. Thus, one thing which is consistent about frontline policing is its uncertainty (Reuss-Ianni, 1983), as officers in this study recognise:

“That's like any incident you go to actually, the potential to flare up is at just about every job” (25).

No more is this feature of frontline policing evident than in the case of family violence, which involves both a complex set of dynamics and a wide range of dangerous behaviours (Barwick et al, 1996; Busch \& Robertson, 2000; Chrichton-Hill, 2004). In this study, officers comment on the broad nature of family violence which now legally includes a number of different relationships and behaviours of varying severity; from a verbal incident to a violent assault:

"Domestic violence is obviously a wide ranging thing. We deal with calls, with a mother and her ten year old kid and the mother is yelling and the neighbours heard it and thought police should go around there, to someone who's beating the crap out of their partner with a metal bar. So it's quite an extreme thing family violence" (7).

In relation to the assessment of risk in this environment there may be little attending officers can do to mitigate the unknown and unpredictable variables which affect the implementation of risk assessment, officers must nevertheless 
think and act to limit the unknown and the unsafe. Indeed, the inability to manage risk frustrates officers and they spoke of the limited information available from NZ Police Communications and the unreliability of this information:

"Most of the time the information that we receive isn't enough, you don't know a lot of what's going, messages could be garbled. Because some rings the police, it goes through Comms, it goes through to the call taker who then makes a summary of what the person said, that goes into the machine then the dispatcher calls us. So sometimes can be a bit like Chinese whispers" (24).

Ericson (1982) explains how patrol officers are dependent on information from dispatchers because they must trust them and the information they provide. Due to the unreliability of the dispatch process, officers tend to expect the worst, so as to proceed in the safest way (Ericson, 1982). As a result, officers are preoccupied by concerns unrelated to paperwork when they attend family violence situations. They point to innumerable sources of danger at family violence events, including; unknown entry and exit points, the presence and availability of weapons such as knives, tools and firearms, the common existence of drugs, and the presence of alcohol and elevated emotions:

“We're going into a very emotional situation where people have possibly been involved in an assault already. Quite often there can be children in the place, there's the audible factors with people screaming, quite often lights will be smashed out at night so you're going into a darkened environment, you're going into a place where if you're dealing with them in the kitchen, then there's hot stoves, there's hot things on ovens, there's knives all over the place, there's scissors. Basically they could pick up and use anything as a weapon, so officer safety in those sorts of situations is obviously paramount" (1). 
Understandably, safety is critical to officers responding to family violence. While some constables in particular spoke of other colleagues being seriously injured, all officers are keenly aware of the dangers of family violence for police:

"Yeah domestics can be extremely dangerous for the police, because we are going into basically the domain of the people we are going to deal with, so basically it's their safe haven, it's their strong hold” (1).

“Typically there's always going to be safety concerns and issues going to a domestic as apposed to some of the other jobs you go to where things have already happened" (26).

Concerns about safety are heightened for rural officers in this study, who regularly attend family violence events alone and often at night. For rural officers, their concerns about safety are intensified by the isolation and unavailability of back-up:

"You're on your own in this situation and you think "**** how much pepper spray have I got in here"? There's not two of you out, you're on your own and they [offenders] know that you're on your own, they know as well as you do how many are on in **** and that the nearest cop will be in $* * * *$ or $* * * * ”(10)$.

The imminence of danger ensures that concern for officer safety is paramount and officers are uncomfortable completing the risk assessments at the scene. Officers express great resistance to using the FVIR booklet at the scene. They feel vulnerable holding the booklet at the scene, and prefer to keep their hands free. Consistent with comments made by Bayley (2005), whether or not officers are in danger, they need to be prepared for it. Thus, they believe that carrying a FVIR booklet inhibits their ability to protect themselves and that this policy requirement fails to recognise the realities of responding to family violence:

“You want you're hands free in case you need to deal with something quickly. Because you can go to what seems like a very minor incident that 
suddenly becomes more serious, so you don't know what you're going to be walking into...some of the guys here have been stabbed and stuff so you don't want to be carrying a form in your hand" (28).

Again, in prioritising officer safety, officers will make decisions about arrest in the first instance, and consider the risk assessment requirements later:

"The paperwork for family violence incidents is very daunting especially in very tense and emotional situations, which is often the case for family violence, where you need to calm the situation down first before even thinking about collecting information for the report" (19).

Building upon officers' patterns of risk assessment use, this chapter has considered officers' descriptions of the ways in which the distinctive characteristics of family violence pose challenges for the implementation of risk assessment in the context of the frontline response. However, the research found evidence that the decision not to complete the report at the scene is made prior to instructions from Police Communications to attend a family violence event, rather than with an assessment of the situation upon arrival as officers suggest. While officers give numerous reasons why they should not complete risk assessments at the scene, from the comments they made about their patterns of use in chapter three, it is likely that they have little or no experience of completing the risk assessments at the scene. Therefore, officers' resistance to carrying and completing reports at the scene requires an alternative explanation.

The research found that officers' patterns of use are linked to their beliefs about 'real' police work rather than direct experience of these situational difficulties commonly highlighted. In their reluctance or refusal to follow the policy, officers express the belief that responding to emergencies is 'real' police work and thereby devalue peripheral tasks like paperwork. These beliefs further serve to reinforce and maintain current practice as officers do not carry reports with them or store them in the patrol car, making it less likely that they will engage in risk assessment at the scene: 
"I've only used it once directly in a house, as in I filled it in at the scene. I forget to take them or I don't have any" (11).

In this way, the dangers inherent in their role mean officers do not complete risk assessments at the scene. While they may not have experience of being in danger from doing so, from their experience as a frontline officer and of responding to family violence, they assess the risks of completing paperwork at the scene and prioritise their judgment over the requirements of the policy. In the same way, the features of family violence and the unique dynamics of this crime are described by officers as a further barrier for completing risk assessment at the scene.

Undoubtedly, family violence is a heartbreaking and personally confronting crime for officers to deal with. Above all, the presence of young children makes it difficult for officers to manage these situations. This is so, both in terms of resolving the situation and for their emotional resilience as well.

"It can be hard to deal with sometimes, especially if there are kids, that's really hard. You know you can turn up to some places and you see mum and dad going to each other and there's a couple of kids watching them you know and crying” (5).

Officers describe how entering a citizen's home can increase the emotional level of the situation if the parties react negatively to police attendance. This in turn can intensify offender's aggressive and territorial behaviours:

“For one thing you're going into someone else's domain. People are very protective of their own surroundings and they dominate their own surroundings, and you're going in there basically as an outsider” (20).

For a number of important and complex reasons, victims of family violence are often reluctant to cooperate with police questioning (Felson et al. 2002). This is often a consequence of the unique and powerful way that family violence offenders control their victims. In these cases, officers speak of the elevated 
emotions and tension between parties, which can be transferred to officers on their arrival, commonly when a neighbour has heard the dispute and called the police. In these cases, the parties involved are unlikely to afford officers the cooperation needed to complete the assessments:

"Sometimes as well the victim can be as anti-police as the offender, even if they've made the call. I mean they call us and then they're like "F-off pigs” (9).

“At other times as well they just don't want the cops there either. So it can be slim to none the likelihood that we can ask them these questions and record what they say" (1).

General hostility and "resistance to the blue uniform" was a common phrase used by officers to describe family violence. When this factor is present, officers find it very difficult to assess risk because they cannot access the information they require:

"A lot of the time they're not interested. Because a lot of the time, although you've got the offender saying "what the hell are you doing here" well sometimes the victim's exactly the same because they might not have called, it could have been a neighbour that called and we turned up completely unexpected in the middle of an argument" (18).

The complex nature of family violence presents a number of challenges for the use of risk assessment at the frontline. Frontline officers consistently emphasise the intense dynamics at play when victims are subjected to sustained abuse and are at risk of serious violence but remain in the relationship:

"With domestics there's a lot that happens behind the scenes that we don't know about, a lot of history, a lot that's happened in between that we don't even know about. So we try and come in and help with one little thing but there's a whole lot bigger going on in the background" (14). 
“There's a lot of emotions flying around, people will look at you and think what are you doing here, you're just interfering in something that's a private matter" (18).

The emotions and stresses involved in family violence complicate officers' efforts to collect evidence and discuss risk with victims. Officers' believe that their attempts to ask the risk assessment questions can amplify already volatile situations. Consequently, officers favour calming the situation down over asking the risk assessment questions, and again paperwork is a peripheral concern:

"It's distressing to see a woman like that, that you've got to try and get her to regurgitate all this information and the more you go on about it the worst it gets for her" (12).

"There's a lot of stuff on there and questions that sometimes at the domestic you haven't got that time [to do paperwork], especially if you've got a volatile situation and you need to take someone out” (20).

This practice can also be seen as an expression of the traditional law enforcement orientation of street-cops (Reuss-Ianni, 1983), as officers continue to favour the action-oriented elements of their work over paper-based tasks:

"A lot of times if it's been a major incident you are taking someone away or you're definitely moving them from the house in some form...so it's not appropriate to pull it out and start filling it out in front of the victims” (2).

The personal and sensitive nature of particular questions about the relationship and the abuse are experienced by some victims as harsh, invasive and irrelevant. In terms of the Risk Factors and the Lethality Worksheet, a number of officers find particular questions difficult to ask victims:

"Some of them can be hard to ask in the heat of the moment, because a lot of them are quite sensitive questions and a lot of them are weird 
questions, like asking someone if their husband's threatened to commit suicide or they harm animals" (13).

Some officers are uncomfortable with the questions because they feel awkward intruding into the private life of others, and some officers will not ask particular questions if they believe victims will react negatively:

"I haven't asked too many but you're sort of digging into their personal life, sometimes they are willing to answer, sometimes they aren't. Sometimes they'll get quite personal, they're quite sensitive, so I suppose it depends on the situation and the people that are involved and their willingness to tell us" (28).

"Some of them are hard to ask because they don't really want to give you that information, especially if it's just an argument and they haven't had the police at their house before and we're intruding into their personal life and trying to find out all this deep and meaningful stuff about them" (14).

As a result, a significant number of officers believe that it is inappropriate to complete family violence risk assessments at the scene, mostly because it is not always suitable to ask the victim the risk assessment questions. This belief is held in relation to all three risk assessment tools and is based on the premise that care must be taken to ask sensitive and personal questions and the scene of a crime is unsuitable for this task:

“Sometimes the victims' questions aren't appropriate to ask at the time, especially in front of the offender for example or children. Sometimes the victim is not in the right frame of mind either" (9).

"The scene can be an inappropriate environment to ask the questions and get the information we need, especially if the offender is present. The victim needs to feel calm and safe in the environment so that they feel able to answer the questions we need to ask" (16). 
Officers with this belief continue to complete the risk assessments at the police station. As a result, some questions are not asked because officers' contact with the victim ends when police leave. In this case, victims' responses to the questions are interpreted or guessed by the officer, so the risk assessments can be completed. This is problematic for a risk assessment tool which is designed to provide a description of risk from the victims' perspective and in the victims' words. This practice raises questions about the accuracy of the information collected:

“Often they're not filled out at the scene, often they're filled out back here so some of the questions just aren't asked to the victim” (7).

This has particular implications for the Risk Assessment Questions for Adult Victims which are based solely on victim's statements:

"Sometimes it's hard to get the answers to the victims' questions, especially some of the ones who are constantly being beaten up and they just think it's their fault so they won't really cooperate with police” (8).

"Some people don't want to answer the questions. It can get a bit cagey when they still love them and all that and they don't really want to put them in hot water so there's not much you can do really" (30).

Along with officers involved in similar research (Humphreys et al, 2005), the constables in this study highlight that family violence offenders will often leave the scene before police arrive, making it difficult to gather the information required. It also is problematic for the risk assessment process when the parties involved will disclose their name and address only. In cases of non-violent verbal incidents, officers explain that apart from these basic details, parties involved are not legally required to provide further information and police cannot force them to do so: 
"A lot of cops will put a line through it and put not applicable because they haven't been given that information, and other than giving us their names they're not really obliged to" (26).

"What you get at the scene is what you're stuck with and most of the time all you've got is names and what not. Yeah it is quite hard to fill those silly things in sometimes, and I mean that's what I find the hardest thing” (5).

When victims do answer their questions, some officers are cynical about the truth of their responses. As such, these officers question the value of asking the risk assessment questions if the information collected is inaccurate:

"We're kind of depending on the information we get, like it's [the risk assessment] only as good as the information that's given to us by the victim, because that could not be accurate for a number of reasons" (9).

When victims are uncooperative, officers become frustrated as they feel they are wasting their time with little result when other emergencies require their response:

"I'm not trying to make out that we don't care, but sometimes you have to feel that there are other jobs going on and domestic violence is important but if the person isn't willing to tell you something?” (11)

In these situations, officers are uncertain about gathering the information they need if they cannot get it from the parties involved, and believe that the reliance on family violence victims as the primary source of information is problematic:

"You can ask as many questions as you want but if the person doesn't want to give you the information that they should, it is going to totally affect the risk score that you're going to get” (20). 
"If you sit down with the victim then obviously there's still a bunch of questions that they're not going to know about previous convictions and things like that or animal abuse or whatever the weird questions there are on it” (7).

Given the complex dynamics of family violence, it is common for there to be no clearly defined victim and offender, with both parties having engaged in some form of family violence behaviour. This comment was made most frequently in relation to the Risk and Lethality Assessment Worksheet, which is difficult to complete accurately in this situation. Klinger (1997) draws our attention to the fact that many victims of crime are often offenders as well. That is, citizens who are victimised in one instance are offenders in another. Similarly, officers in this study describe attending the same address on multiple occasions to find one party the victim one day and the offender on another:

"One minute we could be locking someone up and they will hate us but then we they need our help they'll have no qualms in calling us and saying oh I need your help now when they were the offender just the other day" (3).

In these cases, officers struggle to complete the risk assessments which are set up in a way that requires officers to identify characteristics of the victim and offender. Officers find the risk assessments difficult to complete because the risk markers are absent at minor incidents when physical violence has not occurred:

"For some of the minor ones or that aren't the typical victim/offender scenarios they're quite hard to do, so if you can't clearly identify one party then who do you ask the questions?” (13)

"I also have occasions where no one is the offender or they're both offenders, they've both given as good as they've got and how can you have two offenders, it doesn't allow you to do that" (11). 
In these cases, officers will either leave difficult questions unanswered which distorts the risk score produced, or rely upon their professional intuition to answer the questions:

It's quite big, it's not always done, it's not done at the scene, not always enough detail to fill the form in, so I think fairly useless” (11).

In their objection to completing risk assessments at the scene, officers draw upon the barriers presented by the context of their work and the particular dynamics of family violence to justify their failure to adhere to the policy. Some officers have found that by memorising the risk assessment tools the assessments can be completed without physically doing so at the scene. This is one of several strategies officers use to negotiate the policy while continuing to fulfil their reporting requirements:

"A lot of its memory, but obviously a good cop will have a good notebook, so you can always refer back and think, oh yeah. And you sort of get into the groove of it, when you do them you know what sort of questions to ask because you're familiar with them, so you can cover off the while FVIR while you're speaking with them" (3).

In this scenario, officers do not diverge from the policy because they do not support it but because they believe that environmental realities of their role are not conducive to doing so. Officers explain their resistance to completing risk assessments at the scene as based in part on victims reacting negatively to them doing so. These officers explain how it is impersonal and intrusive to complete a risk assessment in front of a victim and strongly believe that victims experience this as confrontational and intimidating:

"Pulling out the booklet...it feels like you're sitting there demanding an answer. It's confrontational” (6).

"I know that if they were attending my domestic it would seem so impersonal, here's this book, this question, this question. It's not 
personal. It's much better to establish rapport with them. Otherwise it seems like as you've done your book then you're going to bugger off and you don't really care, you care more about the paperwork than with them" (14).

They point out individual questions which provoke negative reactions from victims. Commonly, when the risk assessment questions about animal abuse are asked at minor verbal incidents, they are misinterpreted by victims who mistake police as more concerned with animals than people:

"When you're the cop on the ground asking these questions, you know, instances of animal abuse, it's not really relevant to the situation that you're dealing with there. But I can understand why perhaps people that do abuse animals are more possible to abuse people but trying to ask that in the situation, in the atmosphere that you're in, it doesn't always work" (1).

Officers feel that using the FVIR at the scene interrupts their ability to maintain both a conversation and eye contact with the victim. This presents police as unsympathetic as they prioritise paperwork over victim-care:

"Pulling out your book like this and reading it, it's just very straightforward and authoritative and just looks like you're pushing paper around and you're not empathising with what's going on in the situation” (1).

Officers feel that the FVIR is a visible barrier to effective communication and prevents them from establishing trust and rapport with victims. When a number of these factors co-exist, they interact to culminate in the belief held by a considerable number of officers that it is impractical to complete the risk assessments at the scene of a family violence event. They believe the characteristics of a typical family violence event make it difficult and unsuitable to complete paperwork there: 
“Quite often when you're at a job you don't go and actually sit at a table in the middle and have a conference or something...you might be standing in the middle of a hallway, a garden or anywhere, and you've got your little torch and you're having a look...I wouldn't imagine that I'd ever do one at the scene" (10).

Others find completing a report at a citizens' property an undesirable and difficult location, especially during the night or outside a house. These officers feel that there are too many complicating variables at the scene for paperwork to be completed there and favour completing documentation at the police station. Here, it is more stable and quiet than the scene, and officers can sit down to focus on the assessments:

"There is often too much going on at the scene and if there have been injuries sustained or the victim is hysterical then it is very difficult and impractical to complete the report at the scene according to the policy" (16).

"It's easier back at the station when you can sit down with a flat surface and no bumps and just go through it” (14).

These comments demonstrate how the practice of completing paperwork at the scene conflicts with officers' ideas about good police work and their priorities at the scene. In order to investigate family violence well, officers believe trust and rapport, sensitivity and understanding are vital and they are accustomed to doing this through their conversations with the public. From the comments officers made, they feel that the skills they usually associate with good police work are interrupted when they are required to use the FVIR booklet at the scene. There are important implications of this finding and as Edwards (2005) points out, rules which are seen as unnecessary, inappropriate or unfair by those to whom they apply are likely to be disregarded.

It is widely recognised that officers place the greatest importance on those skills of good police work developed through on-the-job experience (Reuss-Ianni, 
1983). Skills such as calming down situations, talking to parties and using the establishment of trust and rapport to ascertain the course of events are mastered through experience and distinguish professionalism. When explained in this way, officers' resistance to reporting at the scene is unsurprising. As Bayley and Bittner (1984) highlight, police officers are especially sensitive to acting in ways to avoid aggravating hostile situations. This explains why officers strongly believe that using their notebook to gather evidence is more acceptable to victims. They believe that a police officer's notebook is an ordinary part of routine police work, while the FVIR booklet is unknown and disconcerting:

"I don't think that most of the people that we deal with would like seeing something like that. I mean they're happy for you in those tense situations to write something in your notebook but if you pulled out a big flipping thing” (10).

Based on their comments documented in chapter three, constables in this study have little practical experience of completing risk assessments at the scene. While officers may have very little or no experience of using risk assessment at the scene of family violence events they most certainly have experience of frontline policing and responding to family violence within this environment. As will be discussed in greater detail by main discussion in chapter six, expressions of the street-level culture of police are problematic for police reformers. When officers engage in expressions of this culture, they serve to justify and reinforce a view of their role and their world which is counterproductive to certain forms of change.

It is argued that street-level police continue to define their primary role as crime fighters and law enforcers (Goldstein, 1979; Bittner, 1979; Sun, 2003), despite a growing body of research which reveals that responding to service calls and minor incidents make up the bulk of what they do, officers in this study continue to see their role along traditional lines (Bittner, 1979; Sun, 2003). Indeed, Reiner (1992) writes that frontline police believe their mission is to 'protect and serve'. While traditional notions of police work are not the only terms officers in this study use to describe frontline policing, these notions remain predominant for 
officers' role orientations as they speak of their responsibility to protect the public, maintain safety and enforce the law:

"Basically protect and serve the lovely citizens of Hamilton... enforcing the law and making sure everyone's not breaking it and if they do, basically bringing them to justice” (5).

Frontline officers recognise that proactive policing is a secondary duty which usually occurs during periods of decreased workloads or crime crackdowns. In line with international studies of street-level policing, proactive police work remains an ancillary task to the primary emergency-response function of GDB constables in New Zealand:

"Occasionally you get a bit of time to go and out and look for people doing things but generally it's busy just responding to 111 calls" (7).

"Some proactive stuff as well...if we've got nothing else to do we drive around and just keep an eye on the public" (25).

Officers highlight the service aspects of frontline policing and the significant role of pro-active activities:

"A lot of it isn't criminal stuff, a lot of it is kind of, to tell you the truth more social work now I've noticed. Proactive stuff, so like pulling over cars and just going around the streets at night to catch out any people who are about to commit crimes or stuff like that. Talking to people, just making them feel safe in the community" (4).

The comments officers make do suggest that they see themselves as employees of NZ Police who carry out 'real' police work, and some officers resist accepting a role which is not defined by fighting crime and law enforcement:

"Which in a way is making us act like counsellors type thing" (4). 
When officers were asked to describe the duties they commonly perform, responding to family violence features prominently. Instead of dismissing family violence from their mandate as has occurred in the past (Dobash \& Dobash, 1979; Edwards, 1989; Newbold \& Cross, 2008), police officers in this study see family violence as a significant and important part of frontline police work. This is an important finding, and one which refutes traditional examinations of the failure of police to adequately respond to family violence, as will be addressed in the following chapter. This finding did not appear to be related to officers' awareness of scope of the study, as the focus on the policing of family violence was not explained to officers' until after they had discussed the role of GDB constables.

This chapter has highlighted that officers believe the context of frontline policing and the particular dynamics of family violence are problematic for their implementation of risk assessment. There is evidence that these factors have a powerful influence on where, when and how the risk assessment process occurs. Earlier chapters demonstrated the equally powerful influence of police organisational culture on the implementation of risk assessment, as officers experience and interpret the challenges and realities of the risk assessment model in the frontline environment through the lens of their organisational street-cop culture. Similarly, the chapter highlighted how officers believe that victims' negative reactions to their use of the FVIR at the scene also prevent them from complying with the policy. Frontline officers hold two particular beliefs about the risk assessment process, namely that; it is inappropriate to complete risk assessments at the scene, and that it is impractical to complete risk assessments at the scene. However, it was found that officers have little experience of completing risk assessments there. Instead, these beliefs originate not from actual experience, but from cultural beliefs of 'real' police work and how it should be done. These beliefs serve to justify officers' continual departure from organisational policy. 


\section{Chapter 6}

\section{A discussion of the key findings}

The major objective of this thesis was to analyse the use of the New Zealand Police risk assessment tools by GDB constables, as part of the frontline response to family violence in the Waikato District. The research drew upon officers' accounts of their experiences of using risk assessment and how the process is being implemented six months after its introduction. In discussing these findings, the key notion of organisational culture provides the theoretical framework upon which they are explained. The influence of police culture on the successful implementation of the risk assessment model is examined and it is argued that this culture provides the point of reference through which officers view their work and changes to it. Officers' resistance to the bureaucratisation of frontline policing and the tension between the street-cop and management-cop cultures evident in the findings, form a major focus of the discussion also.

The significant majority of the constables in this study are positive about the introduction of family violence risk assessment. They recognise and support the need for police to improve their response to victims of family violence, especially in situations where there is an ongoing pattern of abuse and a risk of further serious violence. A small number of officers believe that they can already see benefits to their family violence investigations from the use of risk assessment. These officers believe that they investigate and report family violence situations with a greater level of accuracy. Officers feel that the risk assessment tools allow them to uncover cases of undisclosed abuse and the introduction of the risk assessment tools has raised their awareness of risk in family violence situations. These are very positive findings.

The research considered constables' knowledge and understanding of the risk assessment requirements to find that all officers are aware of and have used the risk assessment tools. While officers complete the risk assessments for both family violence incidents and offences, often they do so because compliance with this aspect of the policy is strictly enforced by police management. A very 
small number of officers wholly support the use of risk assessment for family violence offences. They believe that recording detailed information for all family violence situations and identifying risk markers is important. They recognise the value of assessing the level of risk and maintaining records of this information. Comments made by a majority of officers indicate that in the absence of enforcement, many would use the risk assessment tools for family violence offences, but not for incidents. Those officers who do not support the use of risk assessment for family violence incidents, question the relevance and necessity of assessing risk in these cases. They base this belief on the absence or ambiguity of risk markers at family violence incidents and believe that risk assessment is meaningless in this context. Instead, officers believe that the extent of risk assessment required should reflect the seriousness of the event. This belief is related to the importance that frontline police officers place on their discretion. Officers believe that they should be responsible for determining whether risk assessment is required, based on their evaluation of the situation. They argue that the attending officers, opposed to police management, are best placed to make this decision.

There are a number of other reasons why officers question the use of risk assessment at family violence incidents. Firstly, constables' lack of understanding of the purpose of risk assessment is problematic for the success of the risk assessment model. The majority of officers do not support the use of risk assessment for family violence incidents because they are unaware of the background and objectives of the risk assessment tools. It is largely unknown that the risk management strategy relies upon GDB constables to begin and maintain the risk assessment of violent families. Results from a recent study of police risk assessment conducted by Perez-Trujillo and Ross (2008) in Australia found that officers tended to see the possibility of future assault as unlikely or rare when attending a first incident of family violence. Perez-Trujillo and Ross (2008) concluded that police expect most incidents of family violence to be a one-off situation, as opposed to a pattern of abuse. In the current study, probationary constables especially, spoke of knowing very little of the dynamics and nuances of this complex crime. Perez-Trujillo and Ross' (2008) findings highlight that the success of risk assessment strategies rely upon officers' 
understanding that the information they collect is essential to identifying at-risk situations. Furthermore, Janet Chan (1995) also adds that officers will resist and ignore policies and directives from headquarters which they do not understand. The current study found strong evidence to suggest that the officers do not place the effort and emphasis they should on completing the risk assessments because they are unaware of the importance of their role in tracking and managing at-risk families. Thus, it is imperative that constables know that they play a vital role in the beginning of an ongoing process of risk management (Dutton \& Kropp, 2000).

In contravention of the policy, the significant majority of constables complete family violence risk assessments at the police station, largely at the end of the shift. It appears that the policy requirement that the assessments be completed at the scene of the event is not enforced. As a result, there is a discrepancy between the risk assessment policy developed by police management and how risk assessment is implemented in practice by frontline police in the Waikato District. Some officers are unaware of this requirement, while others consciously choose to ignore it. This pattern of use is informed by the failure of police management to enforce compliance with this aspect of the policy. This finding reiterates the crucial role of section supervisors for ensuring the support and compliance of frontline officers for the successful implementation of the model (Humphreys et al, 2005).

Instead of completing the assessments at the scene, frontline officers manoeuvre and negotiate the policy to reflect the situational realities of their work, and their perceptions of how risk should be assessed by frontline officers. This is an unsurprising finding as studies of police work have consistently demonstrated the number of ways street-level police circumvent and resist policies and procedures to which they do not subscribe (Manning, 1992; Davies \& Thomas, 2003; Rowe, 2007). The concept of organisational culture is useful for explaining how the maxims of the subculture of street-level police and the discretion afforded to their role are employed by frontline police officers to resist reforms imposed by the management-cop culture. 
Firstly, it is interesting that, despite having little or no experience of completing risk assessment at the scene, officers successfully justify their failure to complete risk assessment there. Officers draw on their 'working knowledge' as an experiential framework to explain the activities of frontline police officers (Skolnick, 2005). Specifically, they express culturally-informed beliefs about the nature of frontline policing and the importance of officer discretion, to argue that it is impractical and inappropriate for risk assessments to be completed at a family violence scene. While there was little empirical basis for these beliefs, they nonetheless have a powerful influence on the implementation of the risk assessment model. Officers have little experience of using risk assessment at the scene, but they justify their failure to do so by referring to the situational difficulties of dealing with family violence at the frontline. This finding highlights the predominance of frontline officers' perceived wisdom of policing and the cultural framework through which they view changes to their environment. Given the autonomy and isolation of much of their work, officers' justifications are accepted and reinforced by police management. A conflict between police management and the General Duties Branch can also be seen. The two cultures disagree on how risk assessment should be done, and the result of this is a discrepancy between decisions made at one level by police management and the actions taken by frontline police at another. While the risk assessments are being completed and the information is being gathered, it is not in the manner intended by police management or for which the risk assessment tools are designed.

Despite the fact that the officers in this study do not complete risk assessments at the scene of family violence events, according to officers' narratives they believe that the introduction of risk assessment has improved the investigation of family violence. A small number of officers strongly support the introduction of risk assessment. These officers understand the purpose and objectives of risk assessment, and are more likely to complete the risk assessment tools at the scene. Overall, most officers believe they have changed their approach to questioning at the scene; they are conscious of issues of risk and the potential for escalation, and engage in a greater level of investigative police work. These officers ask more questions and more specific questions about relationship 
history and risk, as they investigate beyond the current event alone. Their comments indicate that the introduction of risk assessment has established risk as a central concern in the frontline response to family violence.

Despite the positive improvements to the investigation and reporting of family violence, these changes are neither pervasive nor complete. In fact, some officers display a definite resistance to the risk assessment requirements and changing their investigative behaviours at family violence scenes. This study found three main reasons for the limited impact of risk assessment. Firstly, the failure to complete risk assessment at the scene means that issues around risk are considered retrospectively once officers have left the scene. As such, officers complete the assessments to satisfy their paperwork requirements and the information captured by the tools and the risk score generated do not influence their actions at the scene.

Secondly, officers' knowledge and understanding of the risk assessment strategy is limited. As previously highlighted, the officers are either unaware or unsure of the aims and objectives of risk assessment. In particular, the pivotal importance of the role of frontline police in the assessment of risk is largely unknown. Subsequently, officers do not place the level of importance and effort required on completing risk assessments. A lack of knowledge and understanding also generates difficulties with the risk assessment questions, as officers do not understand the answers required or the importance of particular questions to the assessment of risk. In turn, they believe that the risk assessment tools are restrictive and subjective, favouring their professional judgment of risk over that of the structured risk assessment tools. In turn, the validity and the accuracy of information collected are undermined, as is officers' support for the risk assessment model.

Finally, the context of frontline policing and the distinctive characteristics of family violence present a number of challenges for the risk assessment process. It was evident that context has a powerful influence on the risk assessment process. It is clear that the variables at any given scene shape officers' decisions about where, when and how the risk assessment process will occur. Irrespective 
of the policy, the research found that officers continue to decide where risk assessment should occur based on situational variables and culturally informed beliefs about 'real' police work. Factors such as officers' safety concerns, the availability of weapons and the influence of alcohol, influence officers' decisionmaking regarding the use of family violence risk assessment. However, the findings indicate that officers have little experience of completing the risk assessments at the scene. Therefore, it is officers' beliefs about the relevance of the risk assessment tools and their own criteria of street-level policing which drive decisions about the appropriate time and location to assess risk. These beliefs serve to justify a continual failure to do so. The officers' themselves explain their failure to adhere to the policy as the product of necessity. They firmly believe that the situational realities of frontline policing, and the unique characteristics of this crime are problematic for formally assessing risk at the scene. Whether or not this is the case, the isolated and unsupervised nature of frontline policing allows street-level police to successfully use the difficult situational realities of their work and the autonomy of their position to justify their actions to supervisors, physically removed from the frontline environment (Reiner, 1992; Reuss-Ianni, 1983). Thus, it is not necessarily the nature of the environment in which they work which is challenging for the risk assessment process, but the way in which these environmental conditions are viewed by officers through the values and norms of organisational culture. Indeed, as was identified by a small number of officers, difficult situational and environmental characteristics are features of a wide variety of tasks frontline police are required to do.

Traditional examinations of the police have held the misogynist and discretionary culture of police organisations responsible for their inadequate response to family violence (Edwards, 1989). Officers in this study do not believe that the risk assessments are irrelevant for the family violence events police attend and this finding challenges traditional feminist explanations of a sexist organisational culture dismissive of interpersonal violence. As such, these explanations of police culture cannot sufficiently explain the findings of this study (Chan, 1997; Chan, 2003). A more complex explanation is required which can account for the interaction between the characteristics of family violence and 
the frontline environment in which risk assessment occurs, amidst the pervasive influence of organisational culture. It is not argued that a focus on the misogynist aspects of police culture is incorrect, they most definitely contribute to explanations of the inadequate police response to family violence; but that there are other aspects of the occupational culture of street-level police which explain the discrepancy between policy and practice which traditional feminist examinations do not consider. Instead, findings from this study demonstrate that it is the interpretation of the characteristics of family violence, within the operational context of frontline policing, by officers through the police occupational culture which is problematic for the successful implementation of risk assessment. In the same way, Chan (1996) argues that police culture results from an interaction between the 'field' of policing and the various dimensions of organisational knowledge. An alternative explanation will now be presented.

A rejection of moves for change and reform by the rank and file is not new. Certainly, research on police reform has consistently provided examples of resistance from street-level police (Reuss-Ianni, 1983; Reiner, 1992; Barton, 2003; Paoline, 2003; Humphreys et al, 2005; Rowe, 2006; Chan, 2007; PerezTrujillo \& Ross, 2008). Undoubtedly, a major finding of this study is the powerful effect of police culture on the implementation of reform. Barton (2003) identifies that while rank and file officers recognise that there is a need for some reform, many reject calls for greater change. To illustrate the persistent nature of this culture, Savage (1991) describes police occupational culture as the "Berlin wall of policing” (Savage, 1991, as cited in Barton, 2003, p.3). In finding that frontline police officers in New Zealand do not comply with the policy, this research reaches a typical conclusion (Humphreys et al, 2005). Indeed, Barton (2003) argues that the failure of reformers to fully understand the street-cop culture has undermined the successful implementation of numerous police reforms. Accordingly, a consideration of the key elements of the street-cop culture will explain why change in the police response to family violence is difficult to achieve (Barton, 2003). Studies of the implementation of similar risk assessment models in other jurisdictions and the reactions of street-level police to reform contribute to our understanding of the research findings (Humphreys et al, 2005; Bevir \& Krupicka, 2007). 
As evidenced in other studies of family violence risk assessment (Humphreys et al, 2005), officers in this study resist management's attempts to modify their role. The introduction of a compliance-based structured decision-making tool challenges the sanctity of frontline officers' discretion, autonomy and professionalism. This reform conflicts with officers' sub-cultural conception of frontline policing and the challenges to it that will be tolerated. In their abandonment of organisational rules and regulations, the street-cop culture provides the reference through which street-level police 'make sense' of change to their environment. Instead of following management directives, what officers do is framed more by their own criteria of police work than by the formal rules and regulations of the organisation (Reuss-Ianni, 1983; Weick et al, 2005; Chan, 2007). Most commonly, officers will interpret reforms to better fit with their experiences when there is a conflict between the reform and the local culture (Bevir \& Krupicka, 2007).

In terms of the risk assessment model, frontline officers believe that as professional crime fighters, they are the best placed to decide where a risk assessment is completed and for which cases. As already established, frontline officers in this study do not resist the risk assessment policy because of disbelief in the significance of family violence or a belief that traditional police responses to it are satisfactory. Indeed, officers cited responding to family violence as an important and prominent part of their role. As such, their resistance to the policy is an expression of the tension between the street-cop and management-cop cultures of police. While the two cultures of policing abstractly share the goals of combating crime and maintaining safety, the divergence lies in their beliefs about what 'real' police work is about and how it is achieved (Reuss-Ianni, 1983; Davies \& Thomas, 2003). As a result, street-cops show little commitment and support for management initiatives (Reuss-Ianni, 1983). This is unsurprising as a cynical view of police management is identified as a central tenet of the streetcop culture (Chan, 2003). Consequently, the limited buy-in management has secured from the General Duties Branch for the risk assessment model, is a consistent theme. Humphreys et al (2005) highlight the importance of risk assessment being 'sold' to frontline officers. They argue that this is crucial for officers to be committed to intensifying the investigative efforts that risk 
assessment requires. Buzawa and Buzawa (1996) highlight that police are in the frontline to prevent domestic violence. Thus, securing the commitment and support of these officers is essential for the success of risk assessment strategies.

As we have seen, officers resist management's attempts to redefine their role and in particular their attempts to do this with a compliance-based bureaucratic model. Due to their lack of understanding of the aims of risk assessment and the importance of their contribution to the risk assessment strategy, officers believe the risk assessment tools are another bureaucratic requirement that frontline police officers have been given to do for other branches of police and their interagency partners. Officers do not feel a sense of ownership in the process. Barton (2003) highlights that police officers are more likely to support initiatives when they feel they are central to the process and can see the benefits of their contribution. He quotes Fielding (1988) who comments:

"No reform can hope to succeed that does not enlist the support of the ordinary constables who construct the reality of the policing experience" (Fielding, 1988, as cited in Barton, 2003, p.3).

As such, it is important that police management promote to officers the benefits of completing risk assessment at the scene for limiting duplication of paperwork; ensuring accuracy and completeness of reports, and the benefits to the overall quality of family violence investigations. Police management must endorse the benefits of the FVIR and risk assessment for GDB constables, as opposed to benefits for organisation as a whole. At present, many officers believe that they complete risk assessments for the benefit of management rather than to their own advantage.

Another important theme was the prominent role of discretion in the risk assessment process. In the absence of a clear understanding of the requirements of the risk assessment model, officers draw on their professional intuition to assess risk. When the risk assessments are not completed at the scene and victims are not asked the relevant questions, officers rely upon their interpretations of the situation to complete the assessments. The failure to 
implement and use family violence risk assessment tools for the purpose and the way in which they were designed has a number of implications for the success of risk assessment. Most importantly, this impacts on the accuracy and reliability of the data obtained. Kropp (2004) argues that there is nothing more dangerous than a risk assessment based on inadequate information. He warns that inaccurate risk assessments can lead to an underestimation of risk. This will misinform victims, police units, and the support-agencies who rely on the information gathered by risk assessment. Furthermore, Reuss-Ianni (1983) adds that the structural and behavioural problems which plague officers and supervisors at the operational level of policing can undermine even the most sophisticated initiatives.

There are particular implications for the Victims Questions (B1) when officers fail to complete this risk assessment at the scene. Invariably, this pattern of use means that victims will not be asked the questions required. If this is the case, then the answers recorded are of little use to the FVT and serve only to distract from the true reality of the situation. The accuracy and value of victims' testimonies to the assessment of risk has been proven (Websdale, 2000; Goodman et al, 2000; Weisz et al, 2000; Heckert \& Gondolf, 2004; PerezTrujillo \& Ross, 2008), and this is a necessary aspect of the NZ Police risk assessment model. If officers were to comply with the policy they would gather more accurate information and more accurately predict the risk of future serious harm or murder. However, for this to occur, the meaning of particular questions must be clarified, so that officers no longer complete the risk assessment tools with professional intuition. As such, the final section of this chapter considers whether police management can address these issues by pursuing a compliancebased or professional-ethical approach to structuring the use of officer discretion.

Officers resist and resent the erosion of their discretion because of the increase in bureaucracy that it necessarily involves. Attempts to erode officer discretion have arisen out of studies demonstrating the inappropriate use of discretion by police officers, particularly in relation to the under and over-enforcement of particular crimes (Rowe, 2002), and the concern that police officers can display great bias in their enforcement of the law (Barton, 2003). Criticisms of police organisations as racist, sexist and prejudiced have naturally arisen from such 
findings. In an attempt to control discrepancies in the application of the law, police management has placed increasing emphasis on administrative rulemaking (Barton, 2003). This was a prominent theme in the study as officers' narratives demonstrate the high value they place on their discretion and their resentment towards changes in their work which erode it. From their research of the use of risk assessment by Police in Victoria, Australia, Perez-Trujillo \& Ross (2008) highlight that police officers can be reluctant to use paper-based forms to guide their judgment.

More often than not, measures to simultaneously curb the inappropriate use of officer discretion and hold officers to account for their actions has resulted in a trend toward the increasing bureaucratisation of frontline policing (Flanagan, 2008). Reuss-Ianni (1983) explains how the police management culture has bureaucratised police work in order to make it more easily managed. She argues that street-cops view bureaucratic controls as confusing, believing that they compound the difficulties they experience, rather than assisting them in dealing with the ambiguities and uncertainties of their role. Unlike crime fighting and law enforcement, it has been shown that paperwork is peripheral to police officers' role orientation and is seen as a distraction from the real 'mission' of frontline policing (Reiner, 1992; Ericson \& Haggerty, 1997; Barton, 2003; Campbell, 2004a; Chan, 2007). Paperwork is viewed with cynicism, as another attempt to curtail the discretion of frontline officers by using the 'paper trail' to ensure compliance and accountability (Barton, 2003).

As highlighted earlier, frontline police officers are able to exercise great discretion in their work, and this allows them to subvert or obstruct policing reforms initiated by police management (Reiner, 1992). Barton (2003) argues that frontline officers use their discretionary powers to successfully negotiate even the most specific legislation. Carbonatto (1998) similarly concluded that NZ Police did not always act in accordance with the domestic violence pro-arrest policy. Thus, measures to remove the discretion of frontline police officers are inextricably linked to issues of individual and organisational accountability, and the last three decades have seen a trend towards the increasing bureaucratisation of the public sector (Flanagan, 2008). With the rise of new discourses such as 
New Public Management across the public sector, management levels have increasingly bureaucratised the work of those below them (McLaughlin et al, 2001; Davies \& Thomas, 2003). Police services have not been exempt from this change (Flanagan, 2008). In an important review of the British Police Service, Flanagan (2008) identifies that there is a constant focus on the accountability of the public sector, and this has resulted in the unprecedented scrutiny and review of contemporary police services. In this review, Flanagan (2008) highlighted how these changes have increasingly bureaucratised police work and the past twenty five years have seen the paperwork officers are required to complete more than double. Thus, officers are not mistaken when they protest about the increase in paperwork required by their role.

Similarly, Janet Chan (2007) highlights how accountability in policing has become synonymous with paperwork. Bayley and Bittner (1984) also identify that accountability for their actions is a constant source of concern for police officers, who are continually reminded of the consequences of their behaviour. In such an environment 'risk avoidance' becomes orientated internally to the workplace. A recent longitudinal study by Chan (2007) found that while the need for police officers to be accountable was not challenged, officers frequently linked accountability with management's excessive concern with reporting. Chatterton (1989) refers to paperwork as 'defensive work', which acts as a mechanism through which the police 'cover their backs' (Campbell, 2004a). Van Mannen (2005) highlighted a similar pre-occupation with accountability among officers in his research. The current study saw officers connect an increase in family violence paperwork required with efforts to improve the accountability of police to victims of family violence. In the same way, officers in Humphreys et al's (2005) study drew links between the increased paperwork requirements of risk assessment and police accountability also. The risk assessment tools as well as the policy, seek to achieve discretionary decision-making. Some view bureaucratically imposed limits on behaviour as a way to limit bias in police decision-making. Conversely, others believe that compliance-based reforms detract from professional and ethical decision-making (Schneider et al, 1996). 
As such, the key findings in this study raise a number of important questions for both researchers and reformers, including whether the nature of police culture is conducive to reform and if the adoption of a compliance-based or a professionalethical model can best change the behaviour of police officers who endorse the street-cop culture. Those who argue that police organisational culture is static and inflexible believe that police will continue to negotiate and circumvent policy and there is little police management can do to avoid it (Paoline, 2003). Whilst police culture is most definitely a common and persistent barrier to reform, it is not impenetrable (Chan, 1996). To argue otherwise is to ignore that police culture exhibits continuous change, as the organisation itself faces greater public and legislative accountability (Paoline, 2003; Marks \& Singh, 2007). Janet Chan (1996) denies that police are insulated from attempts to achieve cultural change and she argues instead that police culture presents opportunities for change as well as resistance. From her research into police organisational culture and the socialisation of recruits, she concludes that police culture is less homogenous and much more open to change than was previously assumed (Chan, 1997). Specifically, Davies and Thomas (2003) argue that there has been significant change to the masculinities entrenched within the organisational culture of police and other research has highlighted the complexity of culture and variation among officers also (Paoline, 2003).

Change to the organisational culture of police in New Zealand can most strongly be seen in the dramatic transformation of the police response to family violence in recent decades (Chrichton-Hill, 2004; Newbold \& Cross, 2008). Similarly, the constables who participated in this study did not display the misogynist and prejudiced beliefs by which the police response to family violence was previously characterised. This research demonstrates that officers' attitudes and behaviour can be changed, as some officers in this study absolutely support the introduction of family violence risk assessment tools, and there was evidence of an improvement to officers' family violence investigations. Indeed, as police organisations become more demographically diverse with the inclusion of persons previously excluded from the organisation, the strength of a singular homogenous culture is diminished (Paoline, 2003). The conclusion reached by this study is that police can successfully achieve reform in this area if issues with 
the organisational sub-culture are dealt with, particularly issues around the use of officer discretion.

This leads to the question of whether a compliance-based or professional-ethical model of policing would best facilitate the implementation of the family violence risk assessment model. In recent years there have been attempts to enhance the status of police work by elevating policing to a profession. Moves to 'professionalise' the police have taken place at a time when the legitimacy and expertise of traditional police forces is increasingly challenged (Davies \& Thomas, 2003; Punch, 2007). Findings of police corruption, misconduct and malpractice by government commissions, the proliferation of specialist policing agencies and the recognition of plural and third-party policing, has seen increasing debate about the roles and responsibilities of the police (Chan, 2003; Stenning \& Shearing, 2005; Mazerolle \& Ransley, 2005).

Proponents of the professional-ethical model argue that street level police should be empowered through relevant training strategies to make better rather than less decisions (Davis, 1996). Police leaders have sought to achieve this with the inclusion of ethics and discretion as key topics in training programs and the creation of ethical and good-practice standards for officers (Chan, 2003). Similarly, a drive to enhance officers' educational qualifications has been seen as a recurring answer to the poor image of police (Punch, 2007). With his early discussion of police discretion, Herman Goldstein (1963) noted that one of marks of a true professional is the need for value judgments and for exercising discretion based upon professional competence. He concluded that to deny that police officers exercise discretion is to argue their role is simple and does not require judgment, and is thus unworthy of professional status. Officers themselves judge performance by the standard of the professional cop. They believe that professionalism is learnt through the on-the-job experience which allows them to recognise the people and situations which require their intervention (Reuss-Ianni, 1983; Van Maanen, 2005).

In support of the position taken by Goldstein (1963), Davis (1996) affirms that if we want police to use their discretion and their past experiences of operational 
policing to make situationally appropriate decisions, then we must move away from the traditional militarist command and control structure of police organisations. Davis (1996) highlights how an ethical-professional model recognises that police work is more than a straight application of rules, and in recognising this, police must be afforded professional status. However, an approach which empowers officers to act with discretion does not mean that they are left without the boundaries of principles and professional standards (Davis, 1996).

The implementation of family violence risk assessment by frontline police might be less discretionary if officers were empowered with the necessary knowledge of the model, its objectives and their role within it. Greater compliance with the policy may result and a need to further erode officers' discretion would then be unnecessary. Indeed, police training is one of the most direct ways available to foster police professionalism (Chan, 2003), and the findings discussed throughout this thesis have demonstrated areas where further training is required. However, Chan (2003) draws upon available evidence which suggests that no matter how enlightened training programs are intended to be, once recruits come face to face with the realities of operational police work they fall under the negative influence of the street-cop culture. She concludes that the operational police culture tends to undermine formal training and professionalism (Chan, 2003). Indeed, from officers' comments about the risk assessment approach, it is clear that they favour a professional-ethical model, compared with the compliance-based approach to risk assessment introduced by police management.

Alternatively, the use of administrative rule making approaches has gained considerable support from policy makers and police managers as a way to structure police discretion (Goldstein, 1979; Barton, 2003). Proponents of a compliance-based model warn that occupational professionalisation inevitably leads to increased autonomy and ultimately increased power for members of the organisation. They argue that professionalism could widen the police mandate and increase the opportunities for police to act with discretion. From this perspective, it is not professional police but accountable police that are required 
(Van Maanen, 2005). Findings from this study demonstrate that police management can ensure minimum reporting requirements with the use of compulsory information fields on police forms. Bayley and Bittner (1984) identify that such forms structure choice, because officers know that they will be held accountable for any departure from official policy. However they caution that such a model may encourage false reporting. For the NZ Police risk assessment model to be successful, officers must complete the risk assessments for all family violence events attended, and this must be done at the scene. Based on the research findings, a compliance-based approach could achieve this.

However, there will be occasions in which officer discretion is both required and desired in the police response to family violence and the assessment of risk. In support of this point, Davis (1996) argues that the advantages of professional judgment are not gained by completely controlling officers' decisions. In recognising that family violence situations are diverse, ambiguous and unpredictable, perhaps there must be greater room in the assessment tools for officers to include their professional judgement. From officers' comments about the risk assessment tools, no matter how clear the questions are, it is likely that some interpretation will be necessary in certain situations, in practice this means that discretion and the use of professional intuition is inevitable in the risk assessment process.

Whether a compliance-based model or a professional-ethical model is followed, the success of family violence risk assessment requires a change in police organisational culture. Specifically, those aspects of the street-cop culture that see frontline officers negotiate and resist the risk assessment policy. Barton (2003) warns that until police management recognise the importance of culture, they will continue to face barriers to reform and little change can be achieved. A need for such change was earlier recognised by Reuss-Ianni (1983) who highlighted that behaviour change requires a change in attitude to support a reform once it is introduced. She argues that changes in attitudes are essential for new principles and practices to be incorporated within the organisational culture of those charged with their implementation. Given that officers tend to continue to see their role as that of crime fighter and law enforcer, training should 
emphasise to officers the investigative benefits of completing risk assessment properly, such as the likelihood of successful prosecutions.

Despite improvements in the investigation of family violence, there is much work to be done to address early issues with the implementation of family violence risk assessment and to realise the full benefits of the model. Continuous improvement must be sought. From this study, the challenge for police is to finish translating the change in thinking which has occurred for most constables into a change in behaviour. It is clear that a number of the implications of the findings of this study affect the management level of police. While it is the street-cop culture which is problematic for reform, in order to address the subcultural problems the management-cop culture must involve and utilise section supervisors more widely in the risk assessment process. Supervisors must be actively involved in the risk assessment process and act proactively to consistently enforce the risk assessment requirements.

Police management must ensure that family violence risk assessment is implemented by GDB constables at the scene of family violence events. As previously indicated, officers need to be empowered with a greater understanding of the risk assessment tools and the risk assessment process. Furthermore, enforcing the use of risk assessment at the scene will involve obtaining greater support from section supervisors to support the goals and intentions of police management (Humphreys et al, 2005). Research has demonstrated that supervisors inform both the behaviour and attitudes acceptable under their command (Reuss-Ianni, 1983; Humphreys et al, 2005). Reuss-Ianni (1983) found that frontline supervisors were usually reluctant to make decisions and absolute rules that will set a fixed pattern of enforcement, and frontline officers are aware of this. In their evaluation, Humphreys et al (2005) advised that the risk assessment model would not be used appropriately if senior officers did not champion its introduction and ongoing use.

Given that much learning with the police occurs on-the-job, it is important that senior officers pass on their wisdom of risk assessment correctly to other constables. In addition, commitment and support from officers must be obtained, 
or as it has been shown, officers will continue to negotiate and resist those policies to which they do not subscribe (Reuss-Ianni, 19833; Humphreys et al, 2005), as they ignore and resist rules which they neither support nor agree with (Rowe, 2007). Feedback from street-level police should also be sought, and Bevir and Krupicka (2007) argue that initiatives which fail to consider the experience of the rank-and-file are likely to be limited. Their experiences could play an important role in the development of best practice guidelines and policies for the implementation of the risk assessment model. This practice would further assist in securing commitment and support from officers as management is seen to consider the realities of street-level policing (Barton, 2003).

\subsection{Concluding comments}

Two crucial overarching questions arise from these research findings. Firstly, whether the risk assessment initiative is being implemented as intended by police management? Chapters three, four and five have examined this issue in detail and the overall conclusion reached is that that it is not, as the study found considerable evidence of a discrepancy between management policy and operational practice. It is asked whether this discrepancy results from problems with the implementation of the risk assessment model, or as a consequence of issues related to the risk assessment tools themselves. Officers' comments indicate that aspects of both the implementation of risk assessment and the risk assessment tools generate difficulties for officers. In the absence of empirical confirmation of the validity of the tool (Websdale, 2000; Kropp, 2004; Humphreys et al, 20045), the answer to this question is unclear. While there is evidence of some validity of the Danger Assessment Scale for predicting domestic homicide (Weisz et al, 2000; Roehl \& Guertin, 2000), upon which one of the NZ Police risk assessment tools is based, but further research is essential (Weisz et al, 2000).

Secondly, it is asked whether the NZ Police family violence risk assessment model can improve the police response to this crime, if it is implemented as intended by police management. Based on these preliminary findings of a small study of GDB constables in areas of the Waikato District, if the model was 
implemented correctly, family violence assessment would have a greater impact on the family violence investigations of frontline police officers than is currently seen. The positive improvements made thus far indicate that change is possible. However, additional change to the assessment of risk for family violence events in a number of areas is required. These include; changes to the subculture of street-level police, improvements in officers' knowledge and support for the development, and changes in the management and training which accompany the NZ Police risk assessment model.

A broader, but related issue is whether police and frontline police particularly, are best placed to prevent serious and lethal family violence. Like crime generally, family violence is an established feature of our society which no amount of policing is likely to eradicate (Newbold \& Cross, 2008). Indeed, given that victims of family violence only come to police attention when contact is initiated by a witness or the victims themselves, the challenge of assessing and managing the risk of all family violence situations may be an impossible one. Given the time restraints and the reactive environment of most police responses to family violence situations, there may be limited opportunities for frontline police to assess risk as desired. A better strategy for the reduction and prevention of family violence may lie in responding to the causes, as opposed to the symptoms of this crime. Few believe or have ever argued that police or the criminal justice system alone can respond adequately to family violence (Radford \& Gill, 2006). Indeed, the community and other agencies have an important role to play in the prevention and reduction of family violence (Watt, 2008).

Research into risk assessment, particularly that used by police, remains in its infancy (Websdale, 2000; Kropp, 2004). There is an immediate need for the evaluation of both the implementation and the validity of family violence risk assessment tools. Kropp (2004) emphasises that not only must the validity and accuracy of structured risk assessments for predicting serious and lethal violence be proven, but it is important that any evaluations examine the actual workings of risk assessment models as well. Perez-Trujillo and Ross (2008) identify the importance of understanding the realities of risk-related decision-making so that 
these realities can be factored into the design of risk assessment instruments. Thus, both the process and the outcomes of risk assessment tools require immediate attention (Kropp, 2004; Humphreys et al, 2005). Furthermore, the assessment of risk by frontline police officers is the first stage in the process of risk management (Dutton \& Kropp, 2000; Kropp, 2004; Humphreys et al, 2005) and what police and their partner agencies do with the information collected by constables should be included in the focus of future research also (Humphreys et al, 2005). Furthermore, as the landscape of policing continues to change both internationally and in New Zealand, a more detailed and nuanced view of police organisational culture is required, which can account for the changing role and composition of the public police (Marks \& Singh, 2007).

\subsection{Overall conclusions}

To reiterate those points made in chapter two, the research findings cannot be said to reflect the experiences and practices of frontline police officers in New Zealand. Given the small sample size, the findings can only be said to provide an early insight into the experiences and opinions of a group of constables from the Waikato District General Duties Branch who regularly attend family violence occurrences and use police family violence risk tools. The implementation of the NZ Police risk assessment model is at an early stage and it is inevitable that challenges will be encountered as frontline officers and police management adjust to the changes it involves. The impending policy should clarify some of the issues highlighted by this study.

It is important to recognise that the research has also identified a number of positive changes to the investigation of family violence the model has achieved. Particular aspects of the risk assessment tools are especially appreciated by officers and they believe that the introduction of risk assessment has enhanced the family investigations they conduct. Officers in this study support any development in the police response to this crime, and for this reason, the introduction of risk assessment in the Waikato District should be viewed with optimism. 
This study has demonstrated that an understanding of the features of the environment in which frontline policing occurs and the affect of police organisational culture on the success of reform extends our understanding of the barriers contemporary police organisations continue to face. The research has demonstrated the influence of contextual factors on the implementation of family violence risk assessment. Equally, it has examined how frontline officers continue to draw upon maxims of organisational knowledge to implement risk assessment, in the face of the confusion that this initiative presents and its conflict with their cultural beliefs about 'real' frontline police work. It is clear that this study is only the beginning of the research and evaluation of the NZ Police family violence risk assessment model necessary for the future development and success of this exciting initiative. 


\section{References}

Abrams, M. L., Belknap, J., \& Melton, H. C. (2001). When domestic violence kills: The formation and findings of the Denver Metro Domestic Violence Fatality Review Committee. Denver: Project Safeguard.

Barton, H. (2003). Understanding occupational (sub) culture - a precursor for reform. International Journal of Public Sector Management, 16(5), 346358.

Barwick, H., Gray, A., \& Macky, R. (1996). The Domestic Violence Act 1995: Process Evaluation. Wellington: Ministry of Justice, Department of Courts.

Bayley, D. H., \& Bittner, E. (1984). Learning the skills of policing. Law \& Contemporary Problems, 36(4), 35-59.

Bayley, D. H. (2005). What do the police do? In Newburn, T. (Ed.), Policing: Key readings (pp. 141-149). Portland: Willan Publishing.

Bazley, M.C. (2007). Report of the Commission of Inquiry into Police Conduct. Retrieved March 25, 2008 from http://www.cipc.govt.nz/cipc. nsf/wpg_URL/Agency-Commission-of-Inquiry-into-Police-ConductReport-of-the-Commission-of-Inquiry-into-PoliceConduct?Open Document.

Belfrage, H. (2008). Police-based structured spousal violence risk assessment: the process of developing a police version of the SARA. In Baldry, A.C., Winkle, F.W (Ed.), Intimate Partner Violence Prevention and Intervention (pp. 33-44). New York: Nova Science Publishers Inc.

Bevir, M., \& Krupicka, B. (2007). Police reform, governance, and democracy. In O’Neill, M., Marks, M., \& Singh, A.M. (Ed.), Police Occupational Culture: New Debates and Directions (pp. 153-180). Oxford: Elsevier JAI. 
Bittner, E. (1979). The Functions of the Police in Modern Society. Cambridge: Oelgeschlager, Gunn \& Hain.

Bittner, E. (2005). Florence Nightingale in pursuit of Willie Sutton: A theory of police. In Newburn, T. (Ed.), Policing: Key readings (pp. 150-172). Portland: Willan Publishing.

Busch, R., Robertson, N. (2000). Innovative approaches to child custody and Domestic Violence in New Zealand. Journal of Aggression, Maltreatment and Trauma, 3(1), 269-299.

Butler, E.K., Winifree, T., \& Newbold, G. (2003). Policing and gender: Male and female perspectives among members of the New Zealand police. Police Quarterly, 6(3), 298-329.

Buzawa, E.S., \& Buzawa, C.G. (1996). Domestic Violence: The Criminal Justice Response. Newbury Park: Sage Publications.

Campbell, J. (1995). Assessing Dangerousness: Violence by Sexual Offenders, Batterers and Child Abusers. Newbury Park: Sage Publications.

Campbell, E. (2004a). Police Narrativity in the Risk Society. British Journal of Criminology, 44(5), 695-714.

Campbell, J. (1986). Nursing assessment for risk of homicide with battered women. Advances in Nursing Science, 8(4), 36-51.

Campbell, J., Webster, D., Koziol-McLain, J., Block, C. et al (2003). Risk factors for femicide in abusive relationships: Results from a multi-site case control study. American Journal of Public Health, 93(7), 1089-1097.

Campbell, J. C. (2004b). Helping women understand their risk in situations of intimate partner violence. Journal of Interpersonal Violence, 19(12), 1464-1477.

Carbonatto, H. M. T. (1998). The Criminal Justice System Response to Domestic Violence in New Zealand. A thesis submitted to the Victoria University of 
Wellington in fulfilment of the requirements for the degree of Doctor of Philosophy in Criminology.

Chan, J. (1996). Changing police culture. British Journal of Criminology, 36(1), 109-134.

Chan, J. (2003). Fair cop: Learning the art of policing. Toronto: University of Toronto Press.

Chan, J. (2007). Making Sense of Police Reforms. Theoretical Criminology, 11(3), 323-345.

Chrichton-Hill, Y. (2004). Violence in interpersonal relationships. In Connolly, M. (Ed.), Violence in Society: New Zealand Perspectives (pp. 65-80). Christchurch: Te Awatea Press.

Cross, J. (2006). Policing Family Violence in Christchurch. A thesis submitted to the Canterbury University in fulfilment of the requirements for the degree of Master of Arts in Sociology.

Davies, A., \& Thomas, R. (2003). Talking cop: Discourses of change and policing identities. Public Administration, 81(4), 681-699.

Davis, M., Ed. (1996). Police, Discretion, and Professions. In Kleinig, J. (Ed.), Handled with Discretion: Ethical Issues in Police Decision Making (pp. 13-35). United States: Lanham, Rowman \& Littlefield Publishers.

Dobash, R. E., \& Dobash, E. (1979). Violence against wives: A case against the patriarchy. New York: Free Press.

Dutton, D. G., \& Kropp, P.R. (2004). A review of domestic violence risk instruments. Trauma Violence Abuse, 1(2), 171-181.

Edwards, S. (1989). Policing 'domestic' violence: Women, the law and the state. London: Sage Publications. 
Edwards, C. (2005). Changing Policing Theories for $21^{\text {st }}$ Century Societies, $\left(2^{\text {nd }}\right.$ Ed.). Sydney: The Federation Press.

Ericson, R. V. (1982). Reproducing order: A study of police patrol work. Toronto: University of Toronto Press.

Ericson, R.V., \& Haggerty, K.D. (1997). Policing the Risk Society. Oxford: Clarendon Press.

Family Violence Coordination Unit. (2007). Family violence risk assessment and risk management. Melbourne: Family Violence Coordination Unit, Department for Victorian Communities.

Felson, R. B., Ackerman, J.M., \& Gallagher, C.A. (2005). Police intervention and the repeat of domestic assault. Criminology, 43(3), 563-588.

Flanagan, R. (2008). The review of policing: Final report. Retrieved March 5, 2009, from http://police.homeoffice.gov.uk/publications/police-reform /Review of policing final report/.

Goldstein (1979). Improving policing: A problem-oriented approach. Crime Delinquency, 25, 236-258.

Goldstein, H. (1963). Police discretion: The ideal versus the real. Public Administrative Review, 23(3), 140-148.

Goodman, L., Dutton, M.A., \& Bennett, L. (2000). Predicting repeat abuse among arrested batterers: Use of the Danger Assessment Scale in the Criminal Justice System. Journal of Interpersonal Violence, 15(1), 63-74.

Gregg, L. (2007). Collaboration in family violence intervention: A process evaluation of the Hamilton Family Safety Team. A thesis submitted to the Waikato University in fulfilment of the requirements for the degree of Master of Social Sciences.

Gregory, J., \& Lees, S. (1999). Policing Sexual Assault. London: Routledge. 
Hanmer, J., Griffiths, S., \& Jerwood, D. (1999). Arresting evidence: Domestic violence and repeat victimisation. London: Home Office, Policing and Reducing Crime Unit.

Hassan, N. (2007). Measuring the effectiveness of police handling of prosecution cases in the specialist Family Violence Court. A thesis submitted to the Victoria University of Wellington in partial fulfilment of the requirements for the degree of a Masters of Strategic Studies.

Heckert, A. D., \& Gondolf, E.W. (2004). Battered women's perceptions of risk versus risk factors and instruments in predicting repeat re-assault. Journal of Interpersonal Violence, 19(7), 778-800.

Hilton, Z. N., Harris, G.T., \& Rice, M.E. (2001). Predicting violence by serious wife assaulters. Journal of Interpersonal Violence, 16(5), 408-423.

Hirschel, J. D., Dean, C.W., \& Lumb, R.C. (1994). The relative contribution of domestic violence to assault and injury of police officers. Justice Quarterly, 11(1), 99-117.

Holdaway, S. (1994). Recruitment, race and the police subculture. In Stephens, S., \& Becker, S. (Ed.), Police Force, Police Service: Care and Control in Britain (pp. 67-84). London: MacMillan Press Ltd.

Hoyle, C., \& Sanders, A. (2000). Police response to domestic violence: from victim choice to victim empowerment? The British Journal of Criminology, 40(1), 14-36.

Hoyle, C. (2008). Will she be safe? A critical analysis of risk assessment in domestic violence cases. Children and Youth Services Review, 30, 323337.

Humphreys, C., Thiara, R.K., Regan, L., Lovett, J., Kennedy, L., \& Gibson, A. (2005). Prevention not Prediction? A preliminary evaluation of the Metropolitan Police Domestic Violence Risk Assessment Model 
$\left(\right.$ SPECSS $\left.^{+}\right)$. London: Centre for the Study of Safety and Wellbeing, University of Warwick and Child and Woman Abuse Studies Unit.

Hyman, P. (2000). Women in the CIB: Opportunities for and barriers to the recruitment, progress and retention of women in the criminal investigation branch. Wellington: New Zealand Police.

Klinger, D. A. (1997). Negotiating order in patrol work: An ecological theory of police response to deviance. Criminology, 35(2), 277-306.

Kropp, P. R., \& Hart, S.D. (2004). The development of the brief spousal assault form for the evaluation of risk (B-Safer): a tool for criminal justice professionals. Ontario: Department of Justice Canada.

Kropp, P. R. (2004). Some questions regarding spousal assault risk assessment. Violence Against Women, 10(6), 676-697.

Laing, L. (2004). Risk Assessment in Domestic Violence. Retrieved March 31, 2008 from http://www.austdvclearinghouse.unsw.edu.au/topics/topics pdf files/risk assessment.pdf.

Lerman, L. (1992). The decontextualisation of domestic violence. The Journal of Criminal Law and Criminology, 83(1), 217-240.

Lievore, D., \& Mayhew, P. (2007). The scale and nature of family violence in New Zealand: A review and evaluation of knowledge. Wellington: Ministry of Social Development.

Manning, P.K. (1992). Information technologies and the police. Crime and Justice, 15, 349-198.

Marks, M., \& Singh, A.M. (2007). Conclusion: Taking stock and looking ahead in police cultural studies. In O’Neill, M., Marks, M., \& Singh, A.M. (Ed.), Police Occupational Culture: New Debates and Directions (pp. 349-367). Oxford: Elsevier JAI. 
Martin, J., Langley, J., \& Millicamp, J. (2006). Domestic violence as witnessed by New Zealand children. The New Zealand Medical Journal, 119(1228), $1-14$.

Mazerolle, L., \& Ransley, J. (2005). Third party policing. Cambridge: Cambridge University Press.

McLaughlin, E., Muncie, J., \& Hughes, G. (2001). The permanent revolution: New labour, new public management and the modernisation of Criminal Justice. Criminal Justice, 1(3), 301-318.

Morris, A., \& Reilly, J. (2003). New Zealand National Survey of Crime Victims 2001. Wellington: Ministry of Justice, Crime Prevention and Criminal Justice Group.

Mossman, E., Mayhew, P., Rowe, M., \& Jordan, J. (2008). Literature review about the barriers to recruiting a diverse police workforce. Wellington: Crime and Justice Research Centre, Victoria University of Wellington.

Newbold, G., \& Cross, J. (2008). Domestic violence and pro-arrest policy. Social Policy Journal of New Zealand, 33, 1-14.

New Zealand Police. (2008a). [Women killed by an intimate partner in the context of family violence]. Unpublished raw data, available on request from the National Statistics Team, National Police Headquarters.

New Zealand Police. (2008b). Beat and Patrol Constables. Retrieved August 9, 2008, from http://www.police.govt.nz/service/beatandpatrol.

New Zealand Police. (2008c). Community Policing. Retrieved December 3, 2008, from http://www.police.govt.nz/service/community.

New Zealand Police. (2008d). Statement of Intent 2008/09-2010/11. Wellington: New Zealand Police.

New Zealand Police. (2008e). Waikato District - New Zealand Police. Retrieved August 9, 2008, from http://www.police.govt.nz/district/waikato. 
New Zealand Police. (2009). [Recorded occurrences of family violence in the Waikato District]. Unpublished raw data, available on request from the National Statistics Team, National Police Headquarters.

Paoline, E. A. (2003). Taking stock: Toward a richer understanding of police culture. Journal of Criminal Justice, 31, 199-214.

Pence, E. (1983). The Duluth Domestic Abuse Intervention Project. Hamline Law Review, 6, 247-275.

Perez Trujillo, M., \& Ross, S. (2008). Police response to domestic violence: Making decisions about risk and risk management. Journal of Interpersonal Violence, 23(4), 454-473.

Punch, M. (2007). Cops with honours: University education and police culture. In O’Neill, M., Marks, M., \& Singh, A.M. (Ed.), Police Occupational Culture: New Debates and Directions (pp. 105-128). Oxford: Elsevier JAI.

Radford, L., \& Gill, A. (2006). Losing the plot? Researching community safety partnership work against domestic violence. The Howard Journal of Criminal Justice, 45(4), 369-387.

Reiner, R. (1992). The Politics of the Police. Toronto: University of Toronto Press.

Reuss-Ianni, E. (1983). Two cultures of policing: Street cops and management cops. New Brunswick: Transaction Books.

Reuss-Ianni, E., \& Ianni, F.A.J. (2005). Street cops and management cops: the two cultures of policing. In Newburn, T. (Ed.), Policing: Key readings (pp. 297-314). Portland: Willan Publishing.

Roehl, J., \& Guertin, K. (2001). Intimate partner violence: The current use of risk assessments in sentencing offenders. The Justice System Journal, 21(2), 171-198. 
Rosenbaum, D.P. (2006). The limits of hot spots policing. In Weisburd, A., \& Braga, A. (Ed.), Police innovation: Contrasting perspectives. Cambridge: Cambridge University Press.

Rowe, M. (2002). Policing diversity: Themes and concerns from the recent British experience. Police Quarterly, 5(4), 424-426.

Rowe, M. (2007). Rendering visible the invisible: Police discretion, professionalism and decision-making. Policing and Society, 17(3), 279294.

Russell, M., \& Light, L. (2006). Police and victim perspectives on empowerment of domestic violence victims. Police Quarterly, 9(4), 375-396.

Saunders, D. G. (1995). Prediction of Wife Assault. In Campbell, J.C. (Ed.), Assessing Dangerousness: Violence by sexual offenders, batterers and child abusers (pp. 68-95). London: Sage Publications.

Schneider, A. L., Ervin, L., \& Snyder-Joy, Z. (1996). Further exploration of the flight from discretion: The role of risk/need instruments in probation supervision decisions. Journal of Criminal Justice, 24(2), 109-121.

Sherman, L. W., \& Berk, R.A. (1984). The specific deterrent effects of arrest for domestic assault. American Sociological Review, 49(2), 261-272.

Skolnick, J. H (2005). A sketch of the policeman's 'working personality'. In Newburn, T. (Ed.), Policing: Key readings (pp. 264-279). Portland: Willan Publishing.

Stenning, P.C., \& Shearing, C.D. (2005). Reforming police: Opportunities, drivers and challenges. The Australian and New Zealand Journal of Criminology, 38(2), 167-180.

Stephens, B.J., \& Sinden, P.G. (2000). Victims' voices: Domestic assault victims' perceptions of police demeanour. Journal of Interpersonal Violence, 15(5), 534-547. 
Sun, I. Y. (2003). Police officers' attitudes toward their role and work: A comparison of black and white officers. American Journal of Criminal Justice, 28(1), 89-108.

Van der Heyden, J. (1997). Opportunities for New Zealand Police to change organisational culture. The GSBSM Project Report Series 18. A thesis submitted for the partial fulfilment of MBA in Business and Government Management in the University of Victoria, Wellington.

Van Maanen, J. (1975). Police socialisation: A longitudinal examination of job attitudes in an urban police department. Administrative Science Quarterly, 20(2), 207-228.

Van Maanen, J. (2005). The asshole. In Newburn, T (Ed.), Policing: Key readings (pp. 280-296). Portland: Willan Publishing.

Waddington, P. A. J. (1999). Police (canteen) sub-culture: An appreciation. British Journal of Criminology, 39(2), 287-309.

Walby, S., \& Allen, J. (2004). Domestic violence, sexual assault and stalking: Findings from the British Crime Survey. Retrieved October March 31, 2009, from http://www.familieslink.co.uk/download/jan07/HORS\%20276.pdf.

Walton-Moss, B. J., Manganello, J., Frye, V., \& Campbell, J.C. (2005). Risk factors for intimate partner violence and associated injury among urban women. Journal of Community Health, 30(5), 377-389.

Watt, K. (2008). Understanding risk factors for intimate partner femicide: The role of Domestic Violence Fatality Review Teams. In Baldry, A.C., \& Winkle, F.W (Ed.), Intimate partner violence prevention and intervention (pp. 45-60). New York: Nova Science Publishers Inc.

Websdale, N. (2000). Lethality assessment tools: A critical analysis. VAW Net, 17. Retrieved March 20, 2008 from National Electronic Network on Violence Against Women. 
Weick, K. E., Sutcliffe, K.M., \& Obstfeld, D. (2005). Organising and the process of sensemaking. Organisation Science, 16(4), 409-421.

Weisz, A. N., Tolman, R.M., \& Saunders, D.G. (2000). Assessing the risk of severe domestic violence: The importance of survivor's predictions. Journal of Interpersonal Violence, 15(1), 75-90.

Wilson, M., \& Daly, M. (1993). Spousal homicide risk and estrangement. Violence and Victims, 8(1), 3-16. 


\section{Appendix A Information Sheet}

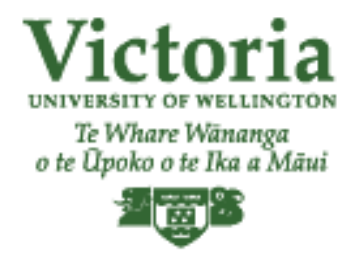

\section{INFORMATION SHEET}

\section{Research into New Zealand Police Officers Use of Risk Assessment in Family Violence Cases}

The purpose of this research is to provide an insight into the experience of front line officers implementing the family violence risk assessment model in the Waikato District. This research is being conducted by Stephanie Grant, under the supervision of Victoria University of Wellington, as part of a research project for a Master of Arts in Criminology.

It is hoped that valuable information will be collected on the issues and difficulties that officers face using the risk assessment tools, as well as provide an opportunity for officers to make suggestions for improvement on the risk assessment tools and how they are used.

Interviews will be conducted in the Waikato area during mid-September 2008 and it is anticipated that interviews will take around one hour to complete. A consent form will be provided for you to sign at the interview if you agree to be involved. Consent can be withdrawn without reason at any time up until $\underline{1}^{\text {st }}$ November 2008 when the data analysis will commence, by contacting Stephanie Grant via email or phone, as provided below.

Comments made in the interviews will remain confidential. Interview tapes and notes will be seen only by me and my supervisors and will be stored securely and confidentially at Victoria University. All interview tapes and notes will be destroyed at Victoria University on completion of the marking and moderation process. Names will not be used in any research reports and nothing will be published that will identify individual research participants. Where individual quotes are reported in the thesis, attention will be given to ensure no information is reported that will identify you.

This is an independent study conducted under the supervision of Victoria University and all data collected will be used solely for the completion of a Masters thesis. If any other use is undertaken your consent will be sought separately for this. You may stop the interview at any time and decline to answer individual questions without giving a reason for doing so. 
As with all research conducted by Victoria University involving human subjects, ethical approval has been given for this research by the Victoria University Ethics Committee. From NZ Police the Waikato District Commander and National Managers have given their approval for the research to be conducted.

You will have an opportunity to access a summary of the findings at the end of the research and the final Masters Thesis will be able from Police National Headquarters and the Police College Library.

For any further information do not hesitate to contact myself or my supervisors. Your comments and experiences are vital to this piece of research and to the improvement of the NZ Police risk assessment model. Your participation is greatly appreciated.

Kind regards,

Stephanie Grant

Primary researcher

Stephanie Grant

0275830067/04 9707935

grantstep@student.vuw.ac.nz
Research supervisor

Dr Michael Rowe

044639452

Michael.Rowe@vuw.ac.nz 


\title{
Appendix B Consent Form
}

\author{
Victoria \\ UNIVERSITY OF WELLINGTON \\ Te Whare Wānanga \\ o te Qpoko o te lka a Mäui

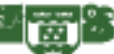

INTERVIEW CONSENT FORM

\section{Research into New Zealand Police Officers Use of Risk Assessment in Family Violence Cases}

Name:

Contact telephone number:

I have been provided with an information sheet which explains the purpose of the research, and my right not to answer any questions or to stop the interview, without having to explain why.

I agree to participate in this interview on the understanding that what I say will be kept confidential by the researcher and will only be used for research purposes. My name will not be used in any research reports and nothing will be published that will identify me.

I understand that I can withdraw any information I provide, without any reason up until $1^{\text {st }}$ November 2008 when the data analysis will commence, and I have been provided with the details to do this. I agree that I have had an opportunity to have any questions answered in full prior to the interview.

I agree to the interview being tape recorded and understand that the tape will be destroyed at Victoria University on completion of the marking and moderation process.
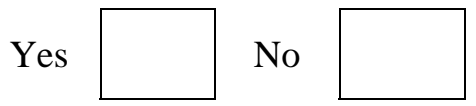

I want to be provided with a summary of the research findings.

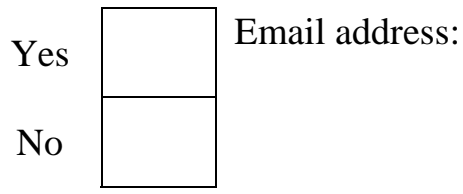

Signed:

Date: / 2008 


\section{Appendix C Interview Schedule}

Note: The italicised comments above each of the questions sections are my notes only, based on particular things I wanted to find out from my research questions, rather than

questions to be asked during the interviews.

\section{Role Orientation}

What are front line officers' understandings of frontline policing?

Looking at front line officers' perceptions of their role in the wider scheme of police and within NZ Police

1. What does your job involve? In 1 to 2 sentences tell me about your job within NZ Police. (Prompt: types of calls, types of responses, time of day, number of officers, where - patrol car or police station, experience of officers attending)

a. What do you do on a day to day basis?

b. What shapes the jobs you deal with on your shift? (Sergeant, calls from public, set own agenda etc)

c. Compared to other branches of NZ Police, how do you think General Duties Branch Policing differs from Road Policing, CIB, or Community Policing?

d. What makes your General Duties Branch policing distinctive?

e. How would you compare this to other areas of police work not at the front line?

What are front line officers' perceptions of their role in policing family violence? To what extent are front line officers aware of and using the risk assessment tools?

2. What is your experience of dealing with family violence as part of your job?

a. How frequently do you attend family violence incidents?

b. What is it like? What are some particular characteristics of dealing with family violence incidents? (Prompt: Complex, difficult, time consuming, rewarding, violent, stressful, straight forward, similarity/patterns between cases etc)

(Angry, violent, weapons, hostile, hard to handle)

i. Kinds of jobs? 

ii. Significant issues?
iii. Special skills you need for family violence? (Patience, firm, strong, empathetic etc)

3. Do you approach family violence differently to other types of crimes? What do you do in terms of family violence being a distinctive type of crime? (Extra caution to avoid potential violence, non-confrontational, park car around corner, work in pairs etc)

a. Get them to describe the procedure (mention risk assessment unprompted)

\section{Awareness and Use}

To what extent are front line officers aware of and using the risk assessment tools?

4. Are you familiar with the risk assessment process (as part of the POLFVIR)? What are the three aspects/key components of the risk assessment process? (Red flags, lethality questions, 3x victim's questions) To see if officers can identify the individual components.

a. Get officers to talk about the individual aspects. Explain:

i. Have you used them? All 3?

ii. How much? Why?

iii. When do you use it? Types of incidents NOT location.

iv. When do you not use it? Types of incidents NOT location.

v. Are they compulsory? (What is the policy/guidelines around use)

5. What is the purpose of carrying out risk assessments?

6. How frequently do you use risk assessment at a family violence incident? (Probe for each component of the risk assessment model)
a. Red flags
b. Lethality questions
c. 3x victims' questions

i. Why do you think this is so?

7. When is it carried out? (LOCATION: Scene, patrol car, station, other?)

a. How user-friendly is it? (Probe: complicated/straightforward, easy to follow/unguided?) 
b. How do you feel about using it?

8. Do you know what happens to the information collected by this process?

\section{Behavioural change}

9. Has introduction of the risk assessment process changed how you deal with family violence? Note:: This won't apply to those who were not in the job prior to introduction of the risk assessment process.

10. How? (Positive, negative, neither; individual change, collective change). (Probe: any change in behaviour, attitudes, perceptions, practice). (If not: Already doing this work, already know this from experience, not always appropriate or necessary, officers should retain discretion etc) - Includes those not in the job prior to introduction of the risk assessment process.

a. Improves/inhibits investigations?

b. Improves/inhibits service provided to victims?

c. Formalised/standardised response?

d. Consistency of response?

e. Improved attention and effort?

f. Affects on efficiency at the scene?

g. Quality of evidence gathered?

\section{Opinions of risk assessment}

What is the tension between management policy and operational practice?

11. Is the model helpful to you in dealing with family violence incidents? (Prompt: Guidelines, expectations, procedure, less discretion (good/bad?), more paperwork, no change in what we already did, already know this from experience, undermines discretion, unnecessary, doesn't get done, unclear, lengthy, complicated)

12. How useful do you find the following risk assessment sections of the POLFVIR? (Probe: positive, negative, helpful, inhibitory, favourite tool) - Probe for each component of the risk assessment model
a. Red flags
b. Lethality questions
c. 3x victims' questions

13. In general, how do you find using the following the risk assessment sections of the POLFVIR?

a. Do you feel confident completing the risk assessment sections? 
b. Ease of use: time consuming, time available, paper work levels affected etc

14. Are there any things that prevent you from completing risk assessments? (Clarify: at all, effectively) (Prompt: training, support, supervision, workload). Including, any ways the tool could be improved? (Prompt: Forms streamlined, explanations on forms, compulsory fields etc)

a. What changes would allow you to complete risk assessments more effectively?

b. What changes would make completing risk assessments easier?

\section{Training and support}

15. How would you describe the risk assessment training?
a. What did it involve?
b. How long did the training session last?
c. How long ago did you receive training?
d. Now having used the risk assessment sections, is there anything you feel should have been included in the initial training?
e. Will you receive further training on risk assessment?
f. After you completed the training, did you discuss it with your supervisor?

16. If no training was received - why was this?

17. Did this training provide you with the necessary skills? (Note: apply only to those who were trained). Why, why not?

18. How applicable are the risk assessment tools to most family violence incidents you attend?

19. How would you describe support and supervision from your supervisor?
a. Are your risk assessments checked?
b. Are your risk assessments followed up on?
c. How would you go about getting assistance/second opinion with completing a risk assessment if you needed it?

\section{Further comments}

20. Do you have any other comments on what we have discussed today?

21. Are there any areas that we have not discussed which you think are important? 


\section{Appendix D Analysis Matrix}

The framework provided below illustrates the key themes and sub-themes which were used to conduct the qualitative thematic analysis for this thesis. There are six key headings in this analysis matrix, which each reflect a section in the interview schedule. Below these headings, the sub-themes which arose from the data have been listed. A description of the methods of analysis is provided in chapter 2, section 2.3 .

\section{Role Orientation}

\begin{tabular}{|l}
\hline Frontline policing \\
\hline First line of response/out on the street \\
Respond to calls for service from the public \\
Emergency crisis/reactive \\
Traditional crime fighting conception of police work \\
General duties \\
Proactive \\
Pressure from police management
\end{tabular}

Frontline policing is distinct from the work of other NZ Police branches Initial police response

Ability to react to any possible situation/more intense and dangerous

Wide range of duties versus specialist tasks of other units

Limited involvement at the incidents attended

Reactive versus proactive response (immediate response versus planned approach)

General characteristics of family violence events as experienced by GDB constables

Dangerous, safety concerns prominent (safety of officers, victims and children), availability of weapons and common presence of intoxicated persons

Entering the private domain, intimidating for police, police abuse

Emotional nature of family violence, victims distressed or uncooperative, impacts of a victim with a history of severe and repeat victimisation

The characteristics of individual incidents are unknown until police arrive

Family violence includes a wide range of behaviours which occur along a continuum of seriousness

Characteristics of GDB constables who respond to family violence well

On-the-job experience

Important life experience

Communication and interpersonal skills

Empathetic and understanding

Ability to calm a situation down and take control

\section{Awareness and Use}

Awareness of family violence paperwork requirements

Awareness of purpose

Awareness of the policy 
Confusion with the policy

No knowledge of the policy

Labour intensive and time consuming

It is a positive change that the risk assessment tools are included in the FVIR

Context of risk assessment use

Police car

Police station

Scene of the family violence event

Explanation for the location of reporting

\section{Behavioural Change}

No behaviour change

No behaviour change only a change in recording practices

Change in thinking about family violence and risk at the scene

Ask more questions

Ask more specific and different questions

Investigations are more structured

Investigate more thorough and obtain more information

Benefits for accountability

\section{Opinions of Risk Assessment}

Relevance of risk assessment to family violence events

Different risk assessments should be used for verbal non-violent events

Risk assessment is relevant to all family violence events

Risk assessment is irrelevant to family violence events

Risk assessment is relevant for family violence offences only

Difficulties experienced with the risk assessment tools

High number of risk assessments to complete during a shift due to high number of family violence events attended

Time restraints associated with the pressures and high workload of frontline policing

Sensitive and inappropriate questions which are impersonal and traumatic for victims

Questions are unclear

The answers required on the risk assessments are unclear

The information required is unavailable

Difficulties experienced using the risk assessment as a result of context

Rely on victim cooperation

Safety concerns mean officers are reluctant to complete risk assessments at the scene

Victims react negatively to officers completing reports at the scene

Using the FVIR disrupts rapport and appears insensitive

\section{Auditing Process}

Awareness of sergeant's role

Awareness of the role of the FVT and/or the FST

Confusion about the auditing process

No knowledge of the auditing process 
Negative perceptions of the auditing process

Importance of auditing process for accountability

Issues around officer discretion

Tension between management-cop and street-cop culture

\section{Training}

Received training but dissatisfied with this training

Received training and satisfied with this training

Did not receive training

No training initially but received training at a later date

Unsure if they received training

Received training but did not believe this was help 
Appendix E Family Violence Investigation

Report 